\title{
Thermal Analysis of Refrigeration Systems Used for Vaccine Storage:
}

Report on Pharmaceutical Grade Refrigerator and Household Refrigerator/Freezer

Michal Chojnacky

Wyatt Miller Gregory Strouse 
NISTIR 7753

\title{
Thermal Analysis of Refrigeration Systems Used for Vaccine Storage:
}

\author{
Report on Pharmaceutical Grade Refrigerator \\ and Household Refrigerator/Freezer
}

\author{
Michal Chojnacky \\ Wyatt Miller \\ Gregory Strouse \\ $P M L$
}

September 2010

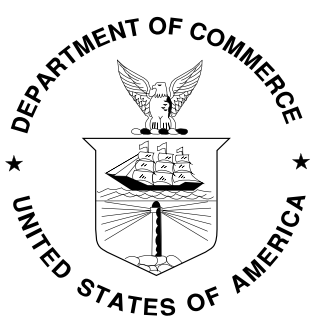

U.S. Department of Commerce

Gary Locke, Secretary

National Institute of Standards and Technology

Patrick D. Gallagher, Director 


\begin{abstract}
Each year, billions of dollars of vaccines are stored in refrigerators at the facilities of a variety of medical providers. Many vaccines must be maintained in the range $2{ }^{\circ} \mathrm{C}$ to $8{ }^{\circ} \mathrm{C}$ to retain product potency. We have tested the performance of two types of refrigerators to determine if these refrigerators are suitable to this task, and to identify proper storage and temperature monitoring methods. Nineteen calibrated Type T thermocouples, distributed through the refrigerator interior, served as reference thermometers. Attachment of thermocouples directly to vaccine vials gave accurate measurements of the vaccine temperature, which often differed from the air or interior wall temperatures during door openings or defrost cycles. A household type dual-zone refrigerator/freezer [refrigerator compartment capacity $=0.400 \mathrm{~m}^{3}(14.13 \mathrm{cu}$. ft.)] and a pharmaceutical grade refrigerator [capacity $=0.489 \mathrm{~m}^{3}$ ] proved fully adequate at maintaining vial temperatures within the desired $2{ }^{\circ} \mathrm{C}$ to $8{ }^{\circ} \mathrm{C}$ range, independent of how the refrigerator was loaded. Tests of intermittent and continuous door opening and of simulated power outages demonstrated the value of adding water bottles to the door as a thermal ballast in the dual-zone refrigerator model. We tested five electronic data loggers as a means of continuously logging refrigerator temperatures. Properly located, data loggers and temperature monitors accurately monitored vial temperatures for extended periods.
\end{abstract}

\title{
1. INTRODUCTION
}

Each year, millions of dollars worth of vaccines are lost in the United States due to improper storage conditions. In order to maintain potency, many common vaccines must be kept refrigerated between $2{ }^{\circ} \mathrm{C}$ to $8{ }^{\circ} \mathrm{C}$. When vaccines are exposed to temperatures above or below this range, their potency is reduced until they eventually become completely ineffective.

A number of factors influence whether vaccines stored in a refrigerator remain within the prescribed temperature range. Vaccine storage trays or boxes can obstruct refrigerator air flow, creating areas pockets of colder or warmer air. Vaccines stored in certain locations, such as near the refrigerator cooling unit, may be kept significantly colder than if placed in the main body of the refrigerator. Some factors may vary drastically between different refrigerator types (e.g. dorm style, freezerless, dual zone or pharmaceutical grade). Parameters such as temperature control stability, air circulation patterns, defrost cycles, and long-term drift of the temperature set point can play a major role in determining whether a refrigerator maintains suitable vaccine storage conditions. Because of this, simply setting a refrigerator to a temperature between $2{ }^{\circ} \mathrm{C}$ to $8{ }^{\circ} \mathrm{C}$ may not actually result in stored vaccines being kept within that temperature range.

Accurate monitoring of vaccine temperatures requires attention to the type of monitoring system used and its installation in the refrigerator. Different types of temperature monitoring systems can influence both the accuracy of the temperature measurement and the ease of use. Depending on the placement of a temperature monitoring device, as well 
as the time or frequency that its readings are recorded, the resulting temperature readings may or may not correspond to actual vaccine temperatures.

In this study, we explored a number of these factors in an attempt to determine which have the greatest impact on vaccine temperature. From this, we can make experimentally verifiable recommendations regarding the best and worst vaccine storage configurations and temperature monitoring setups. We used an array of calibrated thermocouples, mounted in multiple locations, as reference thermometers. An automated data-acquisition system measured the thermocouple temperatures continuously, for a wide variety of operating conditions. Attaching thermocouples directly to vaccine vials gave temperature measurements highly indicative of the actual vaccine temperatures.

We report measurements for two refrigerator styles: a) "dual-zone" household refrigerator/freezer unit [total capacity $=0.515 \mathrm{~m}^{3}(18.2 \mathrm{cu} . \mathrm{ft}$.)], and b) a pharmaceutical grade refrigerator [capacity $=0.489 \mathrm{~m}^{3}$ ]. The pharmaceutical refrigerator demonstrated excellent temperature stability. However, both models are fully adequate for refrigerated vaccine storage, and with a few simple precautions both can successfully maintain appropriate storage temperatures under varying conditions.

In this investigation, we repeated the procedures used in our earlier evaluation of a dormitory-style refrigerator and a freezerless household refrigerator used for vaccine storage, as reported in NIST-IR 7656 [1]. Another study exploring the suitability and performance of pharmaceutical grade refrigerators for vaccine storage was conducted by the Test Research Group for the Australian General Practice Network [2]. For further information on vaccine storage practices, including official storage guidelines from the Centers for Disease Control, consult references 3 - 6.

\section{EXPERIMENTAL METHOD}

Prior to this study, members of the NIST Thermometry Group visited seven Vaccines for Children (VFC) clinics in order to observe their vaccine storage practices. In our experimental trials, we attempted to replicate the range of vaccine storage conditions witnessed at VFC clinics.

This range included variations in loading density. For example, during influenza season, a large influx of patients could leave a clinic with dwindling vaccine supplies. At this time, their storage refrigerator would probably contain a low packing density. Other times, such as immediately following a large vaccine shipment, the same storage refrigerator might be filled to maximum storage capacity with boxes of vaccines. This would be considered a high density load. By testing a variety of low, medium, and high packing densities, we were able to quantify how this type of normal inventory fluctuation affects refrigerator performance.

We also experimented with different packaging styles. Many of the clinics surveyed used plastic trays or bins to organize and store their vaccine inventory. We also observed 
vaccines stored inside cardboard shipping boxes, as well as in their original light cardboard packaging. The Centers for Disease Control and Prevention (CDC) guidelines advise storing vaccines in plastic trays rather than uncontained on shelves or inside shipping boxes. However, during very busy times, clinic workers might temporarily store vaccines in their shipping containers until they can be organized into appropriate bins. As a result, we investigated whether these different packaging methods would affect the refrigerator's ability to maintain the proper vaccine storage temperatures. In this study, we performed trials using three different packing styles: 1) vaccines stored exclusively in plastic trays; 2) vaccines stored inside various boxes, such as cardboard shipping boxes or their original thin cardboard packaging; and 3) vaccines stored in a mixture of plastic trays and cardboard boxes.

In our survey of VFC clinics, we noted multiple locations storing water bottles in refrigerator doors or in the main space of the refrigerator, a practice recommended by the Centers for Disease Control. This is because the additional thermal mass of the water bottles should act as a temperature ballast, lessening the impact of potential temperature fluctuation sources. To test the effectiveness of this practice, we performed some trials with water bottles filling the refrigerator door, and some trials without. The volume of water bottles used was equal to $4 \%$ of the total refrigerator capacity.

We performed a cycle of measurements on two different refrigerators (a full-size, dualzone refrigerator/freezer unit and a full-size, pharmaceutical grade model) using permutations of the variables described above. Prior to the first cycle of measurements, the refrigerators were adjusted to give a storage temperature between $4{ }^{\circ} \mathrm{C}$ and $5{ }^{\circ} \mathrm{C}$. A complete listing of the different trials and order in which they were performed is contained in the measurement matrix (Table 1) shown below. In our testing of the dualzone refrigerator model, we performed trials both with and without water bottles in place. We did not use water bottles in any of our trials of the pharmaceutical refrigerator, because the model we tested featured a sliding glass door rather than a solid door with storage compartments. After completing the trials with varying load densities, packing styles, and with/without water bottles, we performed some additional trials designed to simulate some of the different conditions that a VFC clinic might experience. In this study, our focus was primarily limited to storage practices for refrigerated vaccines. Because of this, while completing the matrix measurements using the dual-zone model, we only considered the refrigerator chamber of the unit. The freezer control settings were left unchanged throughout the course of measurement collection, and the freezer cavity was left empty. In practice, provider offices may use both the freezer and refrigerator compartments of a single unit to store refrigerated and frozen vaccine simultaneously. Several VFC program coordinators and on-site assessors have expressed concerns that adjusting freezer temperature controls might impact storage temperature inside the refrigerator cavity, possibly damaging vaccines. To address this issue, we performed a short case study examining whether varying freezer temperature has a resulting impact on refrigerator compartment conditions. Our method and results from this study are described in Section 3.1.10. 
During the first cycle of trials, the refrigerator door was kept closed. However, in every day use, it is not possible to keep a refrigerator door closed at all times. The door must be opened briefly in order to add and remove vaccines for use. To mimic the opening and closing of the refrigerator door that occurs with normal use, we performed trials in which the door was opened at 15 minute intervals for 15 seconds at a time, over the course of at least $2 \mathrm{~h}$. We carried out these measurement using at least two different packing schemes for each refrigerator. We also recorded data with the refrigerator door left ajar for approximately $1 \mathrm{~h}$, in order to observe what occurs if a clinic employee forgets to completely close the door.

Another non-idealized but common experience at VFC clinics is fluctuation in the storage room temperature. Poor HVAC control, inclement or seasonal weather changes, and planned or unplanned outages can all cause unexpected room temperature fluctuations. We wished to determine whether these types of changes affect a refrigerator's ability to maintain the required $2{ }^{\circ} \mathrm{C}$ to $8{ }^{\circ} \mathrm{C}$ temperature range. To do this, we recorded refrigerator temperature data while increasing the ambient room temperature by several degrees. As before, these trials were repeated for each refrigerator using at least two different packing schemes.

In the final set of trials, we observed what occurs to refrigerated vaccine stores in the event of a power outage. In these trials, the refrigerator was set up to run normally. After starting the measurement collection system, the refrigerator was unplugged and data collection continued overnight.

Table 1. Matrix of measurements performed for each refrigerator.

\begin{tabular}{|c|c|c|c|c|c|c|c|c|}
\hline \multirow[t]{2}{*}{ Trial } & \multicolumn{3}{|c|}{ Load Density } & \multicolumn{3}{|c|}{ Packing Style } & \multirow{2}{*}{$\begin{array}{c}\text { Water Bottles } \\
\text { (dual-zone trials only) }\end{array}$} & \multirow[t]{2}{*}{ Measurement Parameters } \\
\hline & Low & Medium & High & Trays & Boxes & Mixed & & \\
\hline 1 & $x$ & & & $\bar{x}$ & & & & normal \\
\hline 2 & & $x$ & & $x$ & & & & normal \\
\hline 3 & & $\mathrm{x}$ & & $\mathrm{x}$ & & & $\mathrm{x}$ & normal \\
\hline 4 & & $x$ & & & $x$ & & $x$ & normal \\
\hline 5 & & & $\mathrm{x}$ & & $\mathrm{x}$ & & $\mathrm{x}$ & normal \\
\hline 6 & $x$ & & & & & $x$ & $x$ & normal \\
\hline 7 & & $\mathrm{x}$ & & & & $\mathrm{x}$ & $\mathrm{x}$ & normal \\
\hline 8 & & & $\mathrm{x}$ & & & $\mathrm{x}$ & $\mathrm{x}$ & normal \\
\hline 9 & & & $x$ & & & $x$ & $\mathrm{x}$ & increase room temp \\
\hline 10 & & & $x$ & & & $\mathrm{x}$ & $\mathrm{x}$ & periodic door opening \\
\hline 11 & & & $x$ & & & $x$ & $x$ & power off \\
\hline 12 & & $x$ & & & & $\mathrm{x}$ & & normal \\
\hline 13 & & $x$ & & & & $\mathrm{x}$ & & increase room temp \\
\hline 14 & & $x$ & & & & $\mathrm{x}$ & & periodic door opening \\
\hline 15 & & $x$ & & & & $x$ & & power off \\
\hline
\end{tabular}

To determine the temperature distribution throughout the entire refrigerator space, we arranged 19 Teflon-insulated, Type T thermocouples in various regions in the refrigerator body. The thermocouples were attached to refrigerator walls or storage trays, hung in air, mounted inside of glycol-filled bottles, or most importantly, taped to actual vaccine vials. The most important feature of a refrigerator used for vaccine storage is its ability to maintain a vial temperature between $2{ }^{\circ} \mathrm{C}$ to $8{ }^{\circ} \mathrm{C}$. Therefore, monitoring actual vial 
temperature in addition to other locations inside the refrigerator enabled us to effectively evaluate each refrigerator's performance. The installation pattern also allowed us to determine how well thermometers placed in different areas match the actual vaccine temperatures. This helped us produce useful guidelines for the best placement of refrigerator temperature monitoring devices.

The thermocouple reference junctions were maintained at $23{ }^{\circ} \mathrm{C} \pm 0.002{ }^{\circ} \mathrm{C}$ in an auxiliary stirred water bath. Prior to use, we calibrated each thermocouple at the melting point of ice $\left(0{ }^{\circ} \mathrm{C}\right)$ by placing the thermocouple measuring junction in a Dewar flask containing crushed ice and distilled water. The flask itself rested inside the refrigerator under study. An automated, $6 \frac{1}{2} 2$ digit voltmeter monitored the voltage produced by each thermocouple.

In addition to the 19 thermocouples used to monitor temperature, we tested several different electronic temperature loggers throughout the course of this study. At present, VFC clinic workers are required to check a thermometer placed inside the refrigerator at certain intervals each day. Barring any inaccuracies inherent to the device or poor placement of the thermometer, this type of monitoring is still likely to be insufficient. Refrigerator temperature is not constant over time, so a simple thermometer check once or twice a day will not give an accurate indication of whether the vaccines have remained within the designated temperature range.

Inexpensive electronic data loggers (e.g. self-contained temperature recording devices) may provide a useful alternative by giving 24-hour-a-day monitoring of refrigerator temperature. To investigate their utility, we positioned six different data loggers inside each refrigerator throughout the study in order to compare their performance to that of the thermocouples. Placement of the thermocouples and data loggers was kept consistent for the duration of trials in each refrigerator. The thermometer installation patterns for each refrigerator are shown in Figs. 1 and 2 below.

Table 2 summarizes the equipment used for this study. 


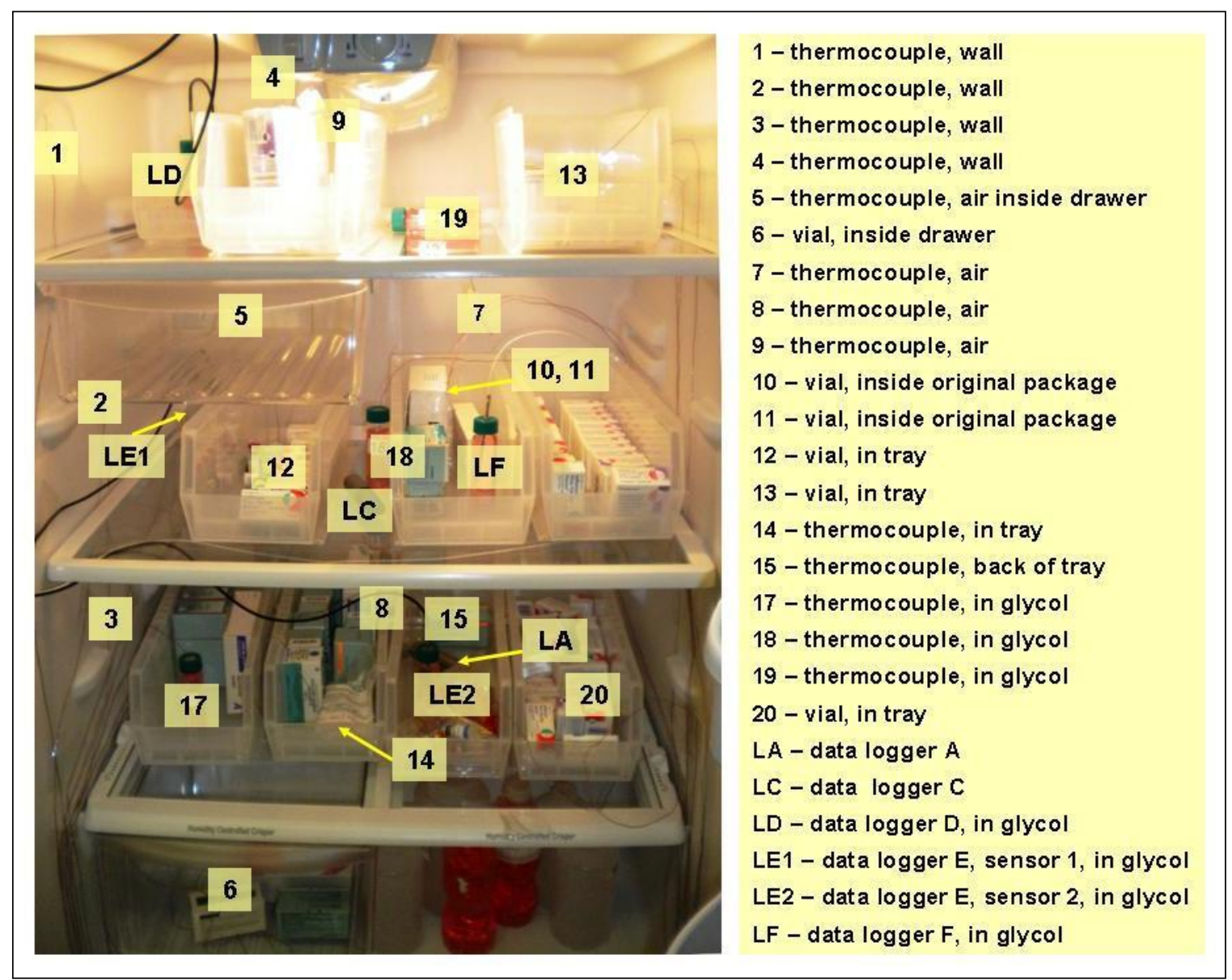

Fig. 1. Thermocouple and data logger installation pattern-dual-zone refrigerator. 


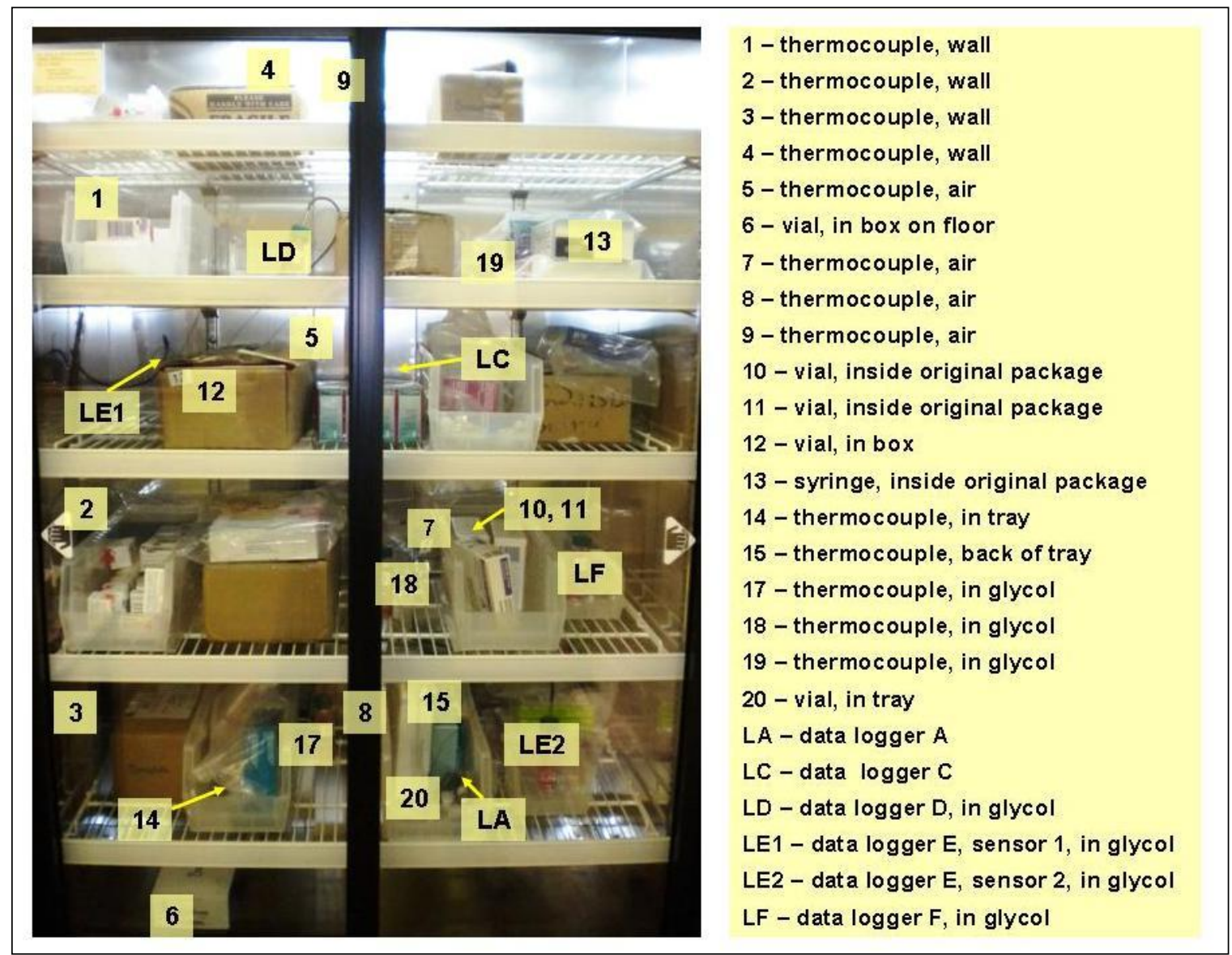

Fig. 2. Thermocouple and data logger installation pattern-pharmaceutical refrigerator.

Table 2. Equipment used for the present study.

\begin{tabular}{|c|c|}
\hline Equipment name: & Specifications: \\
\hline Dual-zone Refrigerator & $\begin{array}{l}\text { - Fully automatic cycle defrost refrigerator with top } \\
\text { freezer }(120 \mathrm{~V}) \\
\text { - Height: } 1.68 \mathrm{~m}[66.125 \mathrm{in} .] \\
\text { - Width: } 0.762 \mathrm{~m}[30 \mathrm{in} .] \\
\text { - } \text { Depth: } 0.759 \mathrm{~m}[29.875 \mathrm{in} .] \\
\text { - } \text { Total capacity: } 0.515 \mathrm{~m}^{3}[18.2 \mathrm{cu} \text {. ft.] } \\
\text { - } \text { cu. ft.] } \\
\text { - Forced convection of cooling air }\end{array}$ \\
\hline Pharmaceutical Refrigerator & $\begin{array}{l}\text { - Fully automatic cycle defrost refrigerator }(120 \mathrm{~V}) \\
\text { - Height: } 1.79 \mathrm{~m} \\
\text { - Width: } 0.9 \mathrm{~m} \\
\text { - } \text { Capth: } 0.6 \mathrm{~m} \\
\text { - } \text { Forced convection of cooling air } \\
\text { - Electronic control system and digital thermometer }\end{array}$ \\
\hline
\end{tabular}




\begin{tabular}{|c|c|}
\hline & - $\quad$ Temperature, air circulation and door alarms \\
\hline Plastic trays & - $\quad$ Stacking plastic storage trays/ bins \\
\hline 19 thermocouples & $\begin{array}{ll}\text { - } & \text { Type } \mathrm{T} \text { (copper-constantan) } \\
\text { - } & 0.5 \mathrm{~mm} \text { diameter, } 1.83 \mathrm{~m} \text { length } \\
\text { - } & \text { Teflon insulation } \\
\text { - } & \text { Calibrated at } 0{ }^{\circ} \mathrm{C}\end{array}$ \\
\hline Data logger A & $\begin{array}{l}\text { - } \text { Rugged high-temperature logger } \\
\text { - } \text { Metal housing with integral } 2.6 \mathrm{~cm} \text { rigid probe } \\
\text { - } \text { Temperature range: }-40{ }^{\circ} \mathrm{C} \text { to }+150{ }^{\circ} \mathrm{C} \\
\text { - } \text { Temperature resolution: } 0.05{ }^{\circ} \mathrm{C} \\
\text { - } \text { Calibrated accuracy: } \pm 0.5{ }^{\circ} \mathrm{C} \text { (from manufacturer) } \\
\text { - } \text { Memory: } 32,767 \text { readings } \\
\text { - Battery life: } 1 \text { year } \\
\text { - } \text { Body dimensions: } 12.2 \mathrm{~cm} \times 2.6 \mathrm{~cm} \text { dia. } \\
\end{array}$ \\
\hline Data logger $\mathrm{C}$ & $\begin{array}{l}\text { - } \text { Plastic-housing temperature and humidity Logger } \\
\text { - } \text { Temperature range: }-35^{\circ} \mathrm{C} \text { to } 80{ }^{\circ} \mathrm{C} \\
\text { - } \text { Temperature resolution: } 0.5{ }^{\circ} \mathrm{C} \\
\text { - } \text { Accuracy: } \pm 0.3{ }^{\circ} \mathrm{C} \text { (manufacturer rating) } \\
\text { - } \text { Battery life: } 1 \text { year } \\
\text { - Nominal body dimensions: } 10.2 \mathrm{~cm} \mathrm{x} 2.3 \mathrm{~cm} \text { max dia. }\end{array}$ \\
\hline Data logger D & 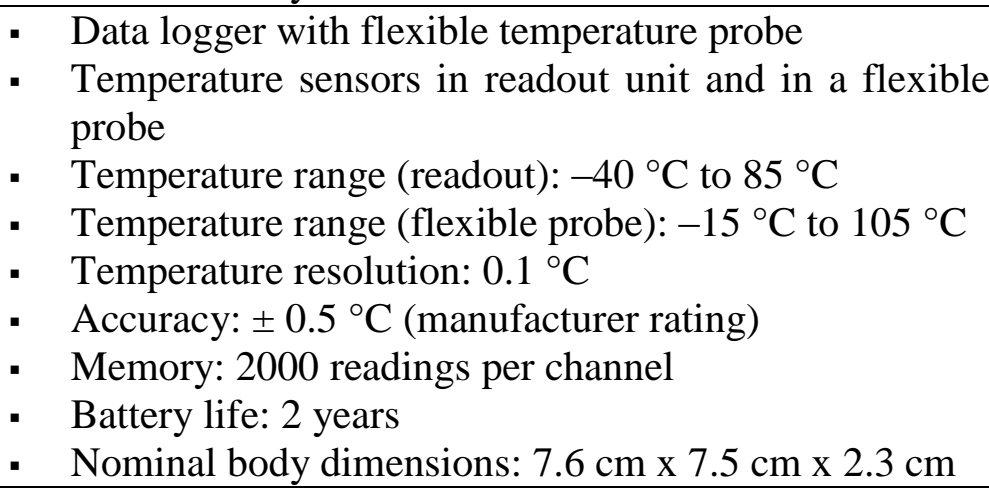 \\
\hline Data logger E1, E2 & 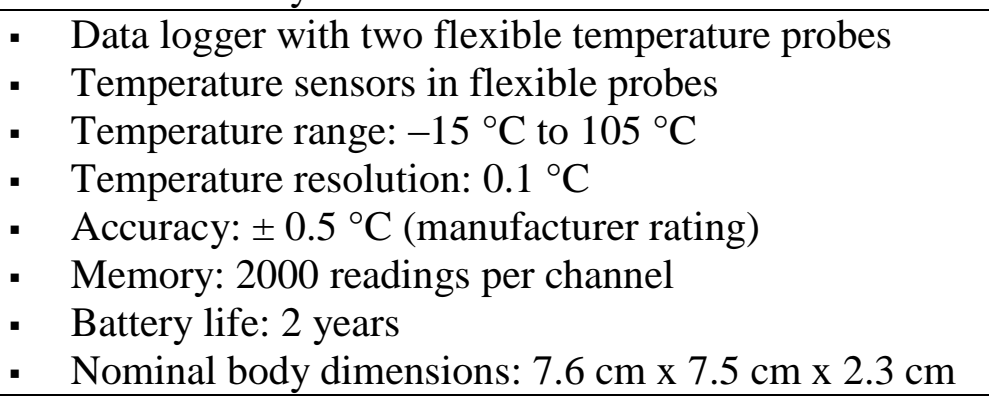 \\
\hline Data logger F & 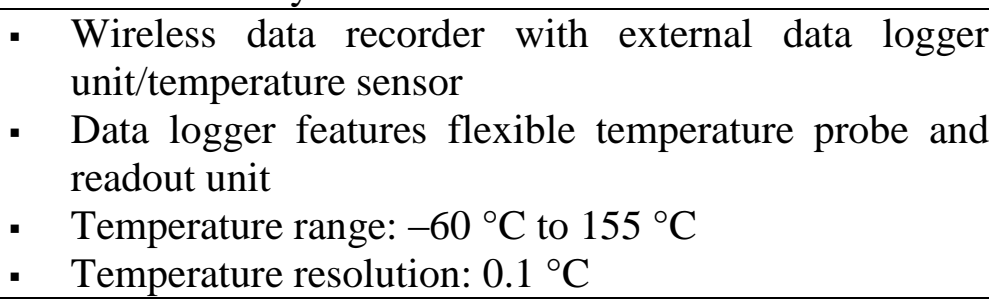 \\
\hline
\end{tabular}




\begin{tabular}{|c|c|}
\hline & $\begin{array}{l}\text { - Accuracy: } \pm 0.3{ }^{\circ} \mathrm{C} \text { from }-20{ }^{\circ} \mathrm{C} \text { to } 80^{\circ} \mathrm{C} \text { (mfc rating) } \\
\text { - } \text { Memory: } 16,000 \text { readings } \\
\text { - } \text { Battery life: } 6 \text { months } \\
\text { - } \text { Nominal body dimensions: } 6.2 \mathrm{~cm} \text { x } 4.7 \mathrm{~cm} \text { x } 1.9 \mathrm{~cm} \\
\text { (Lireless data transmission distance: Up to } 100 \text { Meters } \\
\text { (Light and No Obstructions) }\end{array}$ \\
\hline $\begin{array}{l}\text { Thermocouple measurement } \\
\text { system }\end{array}$ & - 6.5 digit multimeter with scanner \\
\hline
\end{tabular}

Table 3 contains the uncertainties corresponding to the measurement devices and systems used. To calculate the uncertainty,

Table 3. Measurement Device Uncertainties

\begin{tabular}{|l|c|}
\hline Device name: & $\boldsymbol{U}(\boldsymbol{k}=\mathbf{2}),{ }^{\circ} \mathbf{C}$ \\
\hline Thermocouple measurement system & 0.12 \\
\hline Data logger A & 0.58 \\
\hline Data logger B & 1.41 \\
\hline Data logger C & 0.67 \\
\hline Data logger D & 0.59 \\
\hline Data logger E1, E2 & 0.59 \\
\hline Data logger F & 0.37 \\
\hline
\end{tabular}

In general, each trial was started during the afternoon, and measurements recorded overnight and into the next morning. On average, we recorded 16 to $24 \mathrm{~h}$ of data for each trial, although some trials were continued over the course of several days. The only exception to this was the door opening trials, which only lasted 4 to $5 \mathrm{~h}$. The thermocouple measurement system records readings once every $10 \mathrm{~s}$. The individual data loggers can be set to various reading rates. In general, the loggers were set to record once every minute, except for logger $\mathrm{F}$, which recorded at $30 \mathrm{~s}$ intervals. During the door opening trials, we increased the reading rate to once every $10 \mathrm{~s}$ to $42 \mathrm{~s}$ (depending on the capabilities of the logger).

\section{INTERPRETATION OF RESULTS}

\subsection{Dual-Zone Refrigerator}

\subsubsection{Effect of loading density}

In trials performed using the dual-zone refrigerator, vaccine storage density had a slight impact on the refrigerator's ability to maintain the desired temperature range. The photographs below (Fig. 4) show the levels of packing density that were tested. 


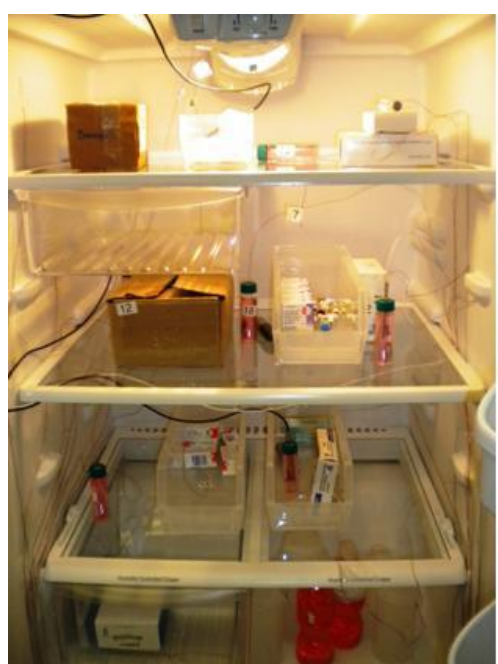

Low Density Pack

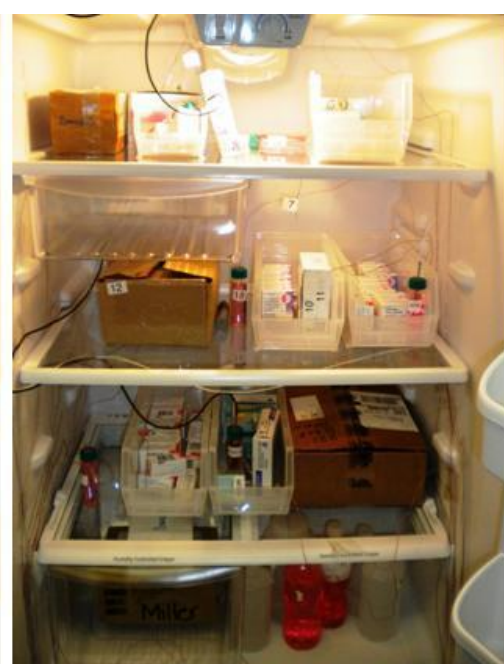

Medium Density Pack

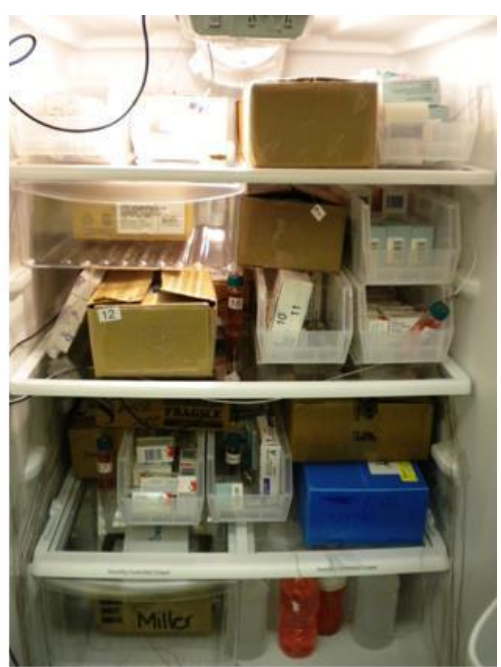

High Density Pack

Fig. 4. Typical packing patterns.

In general, the dual-zone refrigerator maintained a stable temperature profile throughout the different packing density configurations. During trials with higher density loads, the degree of location-specific temperature variation appeared to increase slightly. This is reflected in figure 5 below, as the vertical spread of the data is slightly larger in some of the trials with a high density loading scheme than in the lower density trials.

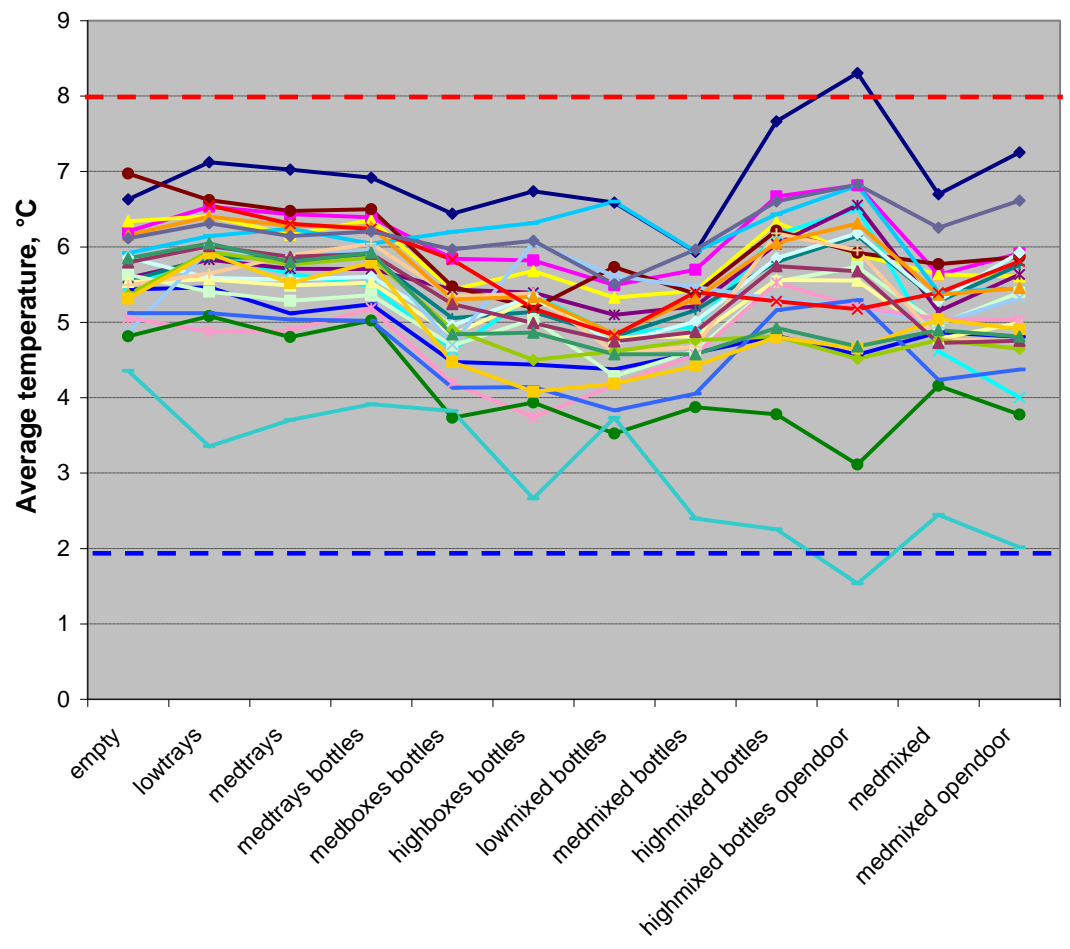

$\begin{aligned} &- 1 \text { (top wall) } \\ &- 2 \text { (mid wall) } \\ & 3 \text { (lower wall) } \\ & 4 \text { (top back wall) } \\ & \rightarrow 5 \text { (air - deli drawer) } \\ &- 6 \text { (vial - crisper drawer) } \\ &- 7 \text { (air) } \\ &-8 \text { (air) } \\ &-9 \text { (air - top) } \\ & 10 \text { (in box) } \\ &-11 \text { (in box) } \\ & 12 \text { (vial - mid) } \\ & 13 \text { (vial - top) } \\ & 14 \text { (inside tray) } \\ &-15 \text { (back of tray) } \\ & 17 \text { (glycol - low) } \\ &-18 \text { (glycol - mid) } \\ &-19 \text { (glycol - top) } \\ &-20 \text { (vial - low) } \\ & \text { LA (low, in tray) } \\ &- \text { LC (mid) } \\ &- \text { LD (glycol - top) } \\ &- \text { LE1 (glycol - mid) } \\ &- \text { LE2 (glycol - low) } \\ &\end{aligned}$


Fig. 5. Average temperature of freezerless refrigerator for all sensors, as a function of loading pattern. Labels on the abscissa correspond to entries in the measurement matrix of Table 1 .

Figure 5 shows the average reading for each thermometer across all of the closed-door dual-zone refrigerator trials, as well as for the periodic door-opening trials. The majority of the thermometers recorded average temperatures within the prescribed $2{ }^{\circ} \mathrm{C}$ to $8{ }^{\circ} \mathrm{C}$ window across the range of testing, regardless of whether the refrigerator load was low, medium, or high density. The average temperatures of the sensors attached to vaccine vials did not vary by more than $1.7^{\circ} \mathrm{C}$ between trials, remaining centered around $5^{\circ} \mathrm{C}$ to $6{ }^{\circ} \mathrm{C}$ throughout the study.

However, during the high density, door-opening trial, two thermometers recorded average temperatures outside the prescribed range. Thermometer 1 , attached to the top wall of the refrigerator, recorded an average temperature of $8.3{ }^{\circ} \mathrm{C}$, while thermometer 19, a thermocouple inside a glycol-filled bottle on the top refrigerator shelf, recorded an average temperature of $1.5^{\circ} \mathrm{C}$ for the trial. Both of these thermometers displayed outlier behavior across the range of testing, and therefore are not good indicators actual vaccine vial of properly stored vial temperatures. The dual-zone refrigerator model tested featured a cold air vent centered at the top wall of the refrigerator space. The glycol-filled bottle containing thermocouple 19 was positioned directly on the glass shelf underneath this cold air vent. As a result, this thermometer consistently recorded temperatures $2{ }^{\circ} \mathrm{C}$ to 5 ${ }^{\circ} \mathrm{C}$ colder than those in the rest of the refrigerator space. By contrast, thermometer 1 was taped to the top refrigerator wall, away from the cooling vent, causing it to record slightly warmer temperatures than thermometers in the main body of the refrigerator. In both cases, thermometers 1 and 19 showed the largest deviations during the high density loading schemes. Because the refrigerator's air circulation patterns are inhibited by higher density loads, pockets of warmer and cooler air are formed. This effect is magnified for the less ideal locations, such as the top refrigerator walls (where warm air rises) and directly under the cooling vent. However, for the temperature sensors placed throughout the rest of the refrigerator space, including those attached to vials, the increase in location-specific temperature variation at high density loads was not pronounced enough to result in thermal excursions.

Considering a refrigerator used for everyday vaccine storage, the arrival of a large vaccine shipment or the onset of influenza season is likely to result in a sudden increase or decrease in the amount of material stored in the refrigerator. Because these types of load density variations did not greatly affect overall temperature stability, the dual-zone refrigerator model appears to be suitable for normal vaccine storage. However, care should be taken to avoid storing vaccines directly under the cooling vent. An unpackaged vaccine placed in this location may be susceptible to thermal excursions below $2{ }^{\circ} \mathrm{C}$, particularly during a time of high density refrigerator loading. 


\subsubsection{Effect of packing style}

Changing the vaccine packing style did not have a noticeable impact on the overall refrigerator and vial temperature stability, whether plastic trays, cardboard boxes, or a combination of both were used to store the vaccines. (See Fig. 6.) While official guidelines for vaccine storage mandate that vials be stored in permanent trays or containers and organized by type, we observed other storage conditions during our visits to the VFC clinics. For example, a busy clinic with a sudden influx of vaccines might temporarily store vaccines inside their shipping boxes. The measurement results indicate that the dual-zone refrigerator model would be able to withstand this practice, continuing to preserve the prescribed vaccine storage temperature range regardless of packaging style.

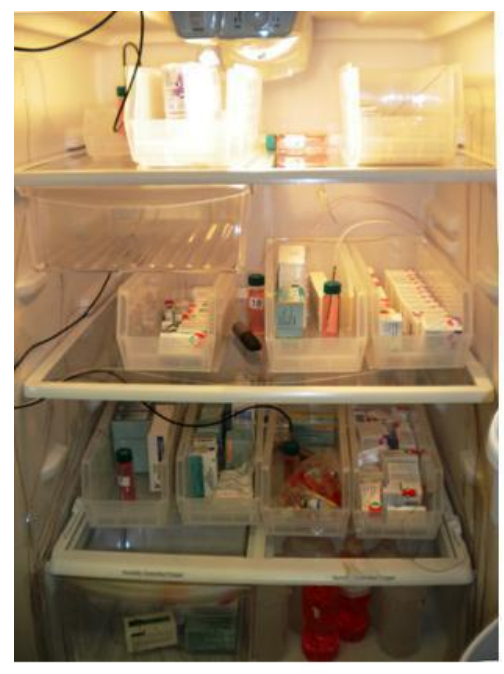

Plastic Trays

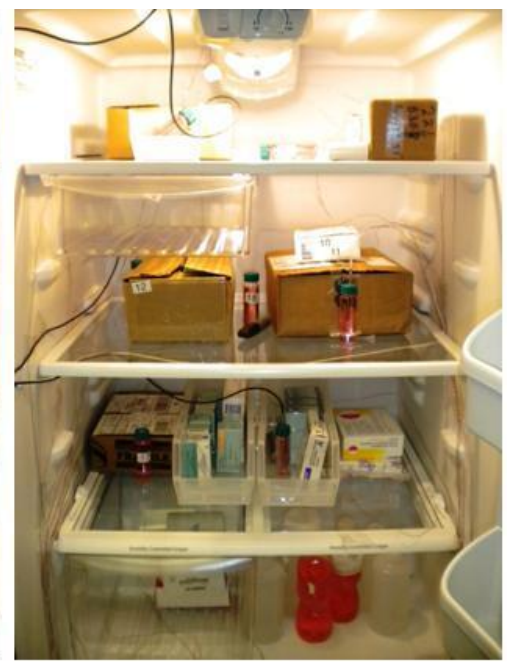

Cardboard Boxes

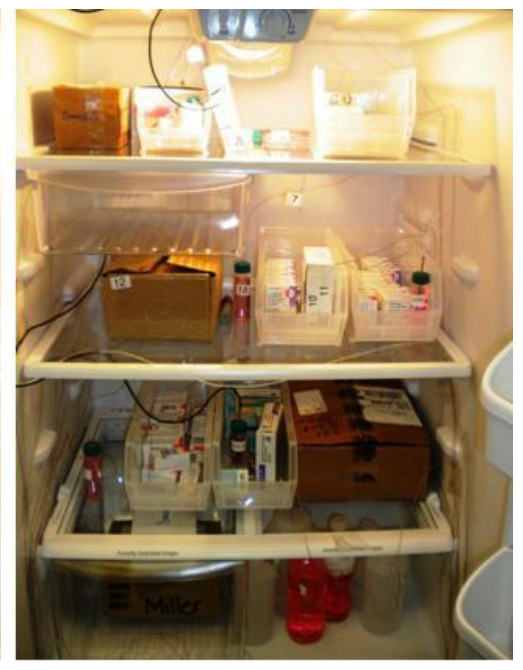

Mixed Trays and Boxes

Fig. 6. Various vaccine storage methods.

\subsubsection{Effect of door opening and closing —normal use simulation}

During the trials in which the refrigerator door was periodically opened and closed to simulate a normal use pattern, the dual-zone refrigerator successfully kept monitored vaccine vials within the $2{ }^{\circ} \mathrm{C}$ to $8{ }^{\circ} \mathrm{C}$ range. The overall impact of the door opening on vaccine vial temperature was quite small. For the sensors attached to vials, the mean temperature difference between the closed door and door-opening trials was $0.6{ }^{\circ} \mathrm{C}$ or less. However, well over one-third of all the remaining thermometers recorded large thermal excursions both above and below the prescribed temperature bounds during the door-opening trials. Figure 7 below shows the temperature responses of each of the thermocouples during a door-opening trial. 


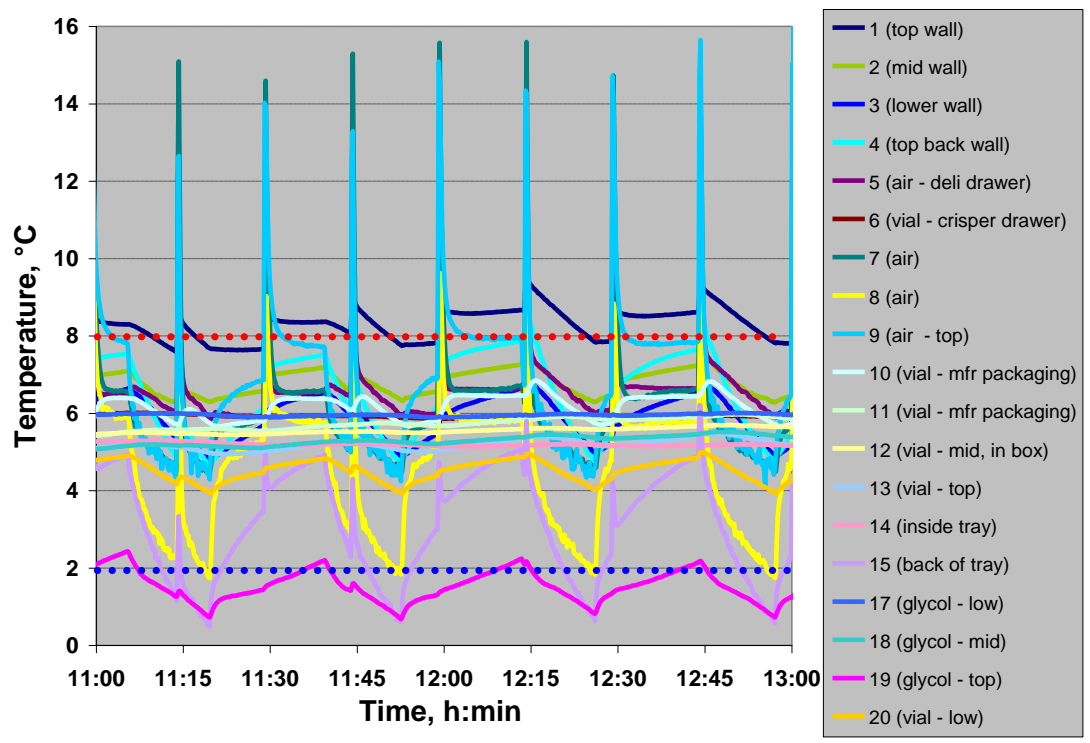

Fig. 7. Door opening trial for the dual-zone refrigerator using a high density mixed load with water bottles.

During the dual-zone refrigerator tests, we found that the temperature behavior of different sensors during door opening and closing trials varied drastically depending on sensor location and installation method. The results in the figure above show some of the sensors recording a large temperature spike each time the door is opened, followed by a dip that in some cases falls below $2{ }^{\circ} \mathrm{C}$. The thermometers that displayed this type of temperature behavior were attached to the upper refrigerator walls or hanging in the open airspace. Another group of sensors recorded temperatures clustered around $5{ }^{\circ} \mathrm{C}$ to $6{ }^{\circ} \mathrm{C}$ for the duration of each trial, showing comparatively little response to the door opening and closing. This group consisted of sensors attached to vaccine vials as well as those placed inside glycol-filled bottles stored in the center of the refrigerator. Figure 8a, below left, shows the data collected by these sensors only.
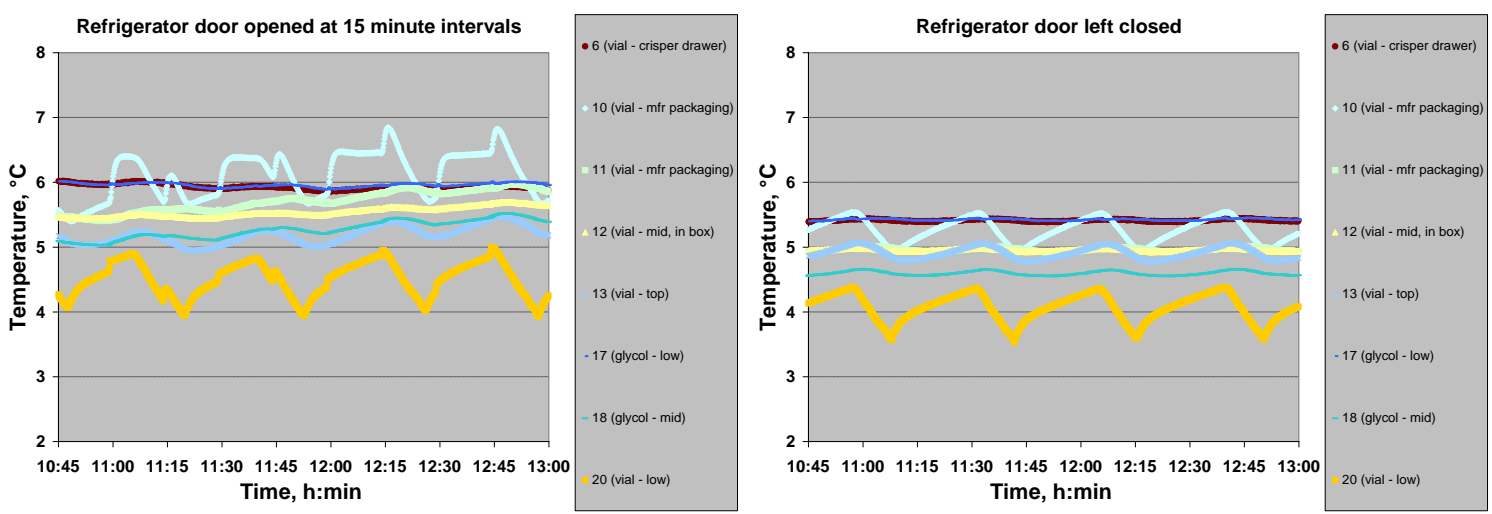

Fig. 8a. (Left) Door opening trial for the dual-zone refrigerator. Only thermocouples monitoring vial temperatures or inside glycol-filled bottles are shown.

Fig. 8b. (Right) Temperature response of same selected thermocouples, recorded $24 \mathrm{~h}$ later and with refrigerator door kept closed. 
Note: Due its placement under the cooling vent, thermocouple \#19 recorded temperatures up to $6{ }^{\circ} \mathrm{C}$ colder than monitored vials and other sensors in glycol-filled bottles. Because of this, TC \#19 is excluded from these graphs.

Figure 8a shows the response of the selected thermometers during one of the periodic door-opening trials, while figure $8 \mathrm{~b}$ shows the closed-door, equilibrium temperature behavior of the same thermometers, for comparison. It is apparent from the figures that while the normal use pattern of opening and closing the refrigerator door does have an effect on the temperatures experienced by stored vaccines, the effect is not likely to be large enough to threaten the potency of the vaccines via large thermal excursions. These findings support the use of the dual-zone refrigerator model as an effective storage system for refrigerated vaccines under normal operating conditions.

While the vaccine vial temperatures remained relatively stable throughout the dooropening trials, a large percentage of the temperature sensors arranged throughout the refrigerator recorded an entirely different picture. The large temperature fluctuations recorded by some of the thermocouples hanging in the air or attached to the refrigerator walls underscore the importance of choosing the proper temperature monitoring location and method.

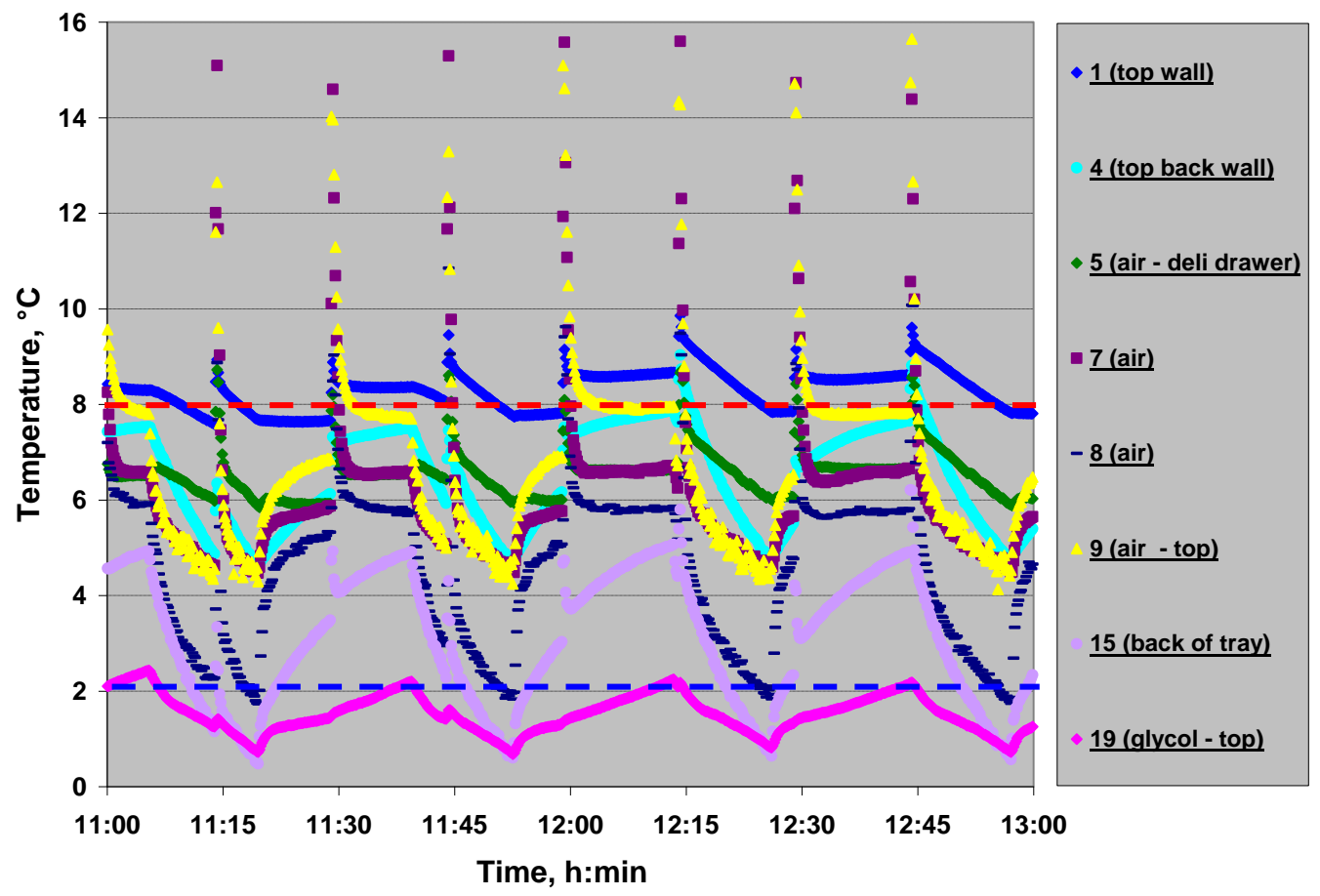

Fig. 9. Door opening trial for the dual-zone refrigerator, showing only thermocouples which recorded out-of-range data.

Figure 9 above shows data taken concurrently with that in figure $8 \mathrm{a}$, but only the sensors that recorded temperatures outside the $2{ }^{\circ} \mathrm{C}$ to $8{ }^{\circ} \mathrm{C}$ bounds are shown. Each of these sensors, with the exception of sensor 19 , recorded a large temperature spike each time the refrigerator door was opened. The sensors hanging in the open air $(7,8,9)$ saw the most 
dramatic spikes, with temperature increases of $5{ }^{\circ} \mathrm{C}$ to $10{ }^{\circ} \mathrm{C}$ higher than their previous readings. A provider using a similar setup to monitor their vaccine storage system, such as a thermometer set up to record refrigerator air temperature, would most likely obtain a similar result. Every time a worker briefly opened the refrigerator door, the thermometer would likely record a large temperature spike exceeding the $8{ }^{\circ} \mathrm{C}$ limit. Although this temperature data would suggest that the vaccines were subjected to improper storage conditions, we saw in the results of our study that the thermal state of the vaccine vials remained relatively stable despite fluctuations in the refrigerator air temperature brought on by repeated door opening.

It is clear that choosing the right thermometer and correctly positioning it inside the refrigerator is critical to obtaining meaningful temperature monitoring data. The obvious purpose of a vaccine refrigeration system is to maintain vaccine vials and packages at a temperature that is within $2{ }^{\circ} \mathrm{C}$ to $8{ }^{\circ} \mathrm{C}$, while the goal of a vaccine temperature monitoring system is to provide quantitative evidence that the vaccines have in fact been kept within this range. During the course of normal refrigerator use, such as when the door is briefly opened and closed to add or remove stock, the interior refrigerator air temperature will experience fluctuations that are much larger or do not correspond to the temperature effects experienced by vaccines. A vaccine usually consists of liquid inside a glass bottle or plastic syringe, which is often contained in some type of cardboard packaging. As a result, a packaged vaccine has a much larger thermal mass than the same volume of air. This means that it takes the vaccines much longer to thermally "see" the effects of conditions like opening the refrigerator door.

A thermometer with a thermal mass similar to a stored vaccine can be used as an effective tool for determining vaccine temperature. If the thermometer probe itself does not meet this criterion, placing the probe inside a bottle filled with a liquid, such as glycol, is a simple way to mimic the temperature behavior of a vaccine inside a bottle. During these door opening trials, we found that the data from both the thermocouples and data loggers with probe placed inside glycol-filled bottles closely followed the results of the thermocouples attached to vaccine vials. The one exception to this was thermocouple 19, shown in figure 9 above.

Thermocouple 19 was contained inside a glycol-filled bottle and placed on the refrigerator shelf, near to stored vaccines. The other thermocouples in glycol, 17 and 18, recorded temperature data that mirrored the results of the vial-attached thermocouples throughout the range of testing. However, thermocouple 19 consistently recorded temperatures $2{ }^{\circ} \mathrm{C}$ to $5{ }^{\circ} \mathrm{C}$ colder than any of the other sensors across all of the trials. The difference lies in the thermocouple's location inside the refrigerator. The glycol-filled bottle containing thermocouple 19 was placed on its side, directly on the upper glass refrigerator shelf. Located directly above this spot was the refrigerator cooling vent. This means that every time the refrigerator compressor cycled on, cold air was blown directly onto both the glass shelf and the bottle containing thermocouple 19. This clearly explains why the temperatures recorded from this spot were so much colder than from other areas of the refrigerator. During the door-opening trials, the repeated influx of roomtemperature air likely caused the refrigerator to pump out additional cold air to 
compensate. This would in turn drive the temperatures of the shelf and the sensors near the vent down even lower. Our data supports this explanation, as the lowest temperatures recorded at any point in the study were measured by thermocouple 19 during the door opening trials.

During trials using the freezerless and dorm-style refrigerator models, we found that storing water bottles in the door of the unit improved overall temperature stability by reducing the impact of normal use conditions like opening the refrigerator door [1]. However, in trials of the dual-zone model, the difference in performance between the refrigerator with water bottles vs. without water bottles was less pronounced. Table 4 below shows the different door opening temperature changes for the two trials, with and without water bottles. In this case, the temperature change is defined as the difference between average temperature recorded during the closed door trial, and the average temperature from the door-opening trial with the same loading configuration. The closed door trial temperature averages were taken from a $2 \mathrm{~h}$ representative sample of data. The temperature averages from door-opening trial were taken over the entire duration of the trial, which was $2 \mathrm{~h}$.

Table 4. Temperature changes during door-opening tests (all values in degrees Celsius).

\begin{tabular}{|c|c|c|}
\hline $\begin{array}{c}\text { Sensor } \\
\text { Name/Location }\end{array}$ & $\begin{array}{l}\text { High Density Mixed } \\
\text { With Bottles }\end{array}$ & $\begin{array}{c}\text { Medium Density Mixed } \\
\text { No Bottles }\end{array}$ \\
\hline 1 (top wall) & 0.6423 & 0.5537 \\
\hline 2 (mid wall) & 0.1467 & 0.2842 \\
\hline 3 (lower wall) & -0.4250 & -0.0251 \\
\hline 4 (top back wall) & 0.3464 & -0.6174 \\
\hline 5 (air - deli drawer) & 0.5182 & 0.4871 \\
\hline 6 (vial - crisper drawer) & -0.2926 & 0.0958 \\
\hline 7 (air) & 0.3457 & 0.4844 \\
\hline 8 (air) & -0.2525 & -0.0609 \\
\hline 9 (air - top) & 0.3814 & 0.0494 \\
\hline 10 (in box) & 0.3294 & 0.6409 \\
\hline 11 (in box) & 0.1940 & 0.4255 \\
\hline 12 (vial - mid) & -0.0106 & 0.2154 \\
\hline 13 (vial - top) & -0.1090 & 0.3425 \\
\hline 14 (inside tray) & -0.3291 & -0.0145 \\
\hline 15 (back of tray) & -0.6643 & -0.3806 \\
\hline 17 (glycol - low) & -0.2242 & -0.0264 \\
\hline 18 (glycol - mid) & 0.1346 & 0.1369 \\
\hline 19 (glycol - top) & -0.7202 & -0.4313 \\
\hline 20 (vial - low) & -0.2985 & -0.1190 \\
\hline LA (low, in tray) & -0.1531 & -0.1330 \\
\hline LC (mid) & 0.2551 & 0.0868 \\
\hline LD (glycol - top) & -0.1058 & 0.4173 \\
\hline LE1 (glycol - mid) & -0.0676 & 0.0293 \\
\hline LE2 (glycol - low) & -0.2455 & -0.0867 \\
\hline LF (glycol - mid) & 0.2230 & 0.3599 \\
\hline
\end{tabular}

The negative temperature change of many of the sensors indicates that the average recorded temperature was lower during the door opening trial than in the closed door trial. Although room temperature air entering the refrigerator each time the door is opened causes the internal temperature of the refrigerator to rise, the cooling unit compensates by pumping in additional cold air. From our results, it appears that the dual- 
zone cooling unit may over-compensate slightly, driving the temperature several tenths of a degree below the original set point.

Twenty-one out of the twenty six sensors recorded a smaller temperature change when water bottles were used in the door. However, this effect was very small, amounting to a difference of half a degree at most.

The effect of the water bottles was much more pronounced during tests in which the refrigerator door was left cracked open for $1 \mathrm{~h}$, to simulate an employee forgetting to completely close the door. Table 5 below shows the amount of time elapsed once the door was opened until each of the vials exceeded the allowed maximum temperature of $8{ }^{\circ} \mathrm{C}$.

Table 5. Time after opening door until vial temperature exceeded $8{ }^{\circ} \mathrm{C}$.

\begin{tabular}{|l|c|c|}
\hline \multicolumn{1}{|c|}{ Name and Location } & $\begin{array}{c}\text { High Density Mixed } \\
\text { With Bottles } \\
\text { (minutes) }\end{array}$ & $\begin{array}{c}\text { Medium Density Mixed } \\
\text { No Bottles } \\
\text { (minutes) }\end{array}$ \\
\hline vial 10 (inside original packaging) & 5 & 4 \\
vial 11 (inside original packaging) & 24 & 18 \\
vial 13 (in tray, upper shelf) & 27 & 3 \\
vial 12 (inside cardboard box) & $62^{*}$ & 58 \\
vial 20 (in tray, lower shelf) & did not exceed & 32 \\
vial 6 (inside box, in vegetable crisper) & did not exceed & 53 \\
\hline
\end{tabular}

$*$ door closed after 60 minutes

All of the vials resisted thermal excursions above $8{ }^{\circ} \mathrm{C}$ for a longer period of time when the water bottles were in place. For two of the monitored vaccine vials, the effect of the water bottle ballast was significant enough to prevent any exposure to out-of-range temperatures.

\subsubsection{Effect of Increasing Room Temperature}

Although vaccine storage refrigerators should be kept in a fairly well temperature controlled room, considerable ambient temperature fluctuations may result from planned HVAC outages at night or weekends, or from unplanned circumstances such as an AC outage or poor thermostat control. To determine whether these fluctuations impact the dual-zone refrigerator's ability to maintain its set point temperature, we performed three trials in which the room temperature was varied between $18{ }^{\circ} \mathrm{C}$ and $24^{\circ} \mathrm{C}$.

Data logger LD has a thermometer included in the main readout unit as well as an additional probe attached via cable. This probe was inserted into a glycol-filled bottle kept inside the refrigerator throughout all of the trials, while the readout unit was kept outside of the refrigerator, in a tray taped to the refrigerator door. In this way, we were able to simultaneously measure both the internal refrigerator temperature and the external ambient temperature using the same data logger. By comparing the relationship between internal refrigerator and ambient temperature, we can determine the effect an increase in room temperature can have on vaccine storage conditions. Data loggers LE1 and LE2 
have the same specifications as logger LD, and both of these loggers also had temperature probes kept in glycol-filled bottles inside the refrigerator. Because of this, we have also included the readings from LE1 and LE2 in the comparison analysis to better establish the relationship between ambient temperature and its effects on interior refrigerator temperature control.

Table 6 shows the temperature change recorded by each of the three refrigerator data logger probes scaled to the change in ambient temperature recorded by the external probe. So, for example, during the High Density Mixed with Bottles I trial, logger LE1 recorded a temperature decrease of $0.02{ }^{\circ} \mathrm{C}$ for every one degree increase in room temperature.

Table 6. Refrigerator probe temperature change divided by room temperature change $\left({ }^{\circ} \mathrm{C}\right.$ $\left./{ }^{\circ} \mathrm{C}\right)$

\begin{tabular}{|c|c|c|c|}
\hline Logger Name & $\begin{array}{c}\text { High Density Mixed } \\
\text { With Bottles I }\end{array}$ & $\begin{array}{c}\text { High Density Mixed } \\
\text { With Bottles II }\end{array}$ & $\begin{array}{c}\text { Medium Density Mixed } \\
\text { No Bottles }\end{array}$ \\
\hline LD & 0.00 & -0.03 & 0.04 \\
LE1 & -0.02 & -0.03 & -0.04 \\
LE2 & -0.05 & -0.10 & -0.05 \\
\hline
\end{tabular}

During trials using the freezerless refrigerator, we saw that room temperature and internal refrigerator temperature were directly related. For every degree rise in room temperature, the temperature inside the refrigerator also increased by some fractional amount. However, in trials using the dual-zone refrigerator, the relationship between room temperature and internal refrigerator temperature proved to be much more complex. Most of the data in table 6 above suggests that there is an inverse relationship between room temperature and internal refrigerator temperature. This may be a product of refrigerator's internal temperature control, which increases cooling power in response to the warmer environmental conditions. However, not all of the data fits within this paradigm. During the Medium Density Mixed load trial, logger LD recorded a temperature increase of 0.04 ${ }^{\circ} \mathrm{C}$ per degree rise in room temperature. These inconsistencies are evidence that the complex relationship between exterior and interior refrigerator cannot be sufficiently captured by our limited investigation.

A number of extenuating factors, such as refrigerator load density, timing of a cooling or defrost cycle, initial refrigerator temperature, and the time rate of change of room temperature, impact the resulting temperature response of the refrigerator. Because we did not investigate the full scope of these factors, we will not attempt to delineate the exact relationship between room temperature change and its effect on refrigerator temperature control. However, we can make some generalizations about the overall impact of room temperature variation on the performance of a dual-zone refrigerator in terms of its role in a vaccine storage system.

First, and most importantly, the impact of changing room temperature on the dual-zone refrigerator is small. At the high end of the spectrum, we saw data logger LE2 record a temperature decrease of $0.1{ }^{\circ} \mathrm{C}$ per one degree increase in room temperature during one of the three trials. The remaining data sets showed an interior refrigerator temperature 
change of $0.05{ }^{\circ} \mathrm{C}$ or less per degree change in ambient temperature across all of the trials. By extrapolation, this means that environmental conditions must vary by $>20{ }^{\circ} \mathrm{C}$ for the items stored inside the refrigerator to experience just one degree of temperature change. By this analysis, if the initial refrigerator set point is set correctly, a $20{ }^{\circ} \mathrm{C}$ room temperature change is not likely to impact the efficacy of stored vaccines.

The dual-zone refrigerator performance during these varying room temperature tests demonstrates that the unit is able to withstand moderate fluctuations in ambient thermal conditions without sacrificing its ability to maintain proper vaccine storage temperatures. Only severe environmental temperature fluctuations are likely to have a significant impact on this refrigerator model's performance. For example, if a clinic in a northern climate experienced a heating outage during a snowstorm, the resulting room temperature drop might be enough to produce unacceptable thermal excursions inside the unit.

It is important to note that because these assertions are based on the observed effect of a $6{ }^{\circ} \mathrm{C}$ room temperature change, there may be additional thermal effects that occur under larger temperature swings. Any given refrigerator model may be subject to certain threshold operating conditions. If these conditions are exceeded, the refrigerator performance could be altered in an unexpected way. One way to effectively mitigate this risk is through the use of a continuous temperature monitoring system. This type of system puts the provider in control of their vaccine stock, alerting them of the status of their refrigerator at all times, under any environmental conditions. The data from the temperature monitoring device could then be used to identify possible weaknesses of the refrigeration system, such as inferior performance during moderate or extreme ambient temperature changes. Once these individualized weaknesses are identified, clinic workers will have the necessary tools to develop improved contingency plans, thereby enhancing the confidence of vaccine suppliers and providers, as well as the recipients, in the efficacy of delivered vaccines.

\subsubsection{Effect of a Power Outage}

To observe what occurs following a power outage, we performed two trials in which the closed refrigerator was unplugged and monitored overnight. During these trials, the room temperature was stable to within $1.5^{\circ} \mathrm{C}$. In both trials, all of the thermometers recorded a fairly steep increase in temperature that slowed down slightly over time. All of the thermometers, including those attached to vaccine vials, exceeded the allowed maximum temperature in less than $3 \mathrm{~h}$ for the medium density (no bottles) trial, and in $5 \mathrm{~h}$ in the high density with bottles trial. Figure 10 below shows the temperature readings of all of the thermometers during one of the power off trials. The sudden drop in temperature following the peak corresponds to the point at which the refrigerator was plugged back in. 

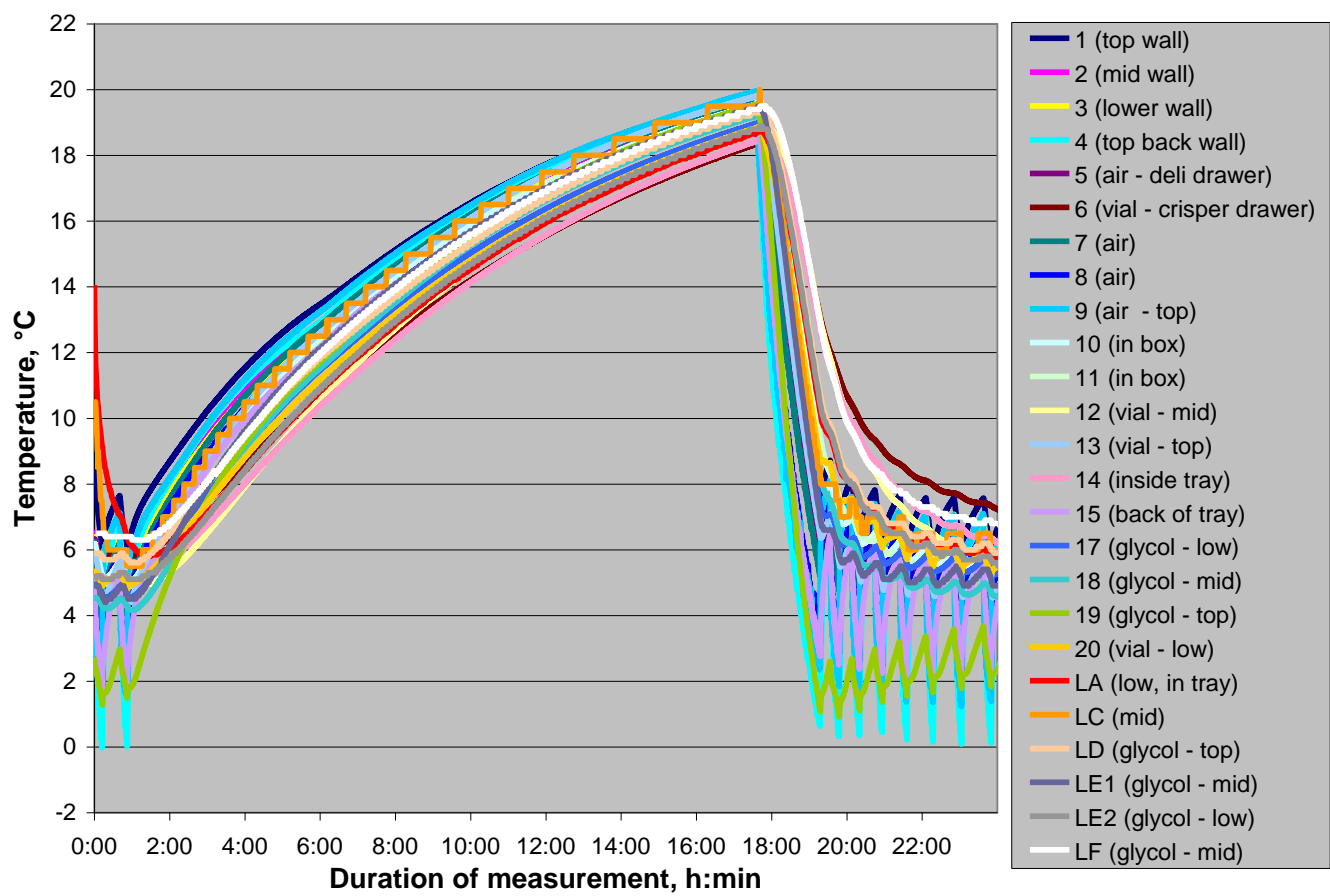

Fig. 10. Thermometer response during a power outage event. Medium density mixed load, no water bottles trial.

The next graph (Fig. 11) shows the data collected starting from the time that the refrigerator was unplugged. The dashed red line indicates the $8{ }^{\circ} \mathrm{C}$ upper limit, and the blue dashed line shows the $2{ }^{\circ} \mathrm{C}$ minimum.

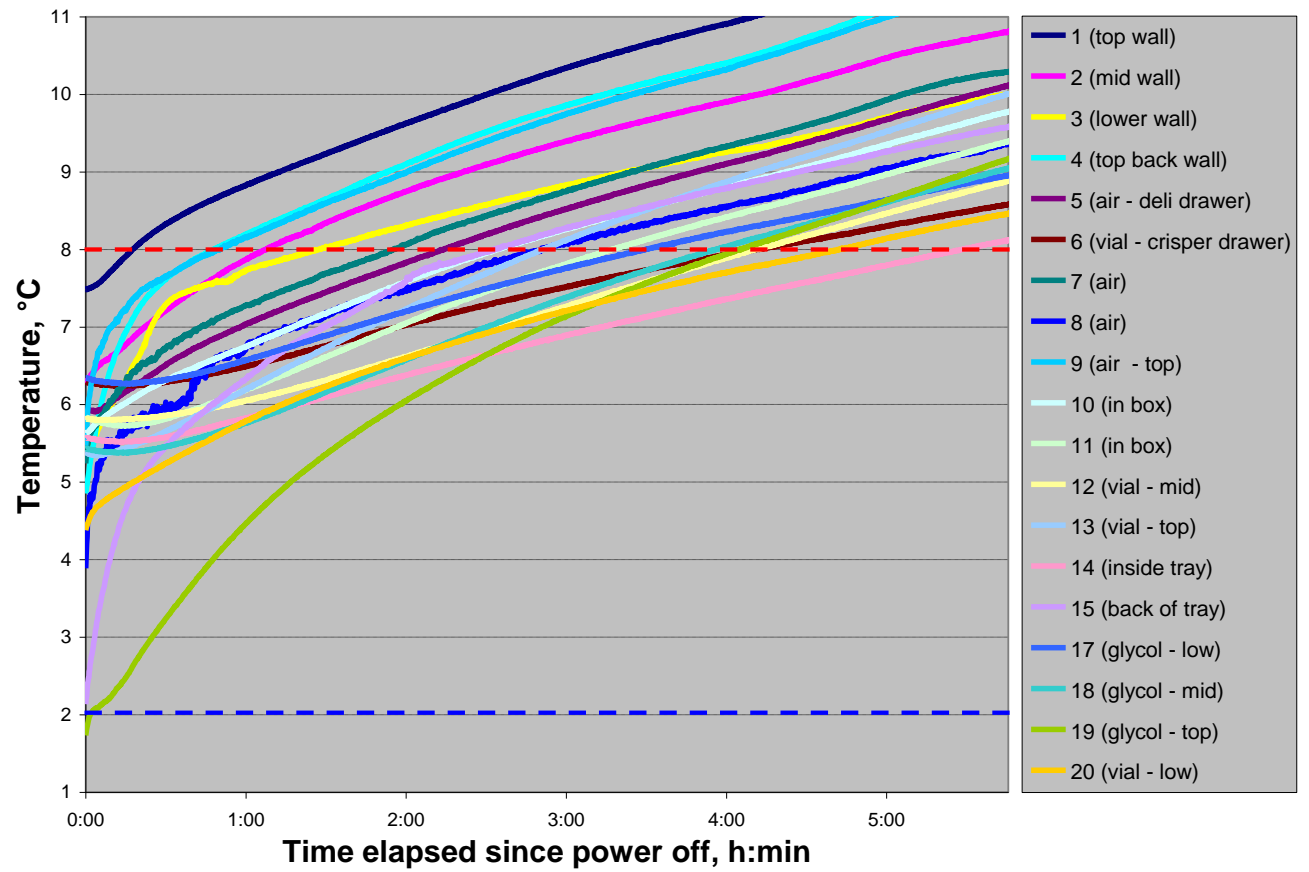

Fig. 11. Expanded view of thermometer response during a power-outage event. High density mixed load with bottles trial. 
To determine the required response time to preserve vaccine viability during a power outage situation, we examined the amount of time elapsed from the point that the refrigerator was unplugged until the thermometers attached to vials exceeded $8{ }^{\circ} \mathrm{C}$. This information is reported in Table 7 below.

Table 7. Time after power-off until vial temperature exceeded $8{ }^{\circ} \mathrm{C}$.

\begin{tabular}{|l|c|c|}
\hline \multicolumn{1}{|c|}{ Name and Location } & $\begin{array}{c}\text { High Density Mixed } \\
\text { With Bottles } \\
\text { (hrs:mins) }\end{array}$ & $\begin{array}{c}\text { Medium Density Mixed } \\
\text { No Bottles } \\
\text { (hrs:mins) }\end{array}$ \\
\hline vial 13 (in tray, upper shelf) & $2: 51$ & $1: 16$ \\
vial 10 (inside original packaging, middle shelf) & $2: 34$ & $1: 18$ \\
vial 11 (inside original packaging, middle shelf) & $3: 16$ & $1: 44$ \\
vial 20 (in tray, lower shelf) & $4: 42$ & $2: 05$ \\
vial 6 (inside box, in vegetable crisper) & $4: 10$ & $2: 08$ \\
vial 12 (inside cardboard box, middle shelf) & $4: 11$ & $2: 43$ \\
\hline
\end{tabular}

We can make several pertinent observations about this data. Of the two vials that were not contained in boxes or packaging material, the vial stored on a upper refrigerator shelf exceeded $8{ }^{\circ} \mathrm{C}$ faster than the vial on a lower shelf in both trials. This is expected response. With the power off, the fans do not circulate air throughout the cavity, so the warmer air rises to the top of the refrigerator. Hence, vials stored in the top portion of the refrigerator are exposed to warmer air than those stored in the bottom. This effect will be magnified for vials that are left exposed to air without the additional thermal mass provided by packaging material. Overall, the vials contained inside a thicker cardboard box resisted thermal excursions for a significantly longer time period than either the completely unpackaged vials or the vials kept only in their original thin cardboard packaging. This is because the thermal energy first must be conducted through layers of cardboard before it is able to heat up a vial inside a box.

Even so, these findings do not signify that all providers should package their vaccine vials in multiple layers of cardboard boxes to protect against thermal excursions during power outages. The flip-side of the coin is that as additional layers of packaging materials are used to store vaccine vials, the amount of time required to remove vaccines from the refrigerator is likely to increase. This could translate to workers spending additional time in front of the refrigerator with the door open, and as a result, a greater chance of thermal excursions caused by exposure to room temperature air. Because of this, it is critical to evaluate the best possible storage solutions depending on a provider's particular needs. For example, large, modern hospitals are likely to have backup generator systems to keep medical refrigerators running through power outages. Providers operating in this type of setting are likely to see many patients per day and have a high vaccine turnover rate, in which nurses must open and close vaccine storage refrigerators many times per day. In this case, minimizing the amount of time that the refrigerator door is open may be a more critical concern than protecting against power outages. If, by contrast, a provider office does not have a reliable backup generator and sees a comparatively small number of vaccine-related appointments, it might be worthwhile for them to package their stock more carefully to provide an extra safety margin in the event of a power outage. 
The results shown in table 7 clearly support the value of using water bottles as a temperature ballast. During the trial with water bottles in place, each of the vials resisted temperature increase above $8{ }^{\circ} \mathrm{C}$ for an extra $32 \mathrm{~min}$ to $2 \mathrm{~h} 37 \mathrm{~min}$ as compared to the trial without water bottles.

In order to observe the time required for the refrigerator to return to equilibrium following a power outage, we continued recording data after the refrigerator was powered. Figure 12 shows the data collected while the refrigerator cooled down back to its pre-outage temperature state.

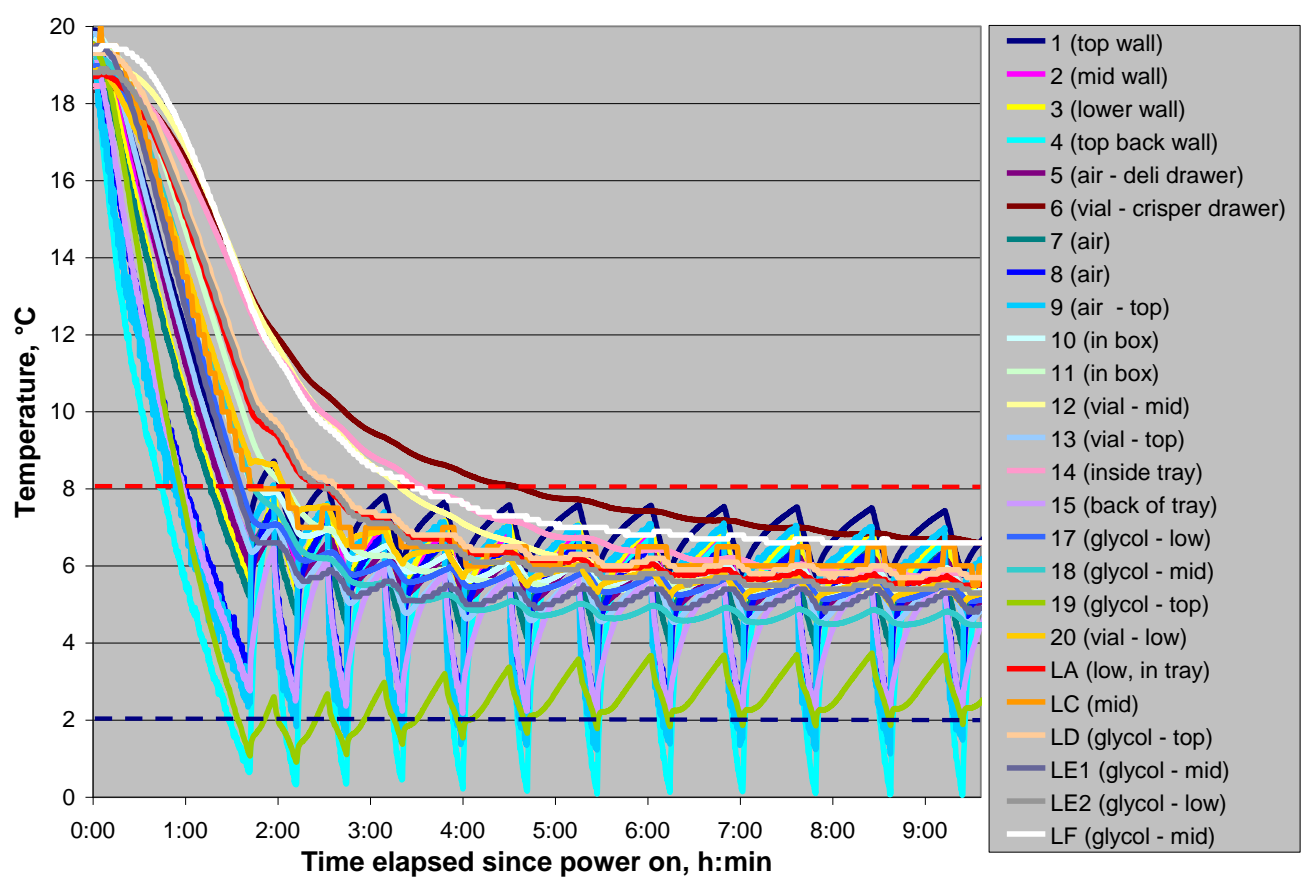

Fig. 12. Recovery after restoration of power for a dual-zone refrigerator.

The length of the power outage and the resulting maximum temperature attained will affect the length of time required to get the refrigerator back down to its original baseline. During the high density mixed load (including water bottles) trial, the refrigerator was left unplugged for 3 days, $17 \mathrm{~h} 28 \mathrm{~min}$. During the medium density mixed load trial, the total power off time was $16 \mathrm{~h} 16 \mathrm{~min}$. Because of this, the refrigerator reached a different maximum temperature in each trial, so the effect of loading density and water bottles on power outage recovery time cannot be evaluated based on these trials. The main point to note from the above data is that vials open to main refrigerator air flow returned to the specified temperature range the most quickly, while those contained in a thermally insulated container, such as a cardboard box or inside the vegetable crisper drawer, took the longest to recover to an allowed temperature. The dual-zone refrigerator required approximately $12 \mathrm{~h}$ to completely re-equilibrate from ambient temperature following the simulated power outage in trial with water bottles in place, and $9 \mathrm{~h}$ in the trial without water bottles. 


\subsubsection{Defrost Cycle}

Throughout the course of the dual-zone refrigerator measurements, we noted the occurrence of periodic temperature spikes due to the refrigerator's defrost cycle. The defrost cycle appears to run at $28 \mathrm{~h}$ to $34 \mathrm{~h}$ intervals. As evidenced by the graph below (Fig. 13), which shows a dual-zone refrigerator model defrost cycle, vaccine vial temperatures were not greatly affected by the periodic temperature increases.

During the cycle shown, one of the vial-attached thermometers (13) measured temperatures above the $8{ }^{\circ} \mathrm{C}$ temperature limit for a period of approximately $10 \mathrm{~min}$. However, in the majority of the defrost cycles we observed, all of the monitored vials remained within $2{ }^{\circ} \mathrm{C}$ to $8{ }^{\circ} \mathrm{C}$. The dual-zone refrigerator's defrost cycle is likely to have minimal effect on drug potency, but this will depend on the manufacturer specifications for the number of hours the vaccine can be held at certain temperatures greater than $8{ }^{\circ} \mathrm{C}$ before suffering a loss of viability.
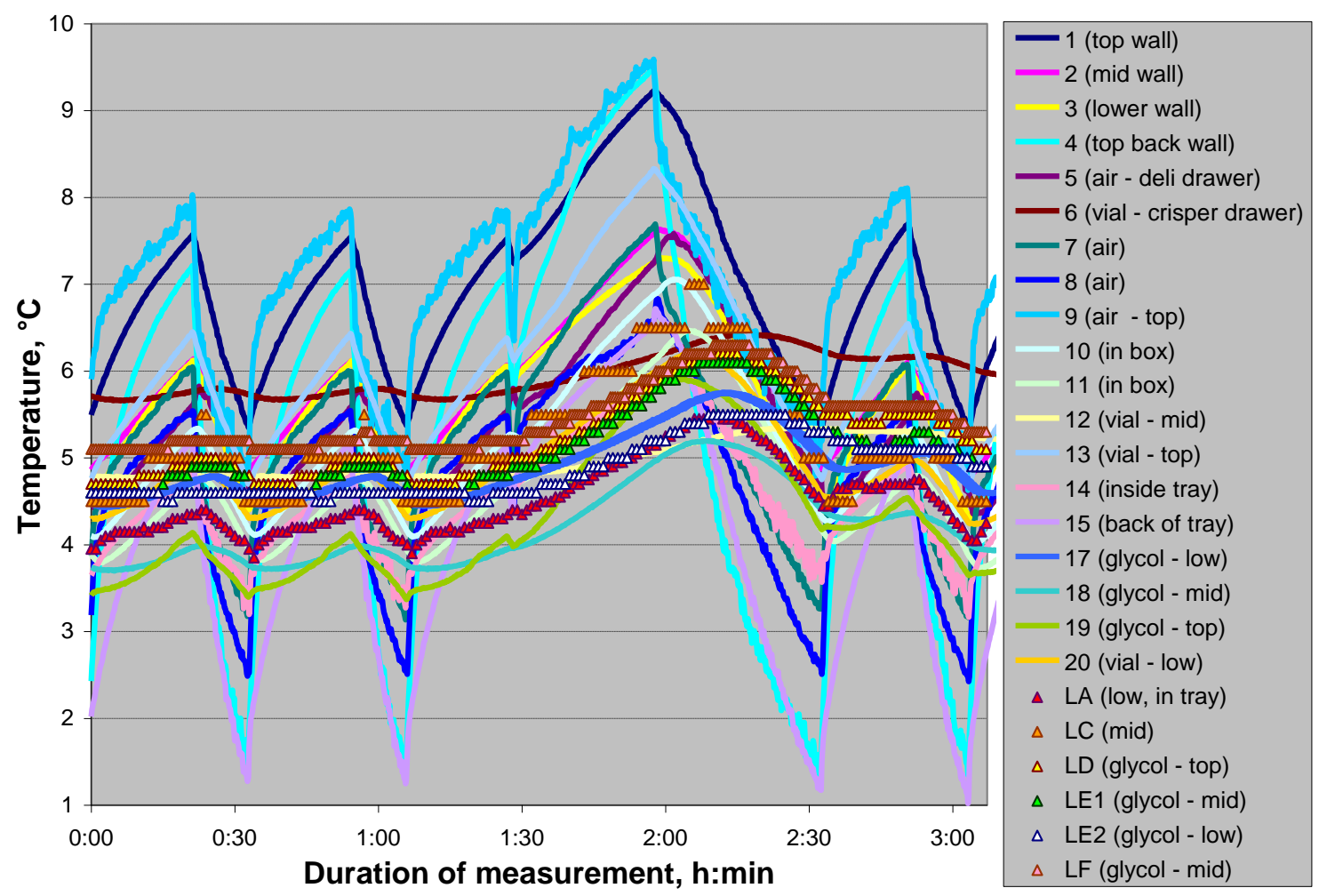

Fig. 13. Temperature response during a defrost cycle.

However, depending on the placement of thermometers used to monitor vaccine temperatures, the incidence of a defrost cycle could cause a VFC worker to incorrectly surmise that vaccine temperatures strayed outside of both the allowed maximum and minimum temperature bounds. Referring again to the graph, some of the thermometers attached to walls (e.g. 1,4), in close proximity to a wall (15), or hanging in the air (e.g. 7, 9) recorded large temperature spikes followed by a drop below the $2{ }^{\circ} \mathrm{C}$ limit. If one of 
these thermometers were used as an indicator of vial temperature, then employees may erroneously adjust the refrigerator set-point temperature and dispose of vaccines needlessly. As a result, care must be taken in determining the best placement for temperature monitoring devices in order to most accurately determine vial temperature during events such as refrigerator defrost cycles.

\subsubsection{General Stability of Vial Temperature and Suitability of Dual-Zone Refrigerator Model for Maintenance of Vaccine Storage Conditions}

The dual-zone refrigerator performance appears suitable for vaccine storage. Vaccine vial temperatures remained within the specified $2{ }^{\circ} \mathrm{C}$ to $8{ }^{\circ} \mathrm{C}$ range throughout all of the packing variation trials, as well as during the 15 second door opening trials. Drift of the refrigerator control thermometer appears to be negligible.

\subsubsection{Best and Worst Locations for Vial Storage}

Throughout the course of the closed-door dual-zone refrigerator trials, vaccine vials remained within the desired temperature range regardless of their location in the refrigerator. However, based on the results of the door opening and power-off trials, it appears that some storage methods and locations within the refrigerator provide better thermal stability than others.

In general, vials stored inside a thermally isolated container experienced less temperature disruption during normal, periodic door-opening operation than unpackaged vials kept in trays. Although existing vaccine storage and handling guidelines often advise against storing vaccines in the vegetable bins of a refrigerator, we found in our study that a vial stored in this area was subject to significantly less temperature variation than vials stored in the main body of the refrigerator. This is not surprising, because the crisper drawer acts as an additional barrier to reduce the rate and amount of heat transferred to the vials inside. While the unpackaged vials stored in trays experienced small temperature spikes each time the refrigerator door was opened, the temperature of the vial in the vegetable bin (TC \#6) was largely unaffected. During both the door left ajar tests and power outage trials, vial number 6 was one of the last monitored vials to exceed the $8{ }^{\circ} \mathrm{C}$ threshold.

If a provider chooses to store vaccines in the thermally isolated vegetable drawer, they should be aware of two possible caveats. First, while our test refrigerator did not have this feature, some refrigerator models include vegetable drawers with adjustable temperature or humidity controls. In this case, the provider should monitor the temperature inside the drawers, adjusting the controls as necessary until the required equilibrium conditions are met. Secondly, during our testing of the dual-zone refrigerator, we saw that the average temperature of the vegetable bin vial was generally about $1{ }^{\circ} \mathrm{C}$ warmer than that of the vials stored in the main refrigerator space. In our tests, this temperature difference was not enough to push the vegetable bin vial out of range. However, because the bins create a thermally isolated environment, workers cannot just assume that the temperature is the same inside and outside of the drawer. Again, any 
possible issues can easily be detected and remedied by simply monitoring the temperature inside the bin to insure that proper vaccine storage conditions are maintained.

Vials stored in cardboard boxes also experienced greater temperature stability than those that were left unpackaged. Like the vegetable drawers, the extra layer of cardboard reduced heat transfer to the vials inside.

During the simulated power outage trials, the vials placed in the bottom portion of the refrigerator were the last to exceed the allowed maximum temperature. During normal operation, fans inside the refrigerator distribute warmer and cooler air to maintain a fairly uniform temperature distribution throughout the interior space. Once a power outage begins, pockets of warmer and cooler air begin to separate, as the cooler air settles around the floor of the refrigerator and the warm air rises towards the top. As a result, vials kept on the upper shelves and not contained in boxes experienced the fastest temperature increases.

Throughout the range of tests performed, one thermocouple inside a glycol-filled bottle was kept on the top refrigerator shelf, underneath the cooling vent (TC \#19). This thermocouple consistently recorded temperatures below the $2{ }^{\circ} \mathrm{C}$ threshold, and did not show temperature behavior consistent with the other sensors in the refrigerator. Because the glass bottle filled with liquid glycol approximates the thermal mass and properties of a vaccine vial, a vial placed in the same location would likely experience similar results. The proximity of the cooling vent above this location means that every time the refrigerator compressor cycles on, the stream of cold air drives the temperature downward. The dual-zone refrigerator model we tested featured glass shelves, which have more surface area and a greater heat capacity than the wire shelves in some other models. It takes more heat to change the temperature of the glass a certain amount than it does to change the surrounding air by the same degree. The result is that the temperature of the glass shelf located underneath the cooling vent stays colder than some other areas of the refrigerator. In our study, the glycol-filled bottle in direct contact with this shelf experienced temperatures an average of $3{ }^{\circ} \mathrm{C}$ to $5{ }^{\circ} \mathrm{C}$ colder than the rest of the refrigerator.

Another thermocouple inside a glycol-filled bottle (TC \#18) was placed one level down, directly on the middle refrigerator shelf. This thermocouple consistently recorded temperatures at least $0.5{ }^{\circ} \mathrm{C}$ colder than any of the glycol bottle or vial-attached sensors stored in trays or boxes. A maintenance temperature difference of $0.5{ }^{\circ} \mathrm{C}$ is not likely to cause thermal excursions unless there are existing issues with the refrigerator temperature set point adjustment or control. However, a $5{ }^{\circ} \mathrm{C}$ temperature difference is very likely to result in an out of range condition. Most providers do not have the equipment, time, or incentive to study the complete temperature distribution of their vaccine refrigerators, and as a result, they are not likely to know which shelves and areas correspond to hazardous "cold spots." Because the potential for damage is high, providers should not store vaccines in direct contact with the surface of any glass shelf, regardless of its position inside the refrigerator. In addition, trays or boxes containing vaccine vials should not be placed directly under the cooling vent. 


\subsubsection{Best and Worst Methods of Monitoring Vial Temperature}

In this study, we used several different temperature sensing devices in various locations throughout the refrigerator in order to determine the best way to monitor actual vial temperature. While attaching a thermocouple thermometer to a vial is a very accurate way to determine vaccine temperature, this is not a feasible strategy for clinics or storage facilities. To determine the suitability of different vial temperature monitoring schemes, that is, the best and worst locations and monitoring devices for this purpose, we can compare the temperature readings of the vial-attached thermocouples to the readings of the other thermometers. 

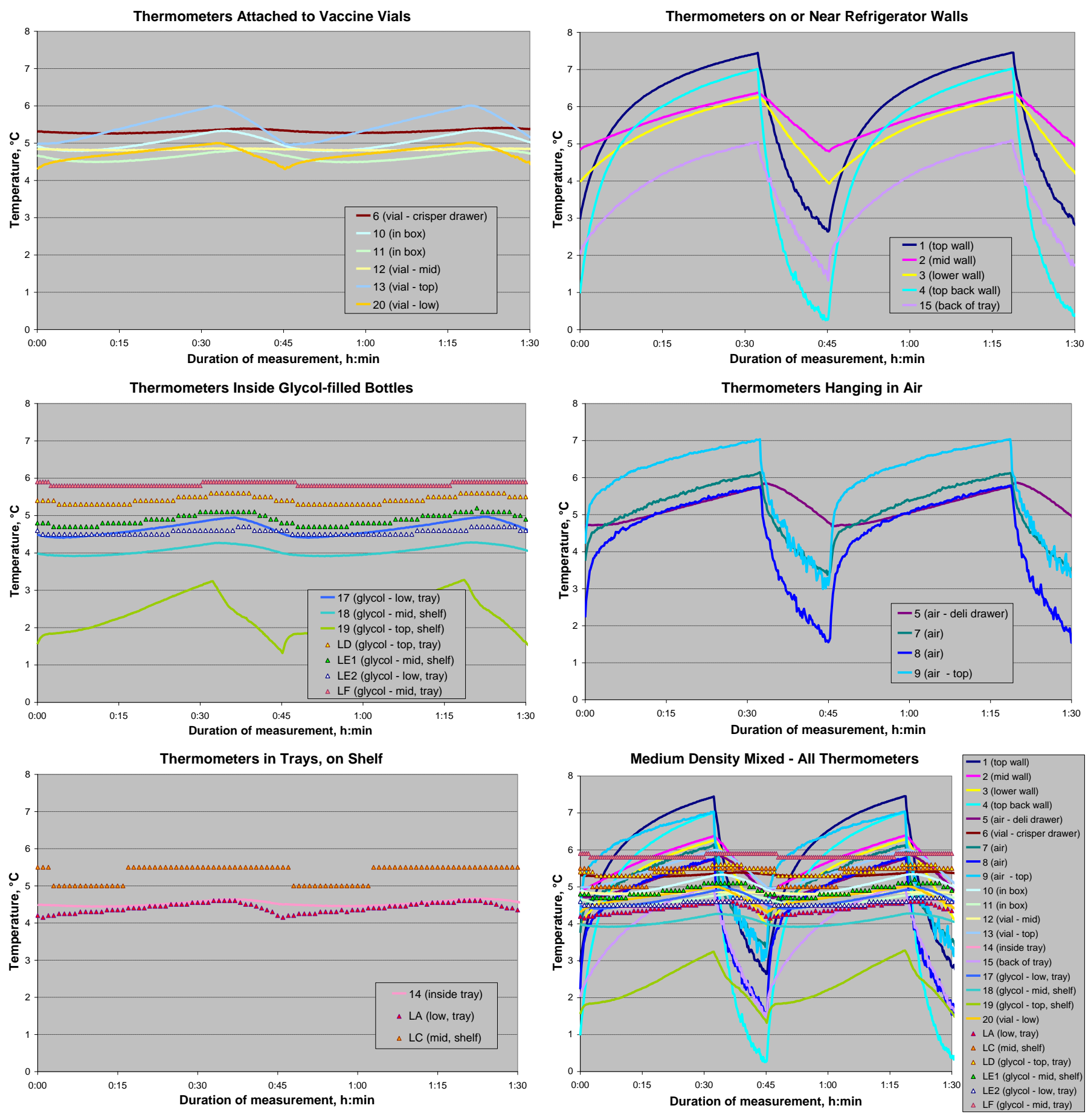

Fig. 14. Response of thermometers with different mounting methods. 


\section{Best Locations for Temperature Sensors}

- A thermocouple contained inside a glycol filled bottle placed in a plastic tray matched the vaccine temperatures very closely (TC \#17). During the door-opening and poweroff trials, this thermometer displayed a temperature response similar to nearby the vial thermometers.

- However, two other sensors in glycol filled bottles (TC \#s 18, 19) gave very different results, as they were both placed directly on upper refrigerator shelves, in contact with the glass and in close proximity to the cooling vent.

- A thermocouple kept inside a glycol filled bottle provides a good approximation for the thermal mass of a glass vaccine vial. However, if the bottle is placed in an area that is not representative of the main body refrigerator temperature, the readings will not give a good indication of vial temperature. The glass shelves directly under the cooling vent stayed at a colder temperature than the rest of the refrigerator, skewing the readings of TC \#18 and 19, which were in direct contact with these shelves. By keeping TC \#17 in a tray on a lower shelf instead of directly on the glass, this effect was mitigated.

- Loggers C, D, E1, and E2 agreed closely with the temperatures recorded by the vialattached thermocouples.

- Loggers D, E1, and E2 each had a probe inside a glycol-filled bottle and was placed in a tray, closely replicating the thermal conditions of stored vaccine vials. Throughout the range of testing, the temperature variation of vials stored in different locations stayed within $1.5^{\circ} \mathrm{C}$. The readings of each of the loggers with probes in glycol bottles (LD, LE1, LE2) consistently stayed within the same temperature span as the vials. As such, temperature probes kept inside glycol-filled bottles appear to give a very good representation of vaccine vial temperature, provided they are not placed in an area with "outlier" conditions, such as directly under the cooling vent.

- As a general rule, the closer the thermometer is placed to the actual location where vaccines are stored, the closer its readings will be to actual vaccine temperatures.

\section{Mediocre Locations for Temperature Sensors}

- Thermometers placed on main refrigerator shelves or inside of a tray in the areas where vaccines were stored produced data reasonably similar to that of the vial thermometers most of the time (TC \#s 14, 18; LA, LF).

- Thermocouple 14 provided reasonable thermal mapping of recorded vial temperatures, although tended to average about $0.5^{\circ} \mathrm{C}$ colder than the majority of the vial attached thermocouples.

- On average, thermocouple 18 measured $0.5^{\circ} \mathrm{C}$ to $1{ }^{\circ} \mathrm{C}$ colder than vial temperatures. Perhaps because TC \#18 was in direct contact with the glass shelf, one level down from the cooling unit. Placing temperature monitors directly on glass shelves should be avoided. Instead, the monitors should be kept in the same types of trays in which vaccines are stored.

- Logger A (LA) showed temperature behavior similar to vial 20, as both were stored in trays in the lower portion of the refrigerator. Even so, the average temperatures recorded by LA were $\sim 0.5{ }^{\circ} \mathrm{C}$ colder than those measured on vial 20. By contrast, logger $\mathrm{F}$ consistently recorded temperatures $0.5^{\circ} \mathrm{C}$ to $2{ }^{\circ} \mathrm{C}$ warmer than vials stored nearby. Both loggers were kept in a plastic tray. 
- One thermocouple (TC \#5) was hung from inside the refrigerator deli drawer. This somewhat thermally isolated environment prevented the thermometer from reading the dramatic temperature fluctuations recorded by thermometers hanging in the main airspace of the refrigerator. However, during the door opening trial, this thermometer recorded much larger temperature spikes than any of the thermometers attached to vaccine vials. As a result, the interior of the refrigerator deli drawer is not an ideal location to store temperature monitors.

\section{Unacceptable Locations for Temperature Sensors}

- Thermometers attached to refrigerator walls (TC \#s 1, 2, 3, 4) registered temperatures that differed greatly from vial temperatures. These thermometers measured dramatic temperature fluctuations in response to normal refrigerator temperature control and defrost cycles as well as during our simulation trials. Thermometers 1 and 4 were closest to the cooling unit, so they recorded very large temperature changes $\left(5^{\circ} \mathrm{C}\right.$ to 7 ${ }^{\circ} \mathrm{C}$ ) in response to the standard refrigerator cooling cycle. This also caused TC \#1 to repeatedly drop below $2{ }^{\circ} \mathrm{C}$.

- TC \#15, attached to the back of a tray, showed large temperature fluctuations similar to the wall-attached thermocouples, most likely due to its proximity to the wall.

- A thermometer set up to record air temperature is also not a good indicator of vial temperature. Thermometers hanging in the air of the main refrigerator cavity (TC \#s $7,8,9)$ showed large temperature fluctuations in response to the refrigerator cooling cycle. Thermometer 8 repeatedly showed a dip below $2{ }^{\circ} \mathrm{C}$ during closed-door trials. During door opening trials, all three thermometers hanging in air showed large temperature spikes exceeding $8{ }^{\circ} \mathrm{C}$.

- The thermal mass of a glass vaccine vial reduces its temperature sensitivity, so that it takes the vial longer to heat up or cool down in response to air temperature changes. Relying on air temperature as an indicator of vaccine storage conditions is likely to give false alarms, leading workers to believe a thermal excursion has occurred when in fact vaccine temperatures remained within range.

- Temperature monitors should never be placed directly under the cooling vent, as the temperature in this area will be much colder than vaccines stored elsewhere in the refrigerator. TC \#19, on a shelf directly under the vent recorded temperatures an average of $3{ }^{\circ} \mathrm{C}$ to $5{ }^{\circ} \mathrm{C}$ colder than the rest of the refrigerator.

\subsubsection{Dual-Zone Refrigerator Case Study: Does freezer setting affect refrigerator performance?}

While completing the standard measurement matrix using the dual-zone refrigerator, we considered only the refrigerator compartment of the unit. The freezer compartment was kept empty for the duration of the study, and its temperature control settings were left unchanged. The preceding results from the dual-zone model tests address the unit's suitability as a refrigerated vaccine storage unit only. Although the dual-zone model we tested featured separate refrigerator and freezer temperature controls, it is possible that changing the freezer temperature settings or adding contents to the freezer compartment could impact the temperature of the unit's refrigerator compartment. VFC providers and 
program managers have given anecdotal reports of dual-zone refrigerator chambers becoming excessively cold and freezing refrigerated vaccine supplies when the freezer temperature controls are adjusted to properly maintain frozen vaccines. An in-depth evaluation of the performance of dual-zone units used to store refrigerated and frozen vaccine simultaneously was outside the scope of our study. However, we performed a brief case study aimed at addressing some of the questions about synchronized refrigerator and freezer operation.

In this case study, we performed three trials using a medium-density, mixed container loading pattern in both the refrigerator and freezer compartments. We used the same 19 thermocouples and 6 data electronic loggers as in the refrigerator-only testing. The temperature sensors were arranged throughout the refrigerator and freezer chambers, attached to walls, inside trays, with probes inside glycol-filled bottles, hanging in the air, and attached to vaccine vials. Thermocouples 1 through 10 were installed in the freezer compartment along with data loggers LC and LE2. Thermocouples 11 through 20, and data loggers LA, LD, LE1 and LF were placed in the refrigerator chamber. The labeled installation pattern is shown in figure 15 below.

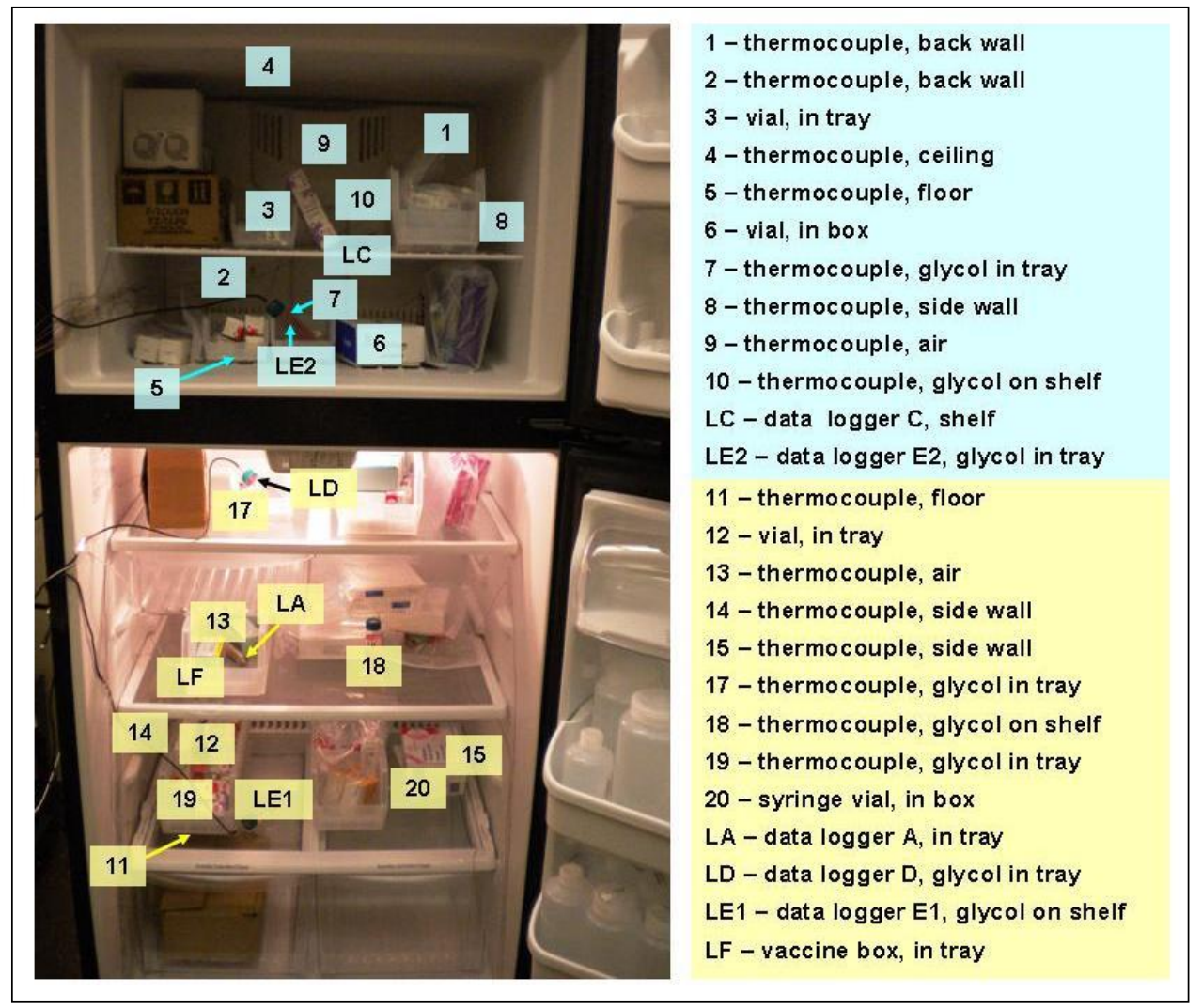

Fig. 15. Thermocouple and data logger installation pattern-dual-zone refrigerator/freezer test.

Using this installation pattern, we performed three trials, varying the freezer temperature control setting while leaving the refrigerator temperature control unchanged. For the first trial, the freezer temperature control dial was set at the midpoint, halfway between the 
minimum and maximum dial settings, the same setting used throughout our standard refrigerator-only testing procedures. During the second trial, the freezer temperature control dial was adjusted to $75 \%$ of the maximum cold setting. For the third and final trial, the dial was turned $100 \%$ of the way to the "coldest" temperature setting. Measurements for each trial were conducted in the same fashion as discussed in the closed-door refrigerator tests, collecting data overnight.

The average temperature readings for each of the thermometers across the three trials are shown in figure 16 below. Thermometer temperature averages were taken over a $2.5 \mathrm{~h}$ representative sample of data from each trial. Data from the freezer sensors and refrigerator sensors are displayed in two equally-scaled graphs, for ease of comparison.
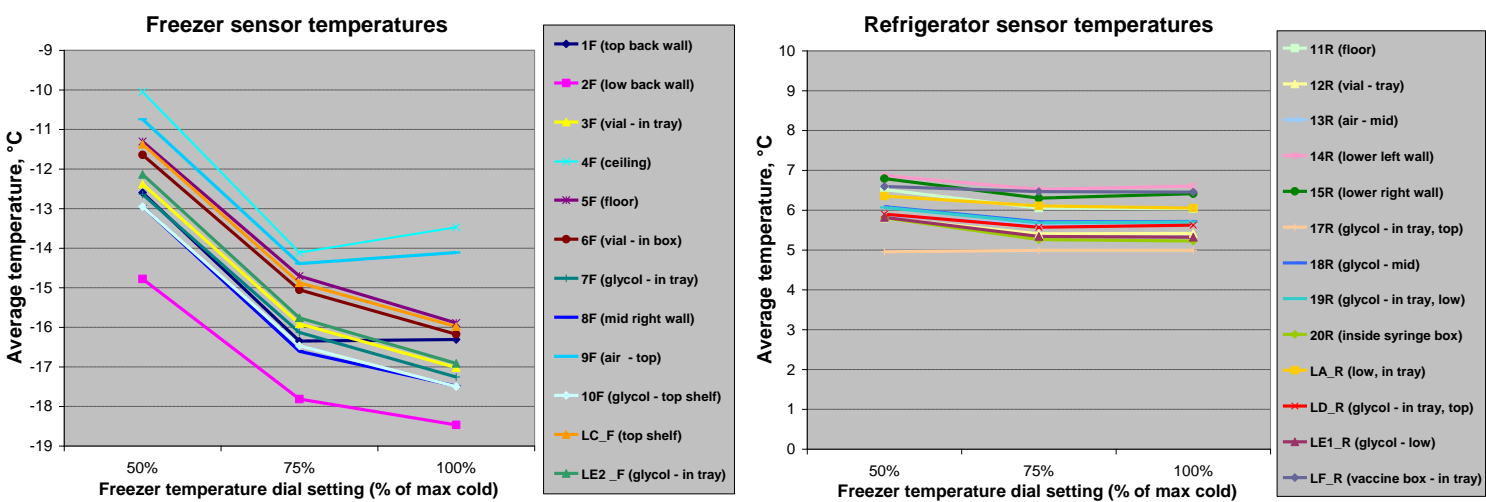

Fig. 16. Average temperatures recorded with freezer temperature dial set to $50 \%, 75 \%$, and $100 \%$ of maximum cold setting. Data from thermometers inside the freezer compartment is shown on the left; refrigerator thermometer data is on the right.

As expected, the majority of the freezer sensors recorded colder temperatures when the freezer set point was adjusted to lower temperature settings. Comparing the subsequent temperature changes of the refrigerator sensors, we can see that certain locations inside the refrigerator did record a temperature drop in response to the freezer set point adjustment. However, the magnitude of the drop was much smaller than inside the freezer compartment. The relationship between freezer temperature change and its impact on the refrigeration chamber is only about 10:1, meaning that in order for the refrigerator temperature to drop by $1{ }^{\circ} \mathrm{C}$, the freezer must be adjusted a full $10{ }^{\circ} \mathrm{C}$ colder. In our brief testing, we "maxed-out" the freezer's cooling capabilities by setting it to its absolute coldest temperature. In the graphs above, we can see that even at the maximum coldest setting, the interior refrigerator temperature was not significantly impacted, and none the sensor temperatures dropped much below $5{ }^{\circ} \mathrm{C}$.

While the results of this brief investigation should not be taken as a hard and fast rule to all dual-zone refrigeration systems, in the case of our unit, it appears that merely adjusting the freezer set point control is unlikely to result in major changes in the refrigerator compartment temperature, much less cause vaccines to drop below $0{ }^{\circ} \mathrm{C}$. However, in the case of a dual-zone unit with only one temperature control dial for both refrigerator and freezer compartments, it is much more likely that attempts to lower freezer temperature could result in an undesirably low refrigerator temperature. For this 
reason, attempting to store both frozen and refrigerated vaccines in a unit with only one temperature set point control is not a recommended practice.

Although our results seem to indicate that the freezer temperature in a dual-control dualzone unit can be adjusted without negatively impacting refrigerated vaccine storage, perhaps a more important question is whether this type of refrigerator-freezer unit is suitable for storing frozen vaccines in the first place. The two graphs in figures 17a and $17 \mathrm{~b}$ below show samples of data collected during the first and third case study trials, in which the freezer temperature was set to $50 \%$ and $100 \%$ of the maximum cold output, respectively.
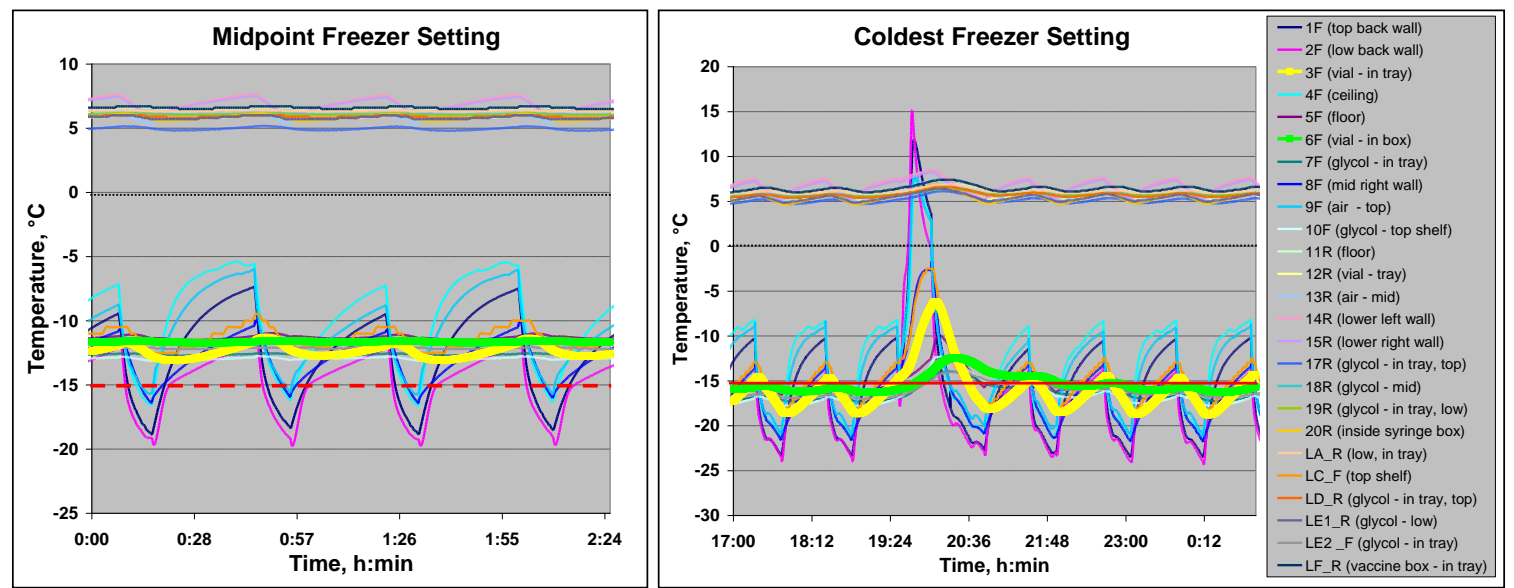

Fig. 17a. (Left) Temperature data collected during midpoint freezer setting trial.

Fig. 17b. (Right) Temperature data collected during maximum cold freezer setting trial. Temperature spike due to defrost cycle is also shown.

The required storage temperature for most frozen vaccines is $<-15^{\circ} \mathrm{C}$. This temperature is indicated by a red line on each of the graphs. The two monitored vials kept in the freezer are shown in bold green and yellow (thermocouples 3 and 6). When the freezer was set to the midpoint temperature, vial temperatures stayed out of range for the duration of the trial, with average temperature readings of approximately $-12{ }^{\circ} \mathrm{C}$. Even in the second graph, which shows data taken at the maximum cold setting, the two monitored vials consistently show temperatures above $-15{ }^{\circ} \mathrm{C}$ at the peak of each compressor cycle, fluctuating between $-19{ }^{\circ} \mathrm{C}$ and $-13{ }^{\circ} \mathrm{C}$. Even with the control dial set to its coldest temperature, the freezer does not appear to maintain suitable frozen vaccine storage temperatures. Furthermore, the normal temperature fluctuations associated with freezer control (up to $5{ }^{\circ} \mathrm{C}$ ) are much larger than the corresponding fluctuations in the refrigerator compartment. This means that there is very little room for temperature set point error. Perhaps even more troubling is the temperature spike associated with the defrost cycle, which can be seen in the second graph. During the defrost cycle, one of the vials recorded temperatures as high as $-6^{\circ} \mathrm{C}$, a major excursion above the specified $-15^{\circ} \mathrm{C}$ maximum. Depending on the vaccine manufacturer's storage specifications, repeated exposures to these types of temperature spikes could cause frozen vaccines to suffer a loss of viability. Coupled with the fact that this refrigerator-freezer unit simply does not appear capable of maintaining $-15^{\circ} \mathrm{C}$ freezer temperatures, users might question whether 
the dual-zone units are suitable for any frozen vaccine storage. Because our investigation into this issue was limited, we do not intend to completely rule out all dual-zone units for frozen vaccine storage. However, users should take extreme caution when relying on these types of units for maintaining correct freezing temperatures. Before attempting to store vaccines in this type of freezer, users should continuously monitor freezer temperature for several days to determine whether the unit consistently maintains an acceptable set point temperature. The thermometer used should be placed in the same location where vaccines will be stored. During the preliminary freezer evaluation, users should also examine whether defrost cycle effects will put stored vaccines at risk. If the freezer compartment is deemed suitable for vaccine storage after careful analysis of the preliminary data, users may cautiously proceed with loading vaccines. However, they should keep their continuous temperature monitoring system in place and frequently reevaluate new data to verify that the proper storage conditions are maintained.

The overall performance of the dual-zone freezer compartment during our testing was not suitable for vaccine storage. While adjusting the freezer set point did not appear to negatively impact storage temperatures inside the refrigerator chamber, the unit did not maintain the desired freezer temperatures, even at its maximum coldest setting. If possible, frozen vaccines should be stored in a standalone, freezer-only unit in order to avoid the issues we encountered during this case study.

\subsection{Pharmaceutical Refrigerator}

\subsubsection{Effect of loading density}

In trials performed using this refrigerator model, vaccine storage density did not appear to have a major impact on the refrigerator's capability to maintain the desired temperature range. The photographs below (Fig. 18) show the various levels of packing density that were tested.

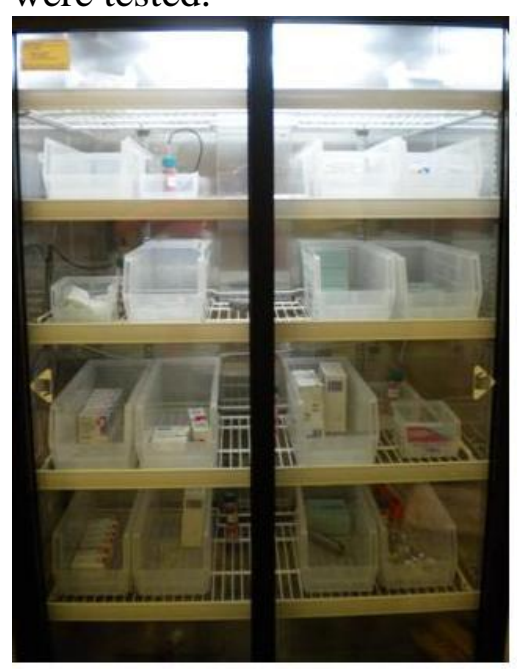

Low Density Pack

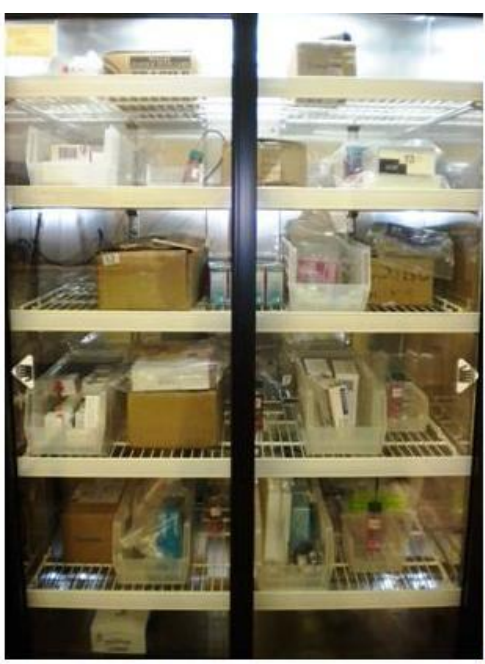

Medium Density Pack

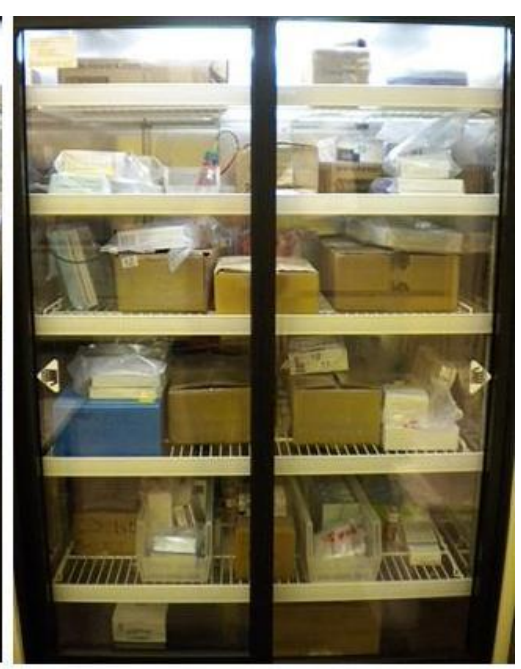

High Density Pack

Fig. 18. Typical packing patterns. 
Throughout the course of the study, average thermometer temperatures remained within approximately a $2{ }^{\circ} \mathrm{C}$ range regardless of whether the refrigerator load was low, medium, or high density, as seen in figure 19. All of the thermometers maintained average temperatures within the prescribed $2{ }^{\circ} \mathrm{C}$ to $8{ }^{\circ} \mathrm{C}$ range, with the exception of two thermocouples ( 2 and 3 ), which were both taped to the interior left wall of the unit. Both of these thermometers were fairly close to the access port which was used to connect all of the sensor leads to the data collection system outside the refrigerator. This may have been a factor if an appreciable amount of heat leaked through the port. It is also possible that the nature of the cooling system and vents in this particular refrigerator model simply do not cool the side refrigerator walls to same temperature as the back walls or the rest of the refrigerator interior. Thermocouple 4, taped to the top back wall, and thermocouple 9 , hanging from the refrigerator ceiling, also both recorded thermal excursions not experienced by thermometers placed in other areas of the refrigerator. Thermocouples 4 and 9 maintained average temperatures within the prescribed $2{ }^{\circ} \mathrm{C}$ to $8{ }^{\circ} \mathrm{C}$ range throughout the duration of testing. However, these thermocouples routinely recorded point measurements below $2{ }^{\circ} \mathrm{C}$ in response to the refrigerator's cyclic temperature control pattern.

In general, outlier temperature behavior in the pharmaceutical refrigerator seems to be correlated to placement of sensors in direct contact with the refrigerator walls or near the top cooling vent. Relying on a thermometer placed in one of these areas to adjust the refrigerator set point could cause users to mistakenly set the refrigerator temperature too warm or too cold. As a result, temperature monitors should be placed in the main body of the refrigerator, away from walls and top cooling vent. In addition, users should not place vaccines in direct contact with the walls, a practice that is excluded by both manufacturer recommendations as well as $\mathrm{CDC}$ vaccine storage and handling guidelines. If these conditions are met, out of range wall temperatures will not negatively affect stored vaccines. 


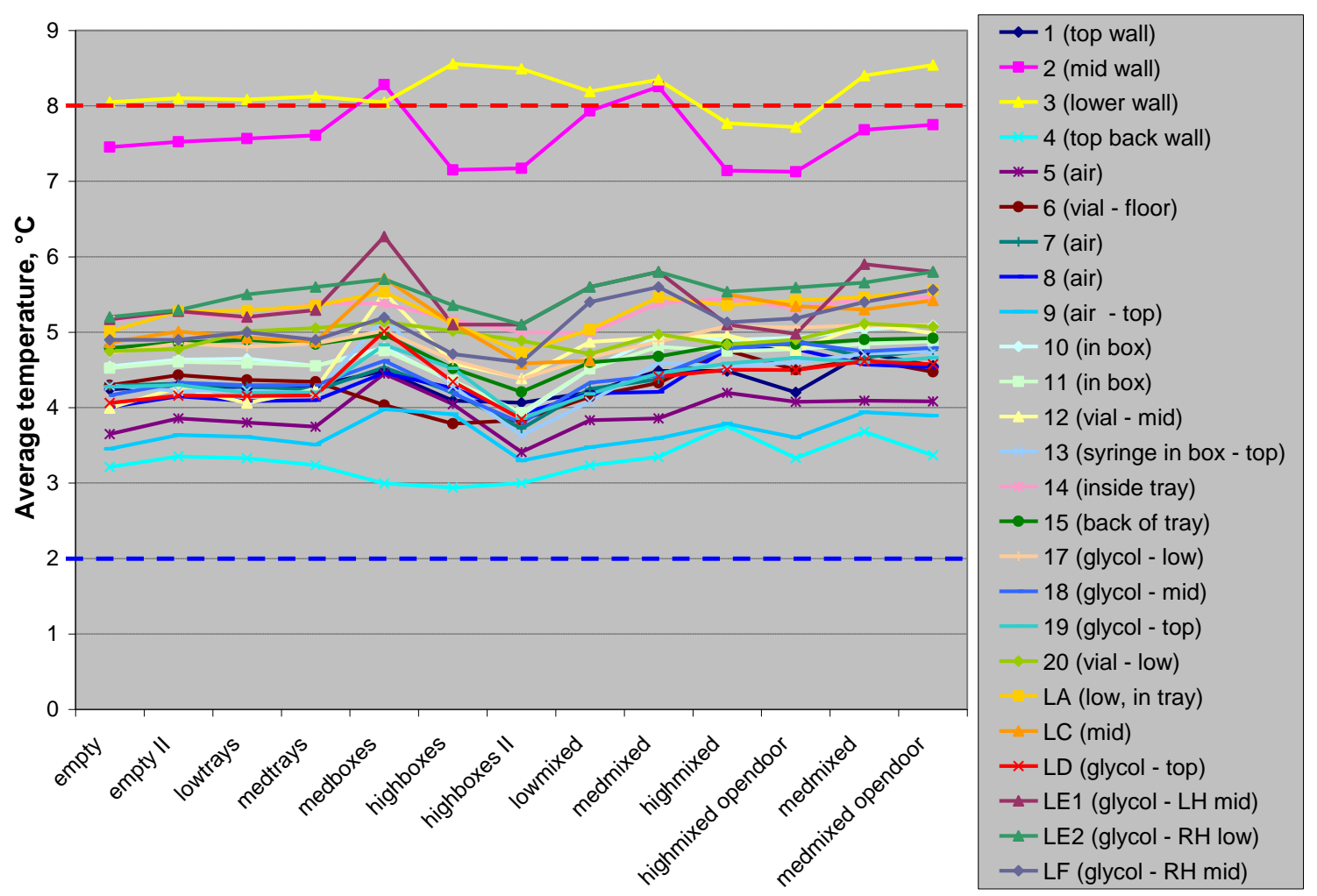

Fig. 19. Average temperature of pharmaceutical refrigerator for all sensors, as a function of loading pattern. Labels on the abscissa correspond to entries in the measurement matrix of Table 1 .

Figure 19 shows the average reading for each thermometer across all of the closed-door pharmaceutical refrigerator trials, as well as the periodic door-opening trials. Other than the two wall-attached thermocouples discussed previously, it is readily apparent that the main body of the refrigerator as well the vaccine vials themselves stayed within the prescribed temperature range, regardless of packing density variations. The temperature distribution of the remaining thermocouples, including all of those attached to vaccine vials, also remained consistent regardless of load density. Over the entire range of testing, a total of 45 days, the vertical shift of this distribution was less than one degree. This demonstrates that the pharmaceutical refrigerator maintains stable set point control across variable packing densities and schemes that are likely to occur in a vaccine storage system. As a result, the pharmaceutical refrigerator model appears to be more than sufficient for normal vaccine storage.

\subsubsection{Effect of packing style}

As with the loading density variation, changing the vaccine packing style had little to no impact on the temperature stability of the refrigerator, whether plastic trays, cardboard boxes, or a combination of both were used to store the vaccines. (See Fig. 20.) The measurement results indicate that the pharmaceutical refrigerator model successfully maintains proper vaccine storage temperatures range regardless of packaging style. 


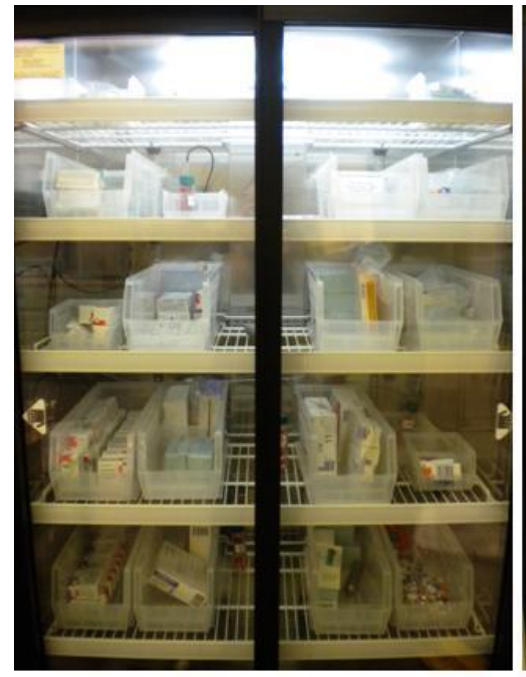

Plastic Trays

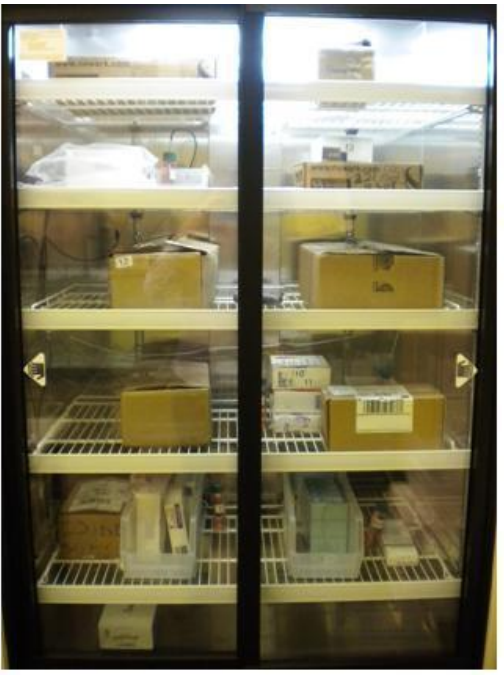

Cardboard Boxes

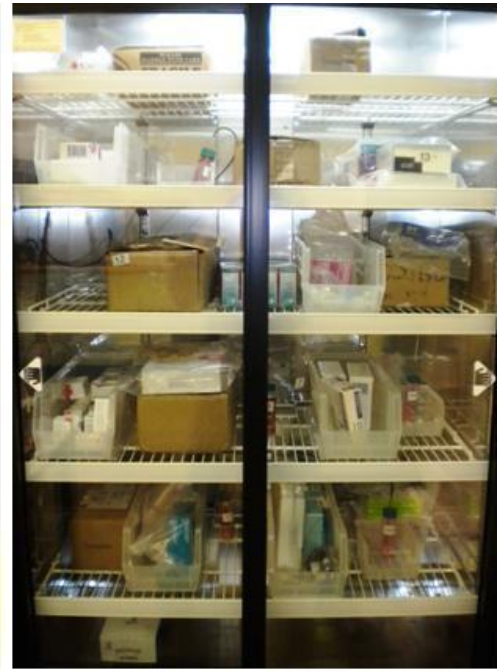

Mixed Trays and Boxes

Fig. 20. Various vaccine storage methods.

\subsubsection{Effect of door opening and closing —normal use simulation}

The results of the periodic door-opening trials also support the efficacy of the pharmaceutical refrigerator model. The only thermocouples which were subjected to thermal excursions in response to the door opening were those that were hanging in the air (e.g. TC \#s 7, 9) or attached to the interior refrigerator walls (e.g. TC \#s 2,3). We already saw that several of the wall-attached thermocouples consistently recorded out of range temperatures during the closed-door tests. The only significant impact of periodically opening the refrigerator door was to cause brief spikes in the refrigerator air temperature. All of the remaining thermocouples, whether attached to vials or syringes, kept inside glycol-filled bottles or in plastic trays, as well as each of the electronic data loggers continued to record temperatures within the specific $2{ }^{\circ} \mathrm{C}$ to $8{ }^{\circ} \mathrm{C}$ range throughout the door-opening trials. 


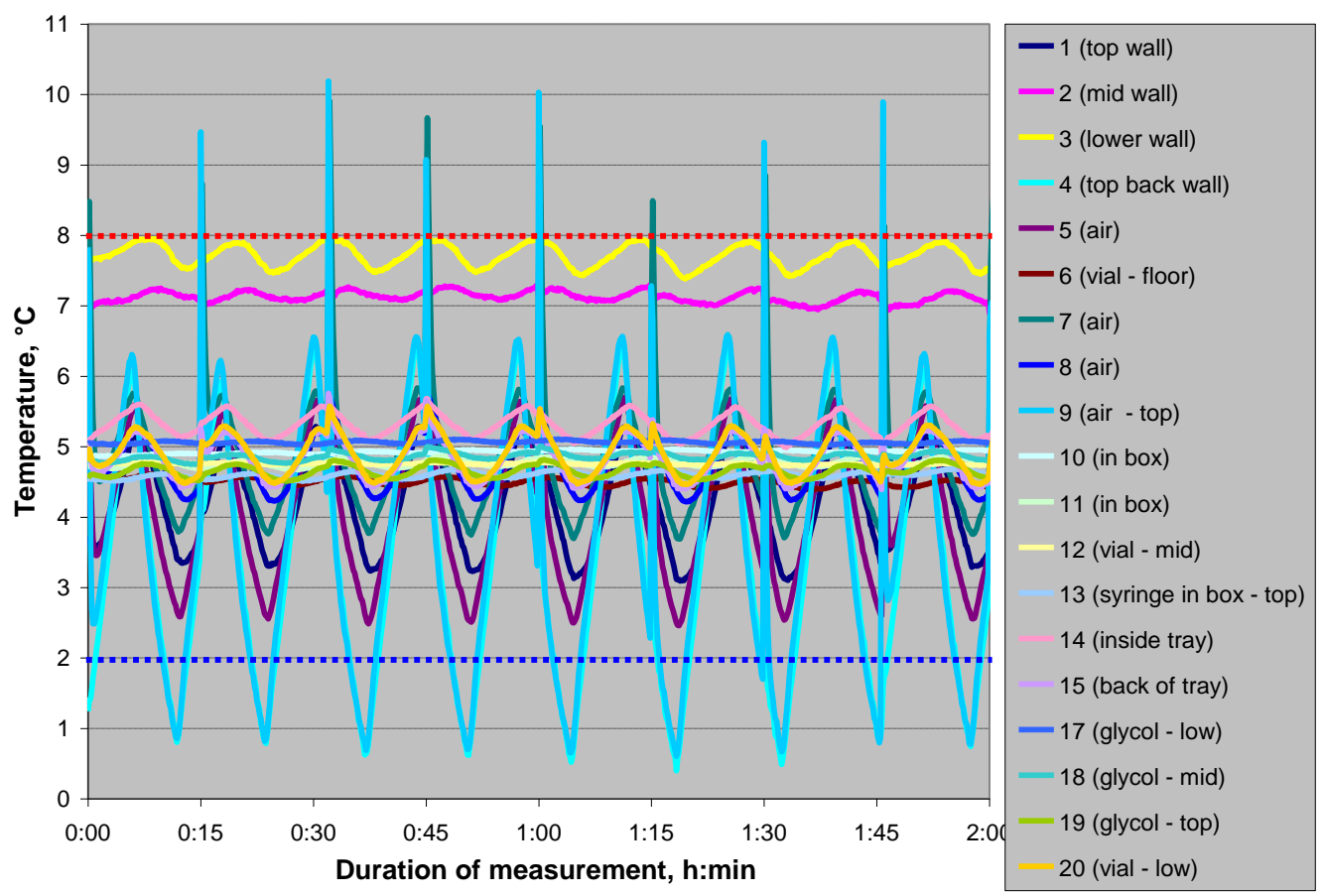

Fig. 21. Door opening trial for the pharmaceutical refrigerator, using a high density mixed load.

In figure 21 above, two thermocouples (7 and 9), both hanging in the air, show temperature spikes above $8{ }^{\circ} \mathrm{C}$ each time the refrigerator door is opened. Both thermocouples 9 and 4 also continually drop below $2{ }^{\circ} \mathrm{C}$ in response to the refrigerator's cooling cycle. However, this effect is present regardless of whether the refrigerator door is opened or kept closed, and as we can see from the remaining thermocouple data, does not impact the refrigerator's ability to keep stored items within the specified temperature range. All monitored vaccine vials and thermocouples inside glycol-filled bottles were maintained at temperatures around $5{ }^{\circ} \mathrm{C}$ in spite of repeated exposure to room temperature air. Figure 22 below shows the data recorded by these sensors only.
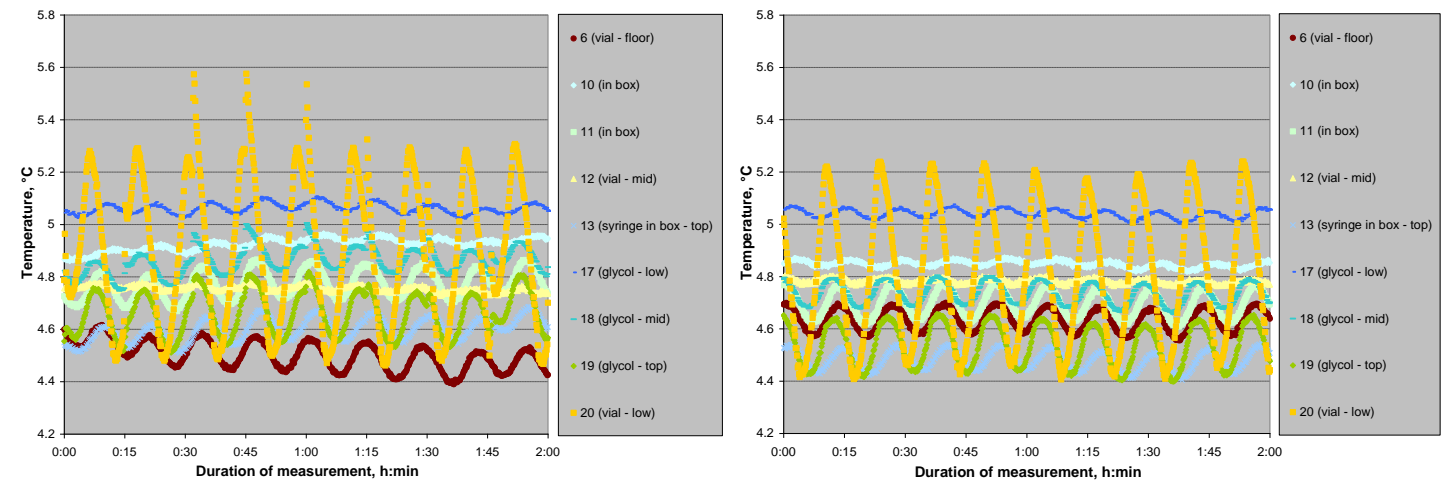

Fig. 22a. (Left) Door opening trial for the pharmaceutical refrigerator. Only thermocouples monitoring vial temperatures or inside glycol-filled bottles are shown.

Fig. 22b. (Right) Temperature response of same selected thermocouples, recorded $24 \mathrm{~h}$ later and with refrigerator door kept closed. 
Figure 22a shows the selected thermometer responses to repeated door opening. Figure $22 \mathrm{~b}$ shows the closed-door, equilibrium temperature behavior of the same thermometers. The vertical scale of each of the graphs is only $1.6^{\circ} \mathrm{C}$. From this, it is clear that the pharmaceutical refrigerator preserves excellent temperature stability during normal use patterns. The net effect of opening the refrigerator door on stored vaccine vial temperature is extremely small. Only vial number 20, which was kept in an open tray rather than contained in a box or its original packing, showed any noticeable temperature spike each time the door was opened. Even then, this temperature spike was less than $0.5^{\circ} \mathrm{C}$. Several other vials show either a gradual temperature increase or decrease over the course of the door opening trial, depending on their location in the refrigerator. However, these changes are extremely small, on the order of $0.2^{\circ} \mathrm{C}$ or less. As the pharmaceutical refrigerator has already proven to possess excellent temperature set point control, it is highly unlikely that such miniscule temperature changes cause damage to stored vaccine.

The table below shows the different door opening temperature changes of all the thermometers in the high and medium density load trials. In this case, the temperature change is defined as the difference between the average temperature recorded during the closed door trial and the average temperature from the door-opening trial with the same loading configuration. No trials using water bottles as a temperature ballast were performed with the pharmaceutical refrigerator. In tests of the other refrigerator models, we filled the refrigerator doors with water bottles. Because the pharmaceutical model we tested had sliding glass doors which could not be used to store water bottles, we chose not to perform any trials using water bottles in order to keep our measurement methods consistent. It would be possible for users to place water bottles along the refrigerator walls or on the floor level to act as a thermal ballast. The purpose of a water bottle temperature ballast is to add extra thermal mass, thereby making the refrigerator less susceptible to temperature changes. However, throughout our tests of the pharmaceutical model, we saw that the unit was not significantly affected by varying load densities (Fig. 19). In practice, adding water bottles would likely produce an effect similar to adding a higher density vaccine load. Because the pharmaceutical model performed equally well under both high and low density packing schemes, the addition of water bottles would probably not have a major impact on refrigerator performance. Furthermore, the pharmaceutical refrigerator we tested featured air vents along the entire inner back wall of the unit. In this case, if users attempted to improve the system by placing water bottles along this wall, they might reduce air circulation and negatively impact refrigerator performance. 
Table 8. Temperature changes during door-opening tests (all values in degrees Celsius).

\begin{tabular}{|c|c|c|}
\hline Sensor Name/Location & High Density Mixed & Medium Density Mixed \\
\hline 1 (top wall) & -0.2842 & -0.1718 \\
\hline 2 (mid wall) & -0.0179 & 0.0667 \\
\hline 3 (lower wall) & -0.0526 & 0.1423 \\
\hline 4 (top back wall) & -0.4274 & -0.3139 \\
\hline 5 (air) & -0.1218 & -0.0091 \\
\hline 6 (vial - floor) & -0.2561 & -0.1656 \\
\hline 7 (air) & 0.0923 & 0.0957 \\
\hline 8 (air) & -0.0012 & -0.0371 \\
\hline 9 (air - top) & -0.1839 & -0.0462 \\
\hline 10 (in box) & 0.0282 & 0.0557 \\
\hline 11 (in box) & 0.0222 & 0.0356 \\
\hline 12 (vial - mid) & -0.2008 & -0.1203 \\
\hline 13 (syringe in box - top) & 0.0537 & 0.0330 \\
\hline 14 (inside tray) & -0.1264 & 0.0206 \\
\hline 15 (back of tray) & 0.0034 & 0.0198 \\
\hline 17 (glycol - low) & -0.0185 & 0.0017 \\
\hline 18 (glycol - mid) & 0.0789 & 0.0440 \\
\hline 19 (glycol - top) & 0.0766 & 0.0598 \\
\hline 20 (vial - low) & 0.0649 & -0.0401 \\
\hline LA (low, in tray) & 0.0665 & 0.0930 \\
\hline LC (mid) & -0.1563 & 0.1215 \\
\hline LD (glycol - top) & -0.0018 & -0.0407 \\
\hline LE1 (glycol - LH mid) & -0.1207 & -0.1000 \\
\hline LE2 (glycol - RH low) & 0.0545 & 0.1438 \\
\hline LF (glycol - RH mid) & 0.0517 & 0.1625 \\
\hline
\end{tabular}

Table 8 above provides further evidence that the pharmaceutical refrigerator model is largely unaffected by repeatedly opening and closing the refrigerator door. The majority of the sensors recorded a change of less than one tenth of a degree between the dooropening and closed door trials, and the largest change was still less than $0.5^{\circ} \mathrm{C}$.

Compared to other refrigerator models we tested, the pharmaceutical refrigerator resisted temperature change surprisingly well during trials in which the refrigerator door was left cracked open several inches for $1 \mathrm{~h}$, to simulate an employee failing to completely close the door. Table 9 below shows the amount of time elapsed once the door was opened until the monitored vials exceeded the allowed maximum temperature of $8{ }^{\circ} \mathrm{C}$.

Table 9. Time after opening door until vial temperature exceeded $8{ }^{\circ} \mathrm{C}$.

\begin{tabular}{|l|c|c|}
\hline \multicolumn{1}{|c|}{ Name and Location } & $\begin{array}{c}\text { High Density Mixed } \\
\text { (minutes) }\end{array}$ & $\begin{array}{c}\text { Medium Density Mixed } \\
\text { (minutes) }\end{array}$ \\
\hline vial 10 (inside original packaging) & did not exceed & 35 \\
vial 20 (in tray, lower shelf) & did not exceed & $53^{\star}$ \\
vial 11 (inside original packaging) & did not exceed & $57^{*}$ \\
vial 13 (syringe in box - top) & did not exceed & did not exceed \\
vial 12 (inside cardboard box) & did not exceed & did not exceed \\
vial 6 (inside box, floor level) & did not exceed & did not exceed \\
\hline
\end{tabular}

*Although $8{ }^{\circ} \mathrm{C}$ limit crossed, maximum temperature was less than $8.1{ }^{\circ} \mathrm{C}$

During high density load trial, all of the vials remained within the specified temperature range despite the door being left ajar for $60 \mathrm{~min}$. In the medium density trial, nearly all the vials remained within range, except for vial 10 which exceeded $8{ }^{\circ} \mathrm{C}$ after 35 minutes. 
However, even this vial only reached a maximum temperature of $8.7^{\circ} \mathrm{C}$ at the end of the trial. Vials 20 and 11 both exceeded the upper limit towards the end of the hour long test, but by less than a tenth of a degree. In either case, these small excursions are not likely to impact the potency of the vaccines. The differences between the high density and medium density trials can probably be attributed to the additional thermal mass in the high density trial contributing to resist temperature change, much in the same way that the water bottle temperature ballast worked in our tests of the other refrigerators.

Overall, the pharmaceutical refrigerator model performed very well in both the dooropening and door left ajar trials. The refrigerator exhibited excellent temperature control, compensating for the less than ideal operating conditions by adjusting the cooling output to maintain proper vaccine storage temperatures.

\subsubsection{Effect of Increasing Room Temperature}

To determine whether room temperature fluctuations impact pharmaceutical refrigerator's ability to maintain its set point temperature, we performed three trials in which the room temperature was varied between $24{ }^{\circ} \mathrm{C}$ and $29^{\circ} \mathrm{C}$. We can analyze the results in the same manner as in the dual-zone refrigerator trials, by comparing the room temperature probe readings from logger LD to the refrigerator probe readings from LD, LE1 and LE2. This analysis is summarized in table 10 below.

Table 10. Refrigerator probe temperature change relative to room temperature change $\left({ }^{\circ} \mathrm{C}\right)$

\begin{tabular}{|c|c|c|c|}
\hline Logger Name & High Density Mixed I & High Density Mixed II & Medium Density Mixed \\
\hline LD & -0.02 & -0.02 & -0.02 \\
LE1 & -0.01 & -0.02 & 0.02 \\
LE2 & 0.00 & 0.01 & -0.01 \\
\hline
\end{tabular}

Table 10 shows the temperature change recorded by each of the three refrigerator data logger probes scaled to the change in ambient temperature recorded by external probe. So, for example, during the High Density Mixed with Bottles I trial, logger LE1 recorded a temperature decrease of $0.01{ }^{\circ} \mathrm{C}$ for every one degree increase in room temperature.

As with trials using the dual-zone refrigerator, the pharmaceutical refrigerator model does not show correlated interior vs. exterior temperature behavior. Again, the inherent complexity of this relationship prevents us from making definitive statements based on a brief study of the issue. However, we can note that the degree by which the internal refrigerator temperature is affected by external room temperature changes is even smaller in the pharmaceutical refrigerator than in the dual-zone model. All three data logger probes recorded a temperature change of less than $\pm 0.02{ }^{\circ} \mathrm{C}$ per degree rise in room temperature. This effect is so small that it may be a product of extraneous factors rather the changing room temperature. If we assume these changes are in fact due to room temperature variation and that the relationship is linear, we would need to vary environmental conditions by $50{ }^{\circ} \mathrm{C}$ just to see a one degree change in internal refrigerator temperature. 
The pharmaceutical refrigerator's performance in these trials indicates that it should be able to withstand even larger ambient temperature changes than the dual-zone refrigerator while maintaining proper vaccine storage conditions in its interior. However, it must be emphasized that because our study only monitored the effects of a $5{ }^{\circ} \mathrm{C}$ room temperature change, we may not have captured additional thermal effects that could occur during much larger room temperature changes. The use of a continuous temperature monitoring system will allow workers to immediately identify any problem temperature effects resulting from different environmental conditions.

\subsubsection{Effect of a Power Outage}

To observe what occurs following a power outage, we performed two trials in which the closed refrigerator was unplugged and monitored overnight. In both trials, all of the thermometers recorded a fairly steep increase in temperature that slowed down slightly over time. All of the thermometers, including those attached to vaccine vials, exceeded the allowed maximum temperature within 1:46 h:min for the medium density trial, and within 2:19 h:min during the high density trial. Figure 23 below shows the temperature readings of all of the thermometers during the high density load power off trial. The sudden drop in temperature following the peak corresponds to the point at which the refrigerator was plugged back in.

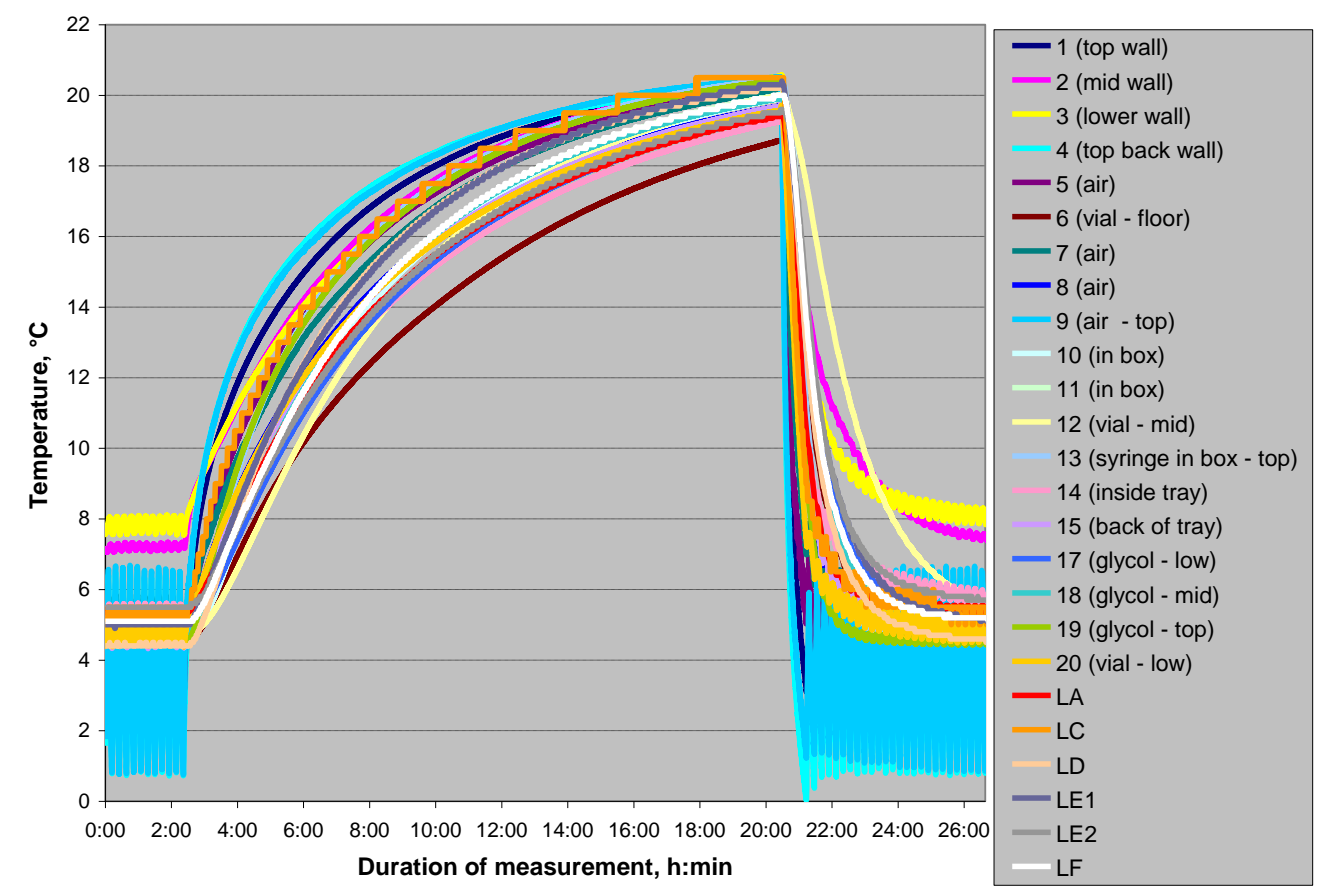

Fig. 23. Thermometer response during a power-outage event. High density mixed load trial.

The next graph (Fig. 24) shows the data collected starting from the time that the refrigerator was unplugged. The dashed red line indicates the $8{ }^{\circ} \mathrm{C}$ upper limit, and the blue dashed line shows the $2{ }^{\circ} \mathrm{C}$ minimum. 


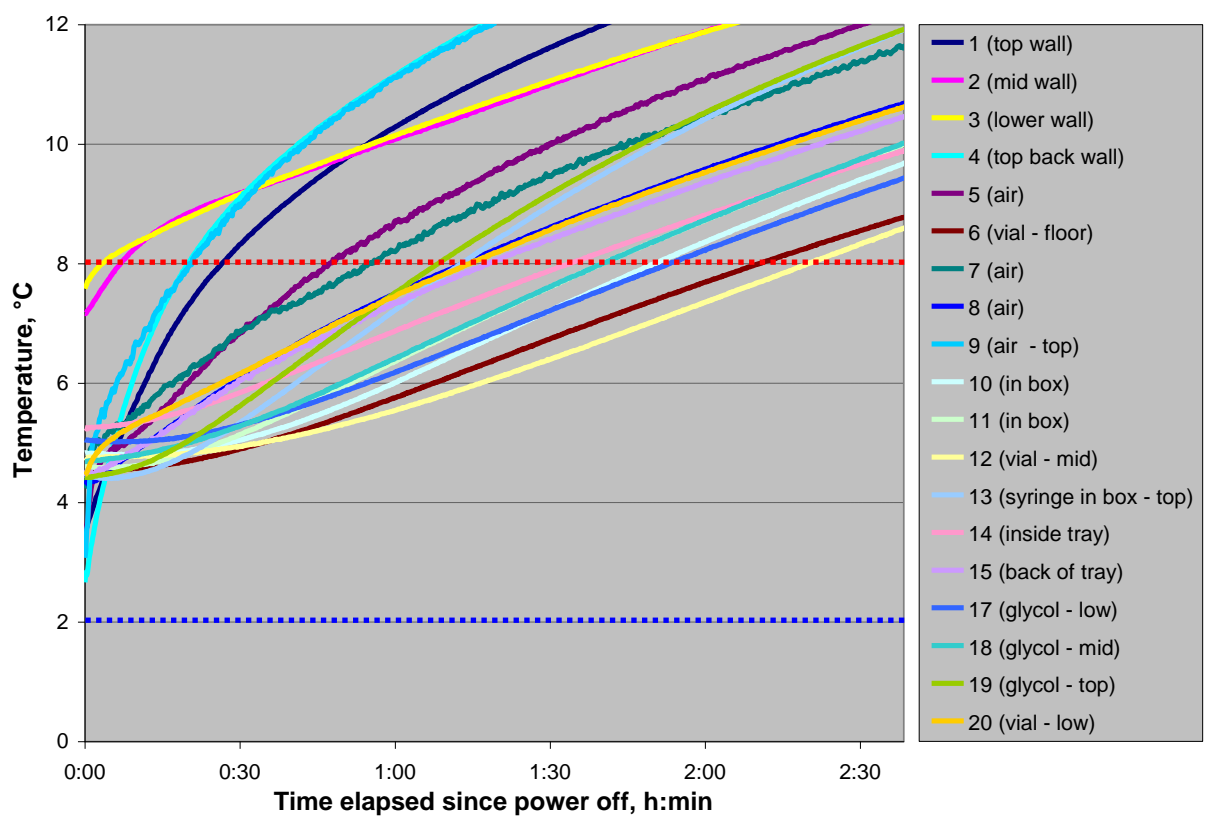

Fig. 24. Expanded view of thermometer response during a power-outage event. High density mixed load trial.

To determine the required response time to preserve vaccine viability during a power outage situation, we examined the amount of time elapsed from the point that the refrigerator was unplugged until the thermometers attached to vials exceeded $8{ }^{\circ} \mathrm{C}$. This information is reported in Table 11 below.

Table 11. Time after power-off until vial temperature exceeded $8{ }^{\circ} \mathrm{C}$.

\begin{tabular}{|l|c|c|}
\hline \multicolumn{1}{|c|}{ Name and Location } & $\begin{array}{c}\text { High Density Mixed } \\
\text { (h:min) }\end{array}$ & $\begin{array}{c}\text { Medium Density Mixed } \\
\text { (h:min) }\end{array}$ \\
\hline vial 20 (in tray, lower shelf) & $1: 14$ & $0: 45$ \\
vial 13 (syringe in box - top) & $1: 12$ & $0: 50$ \\
vial 11 (inside original packaging) & $1: 40$ & $1: 01$ \\
vial 10 (inside original packaging) & $1: 50$ & $0: 58$ \\
vial 6 (inside box, floor level) & $2: 10$ & $1: 15$ \\
vial 12 (inside cardboard box) & $2: 19$ & $1: 46$ \\
\hline
\end{tabular}

From this data, we can immediately see that vials kept in the powered-off pharmaceutical refrigerator were subjected to out-of-range temperatures much more quickly than in trials of the other refrigerator models. All of the vials exceeded $8{ }^{\circ} \mathrm{C}$ in less than $2.5 \mathrm{~h}$, and in some cases, less than $1 \mathrm{~h}$, depending on vial storage method. This does not leave a very large window of time for clinic workers to recognize the occurrence of a power outage and implement a backup storage method before vaccines are impacted by the prolonged exposure to temperatures above the upper limit. While the pharmaceutical refrigerator we studied appeared to perform exceptionally well as a vaccine storage unit across the range of tested criteria, including different loading configurations, variable room temperature conditions, and operation with the refrigerator door left open, the results of the power outage tests identify a significant limitation of this refrigerator model. The doors of the 
pharmaceutical refrigerator model used in our tests were the sliding glass variety. Some users may prefer glass doors since they are able to easily inventory refrigerator contents without exposing vaccine vials to room temperature air. However, a major known drawback to the glass doors is that they lack the insulation provided by a standard opaque door. This clearly becomes an issue in the event of a power outage. Once the cooling unit inside the refrigerator cuts out, warm ambient air is slowly conducted into the refrigerator interior, eventually warming the contents until the temperature inside and outside the unit is in equilibrium. If the refrigerator door consists of only thin glass panels and rubber gasket, heat is conducted to the interior much more quickly than if it had to travel through a heavily-insulated, solid refrigerator door.

Even so, the limitations of glass refrigerator doors during a power outage event should not discount pharmaceutical refrigerator models as viable vaccine storage units. First of all, many pharmaceutical grade models feature solid, insulated-type refrigerator doors. These units are likely to have robust temperature stability, similar to our tested unit, with the added benefit of increased resistance to heating during outage events. Regardless of the glass doors issue, it is perhaps most important for each individual provider office to evaluate their own circumstances and determine which of many possible risks are the most critical to their situation. For example, the average pharmaceutical grade refrigerator user may have different office and environmental conditions than a provider office with a standard household refrigerator. Hospitals and higher budget clinics are more likely to have access to the pricier pharmaceutical refrigerators, while smaller budget operations are likely to choose less expensive household models. The differences between these two settings are likely to extend beyond just their vaccine storage refrigerator of choice. The high-budget, modern hospital may have an excellent backup generator system in place to keep equipment running through power outages. In addition, the hospital has workers on duty 24-7, so risk the of a brief power outage going undetected is low. By contrast, a private practice operating out of a leased building might not have as much control over their power supply. This type of practice is also less likely to have workers on-site at all times, so an overnight power outage poses a greater risk.

While the glass door pharmaceutical refrigerator model did not resist thermal excursions during our power outage trials as well as some of the household models, the needs of the end user should be carefully evaluated before making blanket statements about which refrigerator is better based on a single criteria. For some settings, the single greatest threat to drug potency may be the direct result of high vaccine demand, such that the storage refrigerator door is constantly opened to retrieve vaccines. In this case, choosing the pharmaceutical refrigerator for its ability to maintain stable temperatures despite these conditions makes sense, even if it is at the expense of a more insulating door designed for resistance to power outages.

Consistent with the results from other trials, we observed that vials contained in boxes or other packaging materials resisted thermal excursions for a longer time than unpackaged vials. This occurs because additional time is required for heat to transfer through the exterior packaging material before the vial temperature is affected. 


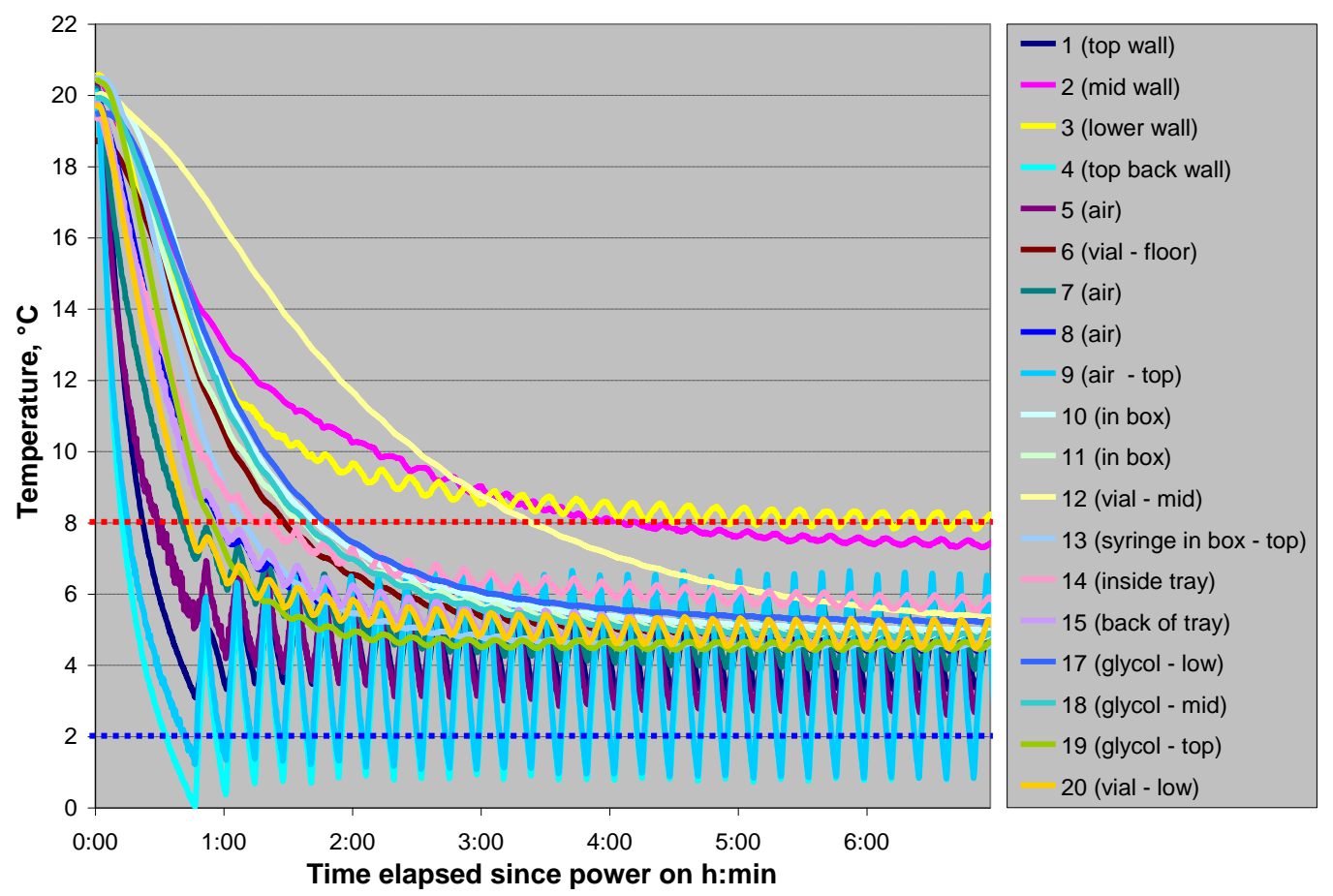

Fig. 25. Recovery after restoration of power for a pharmaceutical refrigerator.

In both trials, the pharmaceutical refrigerator reached its pre-outage equilibrium temperature state approximately $7 \mathrm{~h}$ after being powered.

\subsubsection{Defrost Cycle in the Pharmaceutical Refrigerator}

The pharmaceutical refrigerator model we tested features a defrost heater which is activated each time the refrigerator compressor is cycled off. According to the manufacturer, the defrost cycle will not have any discernible effect on the chamber temperature. Throughout the range of testing, we did not detect any interior temperature effects associated with the defrost cycle, nor did we observe accumulated frost. In the event of frost accumulation on the evaporator, which may occur under high-humidity conditions, a forced defrost cycle may be initiated.

\subsubsection{General Stability of Vial Temperature and Suitability of Pharmaceutical Refrigerator Model for Maintenance of Vaccine Storage Conditions}

Our results indicate that the pharmaceutical refrigerator model is suitable for vaccine storage. Vaccine vial temperatures remained within the specified $2{ }^{\circ} \mathrm{C}$ to $8{ }^{\circ} \mathrm{C}$ range throughout all of the packing variation trials, as well as during the 15 second door opening trials. Furthermore, average vial temperatures varied by less than $1.5{ }^{\circ} \mathrm{C}$ throughout this entire range of testing. The pharmaceutical refrigerator exhibits excellent long-term temperature stability, even under varying conditions. 


\subsubsection{Best and Worst Locations for Vial Storage in the Pharmaceutical Refrigerator}

Throughout the course of the closed-door pharmaceutical refrigerator trials, vaccine vials remained within the desired temperature range regardless of their location in the refrigerator. This refrigerator model is purpose-built for vaccine storage. As a result, it features enhanced temperature set point control and more a sophisticated ventilation system than is included in most household refrigerator models. Because of this, vials stored in different areas of the pharmaceutical refrigerator were kept within a narrow temperature range of $1.5^{\circ} \mathrm{C}$ or less.

However, based on the results of the door opening and power-off trials, it appears that some storage methods and locations within the refrigerator may provide slightly better thermal stability than others. Just as we saw in trials with other refrigerator types, vials stored inside thermally isolated containers experienced less temperature disruption during normal, periodic door-opening operation than unpackaged vials kept in trays. In the pharmaceutical refrigerator trials, this translated to an unpackaged vial experiencing a temperature spike of $0.4{ }^{\circ} \mathrm{C}$ each time the refrigerator door was opened, whereas the vials in boxes showed no discernible temperature spike. Similarly, during the power outage trials, the first vial to exceed $8{ }^{\circ} \mathrm{C}$ was kept unpackaged and in an open tray, while the last vial to exceed the limit was stored inside a corrugated cardboard box.

Overall, the pharmaceutical refrigerator proved to be less resistant to thermal excursions during a power outage than some of the household refrigerator models tested. This can mostly likely be attributed to the pharmaceutical model's glass sliding doors. The glass doors transfer heat to the interior of the refrigerator more quickly than more insulated doors. As a result, vaccines stored in a glass-door pharmaceutical refrigerator may be more susceptible to damage during a power outage event than those stored in a household refrigerator with well insulated doors. On the other hand, we found that the pharmaceutical model is able to consistently maintain vaccine storage temperatures with the door left open for prolonged periods of time. Because of this, when using a glass-door pharmaceutical refrigerator, it might be advantageous to thoroughly package vaccines in boxes or insulating material. If workers spend extra time with the refrigerator door open while getting vials in and out of the packaging, it is not likely to impact vaccines negatively because of the refrigerator's robust temperature control. At the same time, the extra packaging will provide an additional layer of protection against heat transfer from ambient air in the event of a power outage.

Throughout testing of the pharmaceutical refrigerator, we intentionally kept one vial on the floor level of the refrigerator, below the bottom wire shelf. The manufacturer instructions specifically state not to store items on this level. However, the vial placed on the floor remained within the specified temperature range across all trials, and its temperature behavior mapped that of vials stored in other areas for most of the trials. This vial did tend to record colder temperatures than the rest of the vials, but the difference was generally less than $1{ }^{\circ} \mathrm{C}$. So while we do not explicitly recommend vaccine storage in this location, if workers inadvertently placed vials on the floor level, or were forced to, for lack of other space, the vaccines probably would not be negatively affected. 
The only thermocouples that showed significant deviations from the tightly-controlled, $4{ }^{\circ} \mathrm{C}$ to $5{ }^{\circ} \mathrm{C}$ temperature distribution recorded by the majority of the thermometers were those attached to the refrigerator walls. In particular, two thermocouples on the upper refrigerator walls consistently recorded temperatures above $8{ }^{\circ} \mathrm{C}$ across all of the trials. Our testing did not include the temperature behavior of vials placed in direct contact with the refrigerator walls, but it is possible that such contact could cause the vials to experience thermal excursions as heat if conducted from the walls. By keeping stored vaccines several inches away from the walls, workers can avoid any such nonstandard temperature effects.

\subsubsection{Best and Worst Methods of Monitoring Vial Temperature}

As with the dual-zone refrigerator results, we can determine the suitability of various methods for monitoring vial temperature in the pharmaceutical refrigerator by comparing the readings of vial-attached thermocouples to readings of other thermometers placed throughout the refrigerator. 

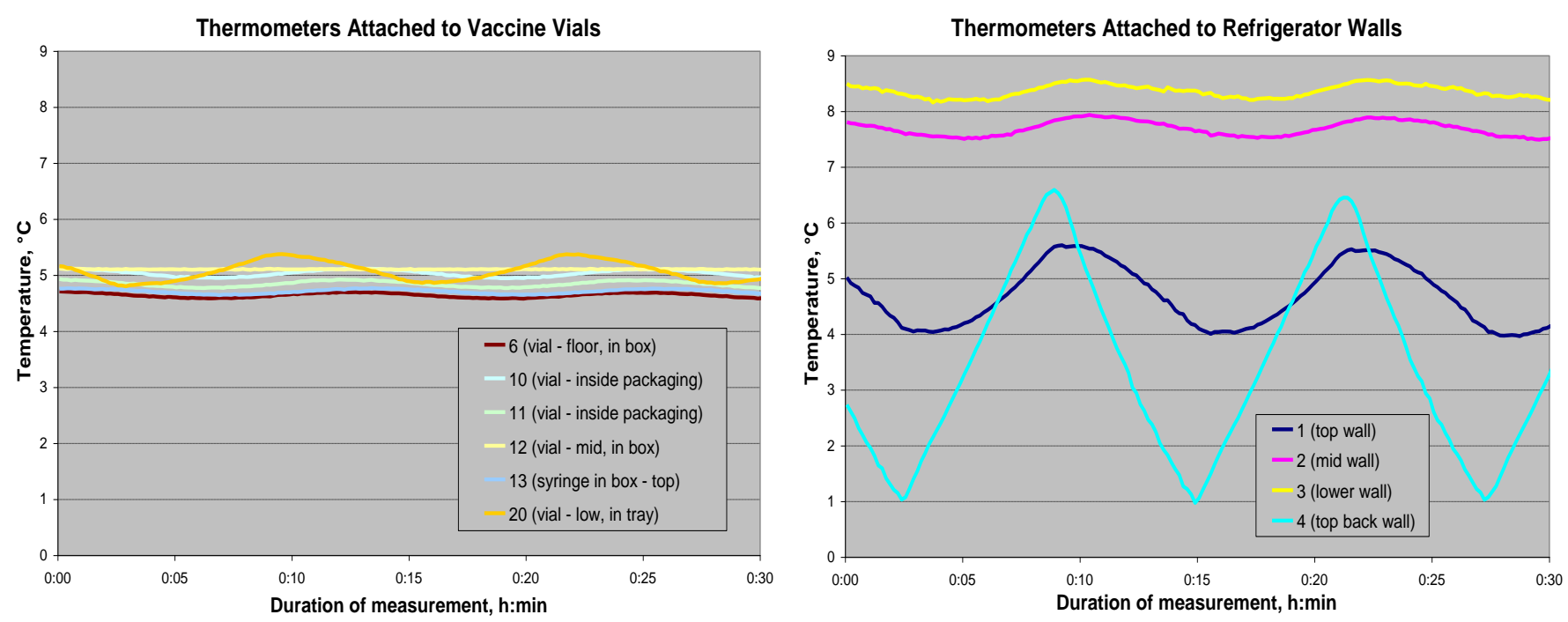

Thermometers Inside Glycol-filled Bottles
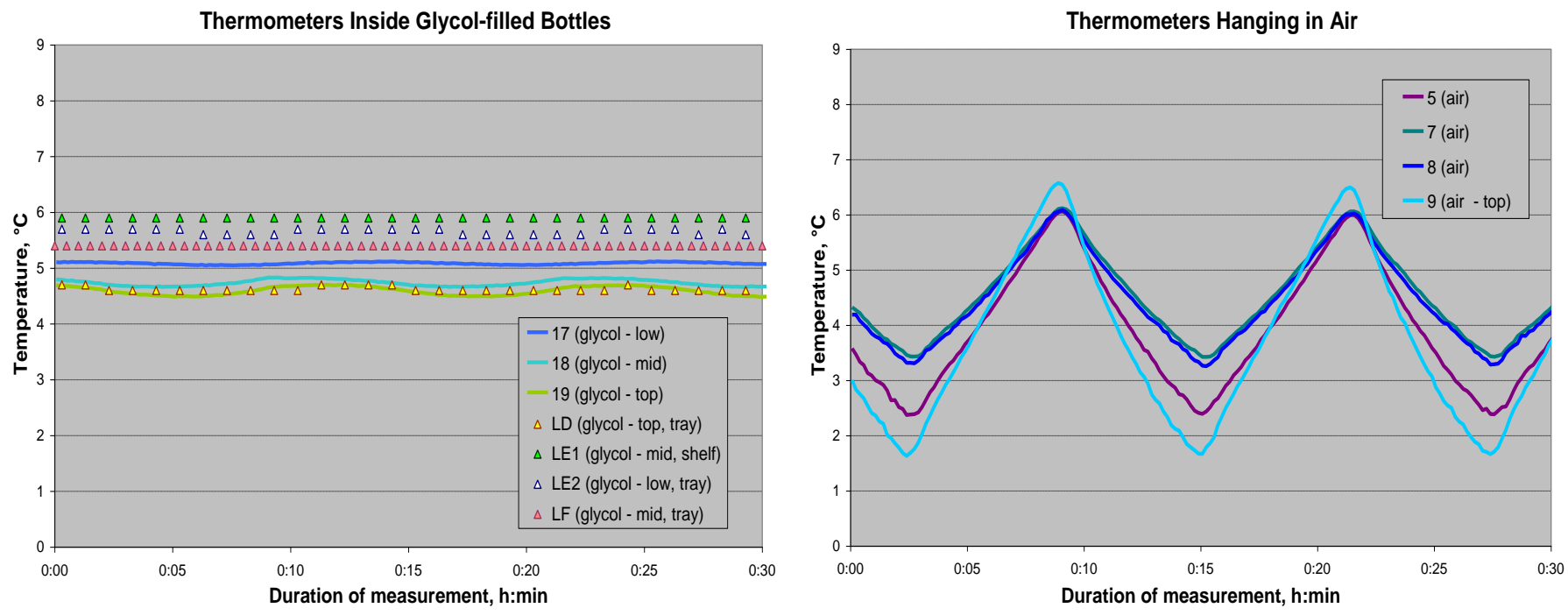

Thermometers in Trays or on Shelves
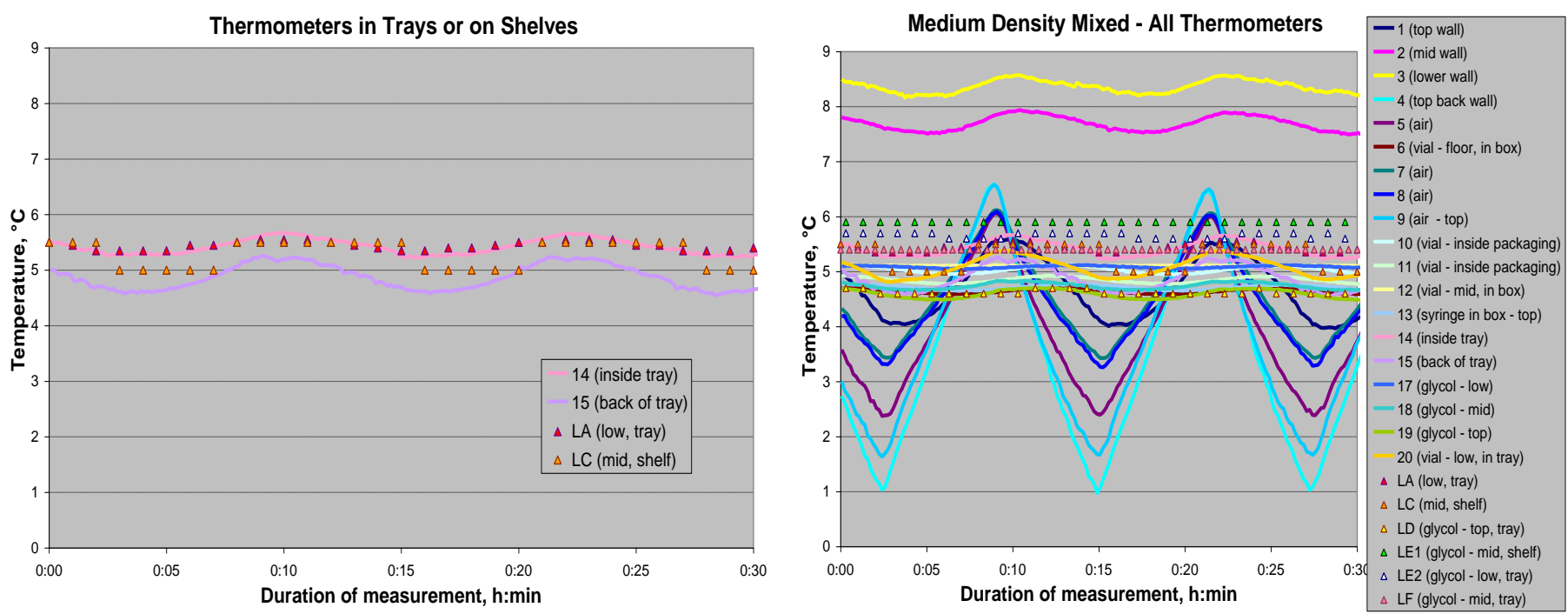

Fig. 26. Response of thermometers with different mounting methods. 
The pharmaceutical refrigerator maintained vaccine vials within a very narrow temperature window throughout the course of our study. During normal, closed-door operation, as shown in figure 26 above, vial temperature variation due to both refrigerator compressor cycling and varying storage location was less than $1{ }^{\circ} \mathrm{C}$. Even during door opening trials, vial temperatures did not fluctuate by more than $1{ }^{\circ} \mathrm{C}$, regardless of how the vials were packaged or where they were stored. Examining the above graphs, we can essentially divide the remaining thermocouples and data loggers into two groups: thermometers that closely followed the thermal behavior of the vial-attached thermocouples, and thermometers that recorded very different temperature behavior than the monitored vials.

\section{Good Locations for Temperature Sensors}

Thermometers that closely matched vial temperatures were stored in trays or on refrigerator shelves (TC \#s 14, 15, 17, 18, 19; data loggers LA, LC, LD, LE1, LE2, LF). Like the monitored vaccine vials, the temperature distribution of these thermometers stayed centered about $4.5{ }^{\circ} \mathrm{C}$ to $5.5{ }^{\circ} \mathrm{C}$ throughout the range of testing. Whether or not the thermometer had a probe inside a glycol-filled bottle did not impact its ability to predict vial temperature to within a desirable range, which we define as $\pm 1{ }^{\circ} \mathrm{C}$. The thermometers with probes in glycol-filled bottles showed nearly constant temperature profiles over time, similar to the data collected by the vials stored inside thermally-isolating boxes or packaging. By contrast, the standalone data loggers with built-in probes as well as the thermocouples attached directly to trays both showed small temperature fluctuations comparable to the variations recorded by the unpackaged vial stored in a tray. Because the pharmaceutical refrigerator appears to maintain its contents within a very small, consistent temperature window $\left( \pm 1^{\circ} \mathrm{C}\right)$, placing temperature monitors anywhere inside the refrigerator where vaccines are normally stored produces readings that are representative of actual vial temperatures, regardless of whether the monitors are kept on a shelf or in a tray, and with or without a probe in a glycol-filled bottle.

\section{Unacceptable Locations for Temperature Sensors}

Thermometers attached to refrigerator walls or hanging in the air (TC \#s 1, 2, 3, 4, 5, 7, 8, 9 ) were poor indicators of actual vaccine vial temperature. While the pharmaceutical refrigerator we tested excelled at keeping its contents within a small, stable temperature band, the refrigerator walls and air experienced large temperature fluctuations (amplitudes of $1.5^{\circ} \mathrm{C}$ to $6^{\circ} \mathrm{C}$ or greater) corresponding to the refrigerator cooling cycle. In addition, two thermocouples taped to the interior left wall of the unit, $(2,3)$, consistently recorded mean temperatures between $7^{\circ}$ and $9{ }^{\circ} \mathrm{C}$, while vial temperatures remained around $5^{\circ} \mathrm{C}$. Relying on a thermometer attached anywhere to the pharmaceutical refrigerator's interior walls or hanging in the airspace is clearly a poor choice for vaccine temperature monitoring. Many of these thermometers repeatedly experienced thermal excursions outside the required $2{ }^{\circ} \mathrm{C}$ to $8{ }^{\circ} \mathrm{C}$ storage temperature range, while vaccine temperatures remained nearly constant between $4.5^{\circ} \mathrm{C}$ and $5.5^{\circ} \mathrm{C}$. Based on these results, thermometers used for vaccine temperature monitoring should not be kept in contact with refrigerator walls or hung in the air. This practice can result in 
false out-of-range temperature alarms, leading to unnecessary disposal of still-viable vaccine or adjustment to an improper set point temperature.

\section{DATA LOGGER EVALUATION}

Throughout the course of trials performed on both refrigerators, we tested the performance of several electronic temperature loggers along with the thermocouples. The data loggers tested, labeled LA, LC, LD, LE1, LE2, and LF are described in Table 3. We wished to evaluate the data loggers' effectiveness at monitoring vial temperature.

At present, many VFC clinics use digital display, analog, or liquid in glass thermometers to monitor the temperature of their vaccine storage refrigerators. They are only required to check and record the temperature readings of these thermometers twice each working day. Based on the results of our study, a number of factors, including the frequency of refrigerator door opening, refrigerator defrost cycles, room temperature increases, and power outages, could cause an employee to obtain a false representation of the actual refrigerator and/or vial temperature based on this methodology. For example, an overnight power or AC outage could cause the refrigerator and corresponding vaccine temperatures to rise outside of the viable range. If the outage is corrected before the next morning, the refrigerator may return to its cooled equilibrium state by the time employees come to work and check their temperature monitoring devices. In this case, they would fail to detect that the vaccine vials exceeded the allowed temperature range overnight, rendering them potentially unusable. This type of situation could pose a serious danger to clinic patients, since they could be unknowingly administered an ineffective vaccine.

In the same light, other conditions could cause employees to record a "false positive" temperature, and discard vaccines unnecessarily. Depending on a thermometer's accuracy, its placement inside the refrigerator, and the time that it is checked, its reading may or may not correspond to actual vaccine vial temperature. Some thermometers are more sensitive to temperature fluctuations than the fluid-filled vaccine vials, which have a larger thermal mass. If an employee happened to read one of these thermometers during the refrigerator's standard defrost cycle, which produces a brief air-temperature spike, he or she might determine that the vaccines had exceeded the allowed temperature limit. In our experiments, we found that the actual vial temperatures were not greatly affected by the defrost cycle, but that some of the other thermometers indicated significant temperature increases. Because of these issues, we believe it is necessary for vaccine storage refrigerators to have in place continuous data loggers to obtain truly representative, reliable readings of actual vaccine temperatures.

In addition to providing continuous temperature data, the widespread use of data loggers in VFC clinics would reduce the overall workload involved in refrigerator temperature monitoring. At present, VFC workers must manually record refrigerator temperature two times a day. The VFC coordinator is then responsible for reviewing hand-written temperature logs to determine if any lapses occurred. Electronic data loggers connect to a computer via USB and can quickly download data through easy-to-use software 
interfaces. Logger data for multiple days are automatically summarized into spreadsheets and graphs in a matter of minutes, relieving the VFC coordinator of all data entry and analysis work. As a result, we believe that the use of data loggers for vaccine temperature monitoring would not only improve the reliability this process, but also make it significantly more efficient.

All of the data loggers we tested produced temperature data close to that of the vialattached thermocouples. Throughout our study, we saw that refrigerators do not maintain a completely uniform temperature profile throughout their interiors, but that the temperature varies according to location. As such, it would be impossible for a single data logger to capture the temperatures of all of the vials stored throughout the refrigerator at any given time. Instead, a logger used to monitor stored vaccines should produce data that is representative of vial temperature behavior, modeling temperature trends over time and within $0.5^{\circ} \mathrm{C}$ to $1{ }^{\circ} \mathrm{C}$ of the range of vial temperatures.

During our study of the pharmaceutical and dual-zone refrigerator models, we saw that certain data loggers modeled vaccine vial temperature more closely than others. However, all of the data loggers consistently recorded temperatures to within $1{ }^{\circ} \mathrm{C}$ of nearby vaccine vial temperatures over the entire range of tests.

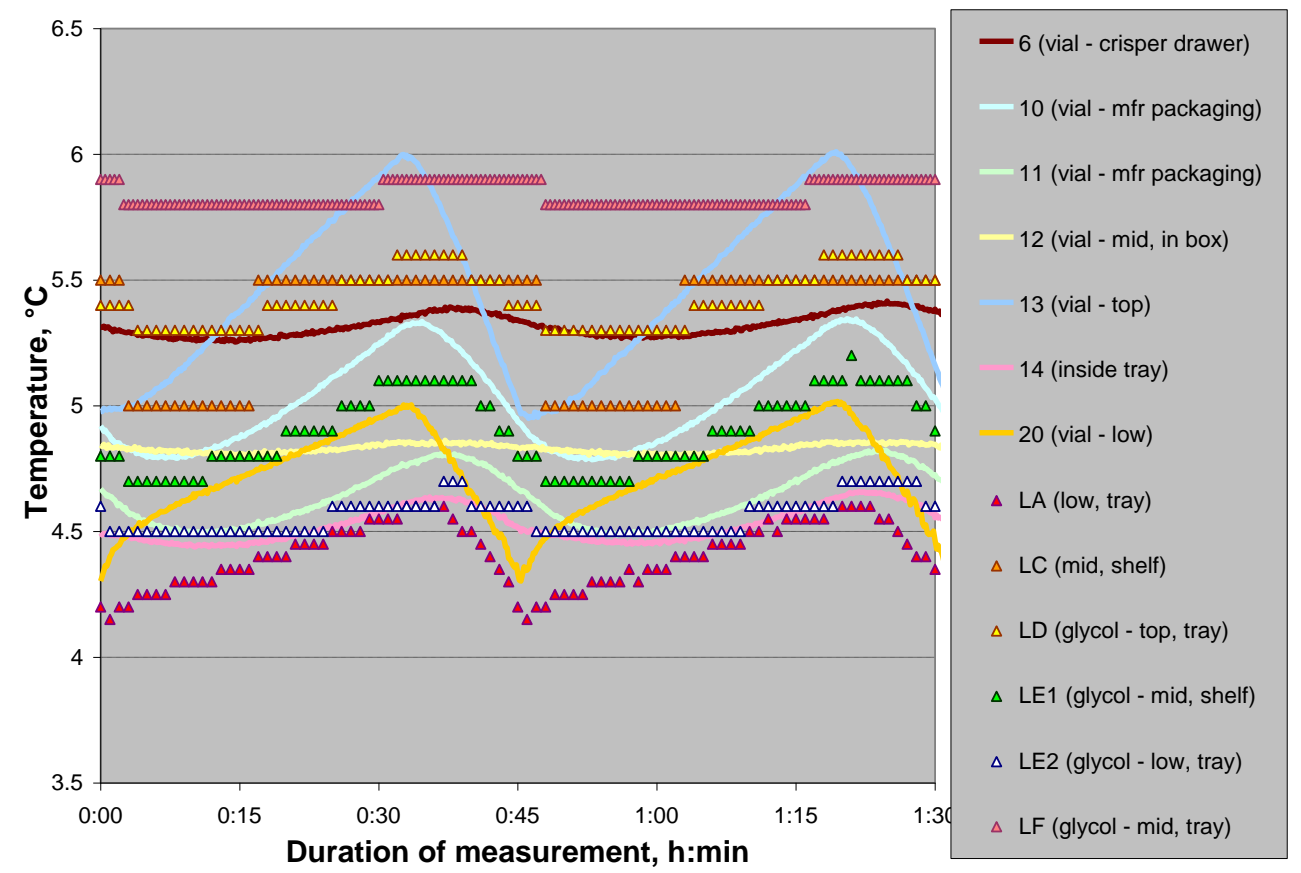

Fig. 27. Sample of temperature data from a closed door dual-zone refrigerator trial, comparing vial temperatures and data logger measurements.

Figure 27 above shows a selection of data from a dual-zone refrigerator trial. Temperatures recorded by thermocouples attached to vaccine vials are shown as solid color lines, and electronic data logger readings are shown as triangular markers. Note that vertical span of data is less than $2{ }^{\circ} \mathrm{C}$. From this, we can see that all of the loggers reported values closely matching actual vial temperatures. The differences between 
individual vaccine vial and data logger temperatures are relatively small, and are most likely caused by location-specific temperature gradients or varying storage method inside the refrigerator. Our primary concern is maintaining the refrigerator and its contents between $2{ }^{\circ} \mathrm{C}$ and $8{ }^{\circ} \mathrm{C}$, so these temperature differences $\left({ }^{\circ} \mathrm{C}\right.$ or less) are not likely to be a major issue.

In order to effectively monitor vaccine stores, electronic data loggers must successfully capture any thermal excursions that occur under variable conditions such as repeatedly opening the refrigerator door, initiation of an automated defrost cycle, or a power outage event. Overall, we saw that the tested loggers replicated the temperature responses of the vial-attached thermocouples during these events, but to varying degrees of sensitivity. Logger sensitivity is affected by both its placement inside the refrigerator as well as the inherent design properties of the device. The various data logger models we tested can be divided into one of two main design types. The first type is a standalone unit with a builtin temperature sensor (data loggers LA, LC). In this case, the entire unit is placed inside the refrigerator for the duration of the measurement period, and then removed in order to connect and download the data to a computer. The second type of data logger we tested featured a main logger unit with an external temperature probe attached via cable (data loggers LD, LE1, LE2, LF). With this type of logger, we were able to keep the main unit outside of the refrigerator while the temperature probe was positioned inside the refrigerator. In order to download recorded measurements, the main unit could be detached from the probe and connected to a computer without opening the refrigerator door and removing the temperature sensor to ambient conditions. Throughout our study, the external probes of each of these devices were kept inside glycol-filled bottles. This provided additional thermal mass, similar to that of the liquid vaccine inside glass vials. As expected, the standalone data logger units responded to temperature changes under varying conditions more quickly than the external probes kept in glycol. This is because it takes longer for the entire mass of the glycol to change temperature than it does for the air surrounding a standalone data logger unit.

In terms of sensitivity to temperature change, we did not find one logger type to be especially advantageous over the other. For example, during the door opening trials, neither logger type registered much of a temperature spike each time the door was opened, most likely because the net internal temperature change of the refrigerator was both small and brief. During these trials, we found that the vaccine vials kept in boxes or their original packaging also did not record temperature spikes in response to the door opening. Only unpackaged vials kept directly on a shelf or in an open tray experienced noticeable temperature fluctuations in response to opening and closing the refrigerator door (e.g. TC \#20).

In addition, the performance of loggers of each type varied between individual units. Of the loggers with external probes kept in glycol, logger LD tended to record temperature fluctuations consistent with the behavior recorded by packaged vaccine vials. By contrast, logger LF consistently measured an almost flat line temperature profile. Whether these types of variations are due to differences in device resolution, probe material, device placement within the refrigerator, or some other combination of factors 
is unclear. Comparing the two standalone data logger units (LA, LC), we saw that LC was consistently more sensitive to temperature fluctuations than LA. In this case, the discrepancy can most likely be attributed to the materials that the loggers are constructed from. Logger LA features a heavy-duty metal sheath capable of enduring high temperatures and total submersion, while LC is mainly composed of plastic. As a result, the larger thermal mass of LA means that the logger takes a longer time to experience temperature changes. In this application, we found that the heavy-duty construction of LA may be a disadvantage. Each time we removed logger LA from the refrigerator to download data, we found that it required as much as 3 or $4 \mathrm{~h}$ cool down once it was placed back inside the refrigerator. During this time period, the logger is not very useful as a vial temperature monitoring device. By contrast, data logger LC, with its lighter plastic exterior, required approximately one hour of equilibration time once it was taken from room temperature and placed inside the refrigerator.

During the simulated power outage trials, all of the data loggers recorded rates of temperature increase similar to that experienced by the vaccine vials. The length of time before the data loggers exceeded $8{ }^{\circ} \mathrm{C}$ generally fell within $15 \mathrm{~min}$ of the time that nearby vials also exceeded $8{ }^{\circ} \mathrm{C}$. In general, if the data loggers are stored in the same fashion as the vaccine vials, whether in trays or inside cardboard boxes, and in the same part of the refrigerator, they will most likely give readings within their resolution (Table 3) of the actual vial temperatures. However, during a power outage, unless the problem can be remedied within an hour or two, the vaccines are likely to become unusable. In this case, having a continuous data logging system in place is critical to maintaining confidence in vaccine supply efficacy. Without a continuous temperature monitoring system, workers might have no way to tell if a temporary outage occurred outside of working hours, possibly damaging vaccines. All of the data loggers we tested clearly demonstrated the temperature effects of the power outage on a similar time scale to the monitored vaccine vials. As such, any of the data loggers we tested could be used to determine whether vaccine supplies have been subjected to inappropriate storage temperatures.

Although all of the data loggers we tested performed in a manner acceptable for vaccine temperature monitoring and management, we found that some loggers featured design characteristics that made them more efficient or user friendly than others. For example, the loggers featuring external temperature probes (LD, LE1, LE2, LF) eliminate the need to wait for thermal equilibrium after replacing a room temperature logger back into a refrigerator, since the external probes could be kept in a glycol filled bottle inside the refrigerator, while the main device unit was kept outside of the refrigerator. To download data, we simply detached the main unit from the cable, leaving the probe inside the refrigerator. The main unit was then connected to a USB data collection cradle, which allowed us to transfer the data to a computer. The main unit of each of these loggers also featured a readout display, enabling users to visually check current refrigerator conditions without having to open the door or download data. In addition to its external probe kept inside the refrigerator, logger LD also featured an internal sensor inside its main readout unit. If the main unit is kept outside the refrigerator, this device can simultaneously monitor room temperature and refrigerator temperature. Logger LF was enabled for wireless data transmission and download, a feature that could be especially useful in 
circumstances like an overnight or weekend power outage. Users can configure the device so that real-time refrigerator conditions can be checked from any computer with an internet connection. With a system like this in place, VFC providers could detect and deal with any potential refrigeration temperature problems immediately, rather than discovering ruined vaccines after the fact.

Overall, the tested data loggers' performance seems to be sufficient for monitoring vaccine temperatures. The most important factor for temperature monitoring accuracy is the placement of the device inside the refrigerator. Regardless of the type of data logger chosen, it should be stored in the same manner as the vaccines in order obtain the most accurate readings. Simply placing a data logger directly on a refrigerator shelf is not likely to give the most accurate representation of vial temperatures. If the vaccine vials are stored inside trays or boxes, the data loggers should be stored in the same way.

In the next phase of our vaccine storage and handling study, we will perform a more detailed investigation of electronic thermometers used for cold-chain measurements. In this investigation, we will test a minimum of five data logger thermometers for out-ofbox performance, evaluating the manufacturer specified accuracy over the temperature range of $0{ }^{\circ} \mathrm{C}$ to $10^{\circ} \mathrm{C}$. In addition, we will quantify the stability of each logger over a six month period and determine the proper use within a vaccine storage system so that temperature measurements reflect vaccine vial temperatures. 


\section{SUMMARY}

Table 9 below summarizes the results obtained in this study.

Table 9. Comparison of refrigerator performance in response to tested criteria.

\begin{tabular}{|c|c|c|}
\hline \multicolumn{3}{|c|}{$\begin{array}{l}\text { COMPARISON OF REFRIGERATOR PERFORMANCE } \\
\text { IN RESPONSE TO TESTED CRITERIA }\end{array}$} \\
\hline Criteria & Dual-Zone Refrigerator & $\begin{array}{l}\text { Pharmaceutical } \\
\text { Refrigerator }\end{array}$ \\
\hline Loading density & $\begin{array}{l}\text { - Slight impact on performance } \\
\text { - High-density loading patterns } \\
\text { increased location-specific } \\
\text { temperature variation }\end{array}$ & - No impact on performance \\
\hline $\begin{array}{l}\text { Packing style } \\
\text { (Trays, Boxes or Mixed) }\end{array}$ & $\begin{array}{l}\text { - No noticeable impact on } \\
\text { performance }\end{array}$ & $\begin{array}{l}\text { - No noticeable impact on } \\
\text { performance }\end{array}$ \\
\hline $\begin{array}{l}\text { Opening/ closing } \\
\text { refrigerator door }\end{array}$ & $\begin{array}{l}\text { - Vial temperatures showed small } \\
\text { increases in response to door } \\
\text { opening, but remained in } 2{ }^{\circ} \mathrm{C} \text { to } \\
8{ }^{\circ} \mathrm{C} \text { range throughout trial } \\
\text { - Thermometers in air / attached } \\
\text { to walls exceeded } 8{ }^{\circ} \mathrm{C} \\
\text { - Thermometers in close } \\
\text { proximity to cooling fan } \\
\text { recorded temperature decreases } \\
\text { below } 2{ }^{\circ} \mathrm{C} \text {, as the refrigerator } \\
\text { pumped out additional cold air } \\
\text { to compensate for the door } \\
\text { opening } \\
\text { - Keeping water bottles in door } \\
\text { did not have a major impact on } \\
\text { temperature stability }\end{array}$ & $\begin{array}{l}\text { - Excellent temperature } \\
\text { stability preserved throughout } \\
\text { door opening trial } \\
\text { - Most vials showed no } \\
\text { significant temperature } \\
\text { change in response to door } \\
\text { opening } \\
\text { - Only one vial, which was } \\
\text { kept in an open tray rather } \\
\text { than contained in packaging, } \\
\text { showed small temperature } \\
\text { spikes (less than } 0.5^{\circ} \mathrm{C} \text { ) }\end{array}$ \\
\hline Door left ajar & $\begin{array}{l}\text { - Some vial temperatures quickly } \\
\text { exceeded } 8{ }^{\circ} \mathrm{C} \text { (within } 3 \text { to } 5 \\
\text { minutes after opening door), } \\
\text { while others resisted thermal } \\
\text { excursions for up to one hour, } \\
\text { dependent on vial storage } \\
\text { method and location } \\
\text { - All vials resisted thermal } \\
\text { excursions for a longer period of } \\
\text { time with water bottle ballast in } \\
\text { place }\end{array}$ & $\begin{array}{l}\text { - Most vials remained within } \\
2{ }^{\circ} \mathrm{C} \text { to } 8{ }^{\circ} \mathrm{C} \text { even with door } \\
\text { left ajar for up to } 1 \mathrm{~h}\end{array}$ \\
\hline
\end{tabular}




\begin{tabular}{|c|c|c|}
\hline $\begin{array}{l}\text { Increasing room } \\
\text { temperature }\end{array}$ & $\begin{array}{l}\text { - Complex relationship between } \\
\text { room and refrigerator } \\
\text { temperature, dependent on } \\
\text { factors such as refrigerator load } \\
\text { density, timing of a cooling or } \\
\text { defrost cycle, initial refrigerator } \\
\text { temperature, and the time rate of } \\
\text { change of room temperature } \\
\text { - To cause internal refrigerator } \\
\text { temperature to change by } 1^{\circ} \mathrm{C} \text {, } \\
\text { ambient temperature must vary } \\
\text { by }>20^{\circ} \mathrm{C} \\
\text { - Overall effect of room } \\
\text { temperature fluctuation is small } \\
\text { and unlikely to impact } \\
\text { refrigerator performance } \\
\text { - Continuous temperature } \\
\text { monitoring system should be } \\
\text { used to identify and mitigate any } \\
\text { possible risks associated with } \\
\text { large changes in ambient } \\
\text { conditions }\end{array}$ & $\begin{array}{l}\text { - Room temperature changes } \\
\text { have very little impact on } \\
\text { refrigerator's ability to } \\
\text { maintain internal temperature } \\
\text { stability } \\
\text { - To cause internal refrigerator } \\
\text { temperature to change by } \\
1^{\circ} \mathrm{C} \text {, ambient temperature } \\
\text { must vary by }>50^{\circ} \mathrm{C}\end{array}$ \\
\hline Power outage & $\begin{array}{l}\text { - Depending on vial location and } \\
\text { storage method, and it takes } \\
1 \mathrm{~h} \text { to } 4 \mathrm{~h} \text { for vials to exceed } \\
8{ }^{\circ} \mathrm{C} \text { after power off } \\
\text { - Water bottle ballast provided an } \\
\text { extra } 32 \text { min to } 2 \mathrm{~h} 37 \mathrm{~min} \\
\text { before vaccines exceeded limit } \\
\text { - Vials stored in boxes or original } \\
\text { packaging, stored away from the } \\
\text { upper region of refrigerator had } \\
\text { slowest temperature increase } \\
\text { - Requires } 9 \mathrm{~h} \text { to } 12 \mathrm{~h} \text { to fully re- } \\
\text { equilibrate following an outage }\end{array}$ & $\begin{array}{l}\text { - Depending on vial location } \\
\text { and storage method, and it } \\
\text { takes } 45 \text { min to } 2 \mathrm{~h} 19 \mathrm{~min} \\
\text { for vials to exceed } 8{ }^{\circ} \mathrm{C} \text { after } \\
\text { power off } \\
\text { - Glass sliding doors do not } \\
\text { provide as much thermal } \\
\text { insulation as traditional } \\
\text { refrigerator doors } \\
\text { - Power outages pose a greater } \\
\text { risk to vaccines stored in } \\
\text { refrigerators with glass doors, } \\
\text { as they may be exposed to } \\
\text { out-of-range temperatures } \\
\text { much sooner }\end{array}$ \\
\hline Defrost cycle & $\begin{array}{l}\text { - Defrost cycle runs every } 28 \mathrm{~h} \text { to } \\
34 \mathrm{~h} \\
\text { - Most vials remained within } 2{ }^{\circ} \mathrm{C} \\
\text { to } 8{ }^{\circ} \mathrm{C} \text { during defrost cycle, one } \\
\text { vial exceeded } 8{ }^{\circ} \mathrm{C} \text { for } \sim 10 \text { min } \\
\text { - Thermometers placed in air / } \\
\text { near walls recorded dramatic } \\
\text { temperature spike followed by a } \\
\text { drop below } 2{ }^{\circ} \mathrm{C}\end{array}$ & $\begin{array}{l}\text { - Automated defrost cycle } \\
\text { - No discernible effect on } \\
\text { refrigerator chamber } \\
\text { temperature }\end{array}$ \\
\hline
\end{tabular}




\begin{tabular}{|c|c|c|}
\hline $\begin{array}{l}\text { General stability of vial } \\
\text { temperature }\end{array}$ & $\begin{array}{l}\text { - Good } \\
\text { - Vaccine vials remained within } \\
\text { specified temperature range } \\
\text { during regular operation, } \\
\text { regardless of packing density or } \\
\text { type }\end{array}$ & $\begin{array}{l}\text { - Excellent } \\
\text { - Vaccine vials remained } \\
\text { within specified temperature } \\
\text { range during regular } \\
\text { operation, regardless of } \\
\text { packing density or type, as } \\
\text { well as during door opening } \\
\text { trials } \\
\text { - Average vial temperatures } \\
\text { varied by less than } 1.5^{\circ} \mathrm{C} \\
\text { throughout entire range of } \\
\text { testing }\end{array}$ \\
\hline
\end{tabular}




\section{ACKNOWLEDGMENTS}

We thank the Centers for Disease Control and Prevention for providing financial support for this project. Additional thanks to Dawn Cross, who assisted with the Vaccines for Children clinic tours.

\section{REFERENCES}

1. Chojnacky, M. J.; Miller, W. W.; Ripple, D. C.; and Strouse, G. F., "Thermal Analysis of Refrigeration Systems Used for Vaccine Storage”, NISTIR 7656, 2009.

2. Day, G., Rossetto, B., and Ho, J., Testing of Purpose-built Refrigerating Vaccine Storage Cabinets, Test Research Report E175VC-01, Marrickville, New South Wales, 2009.

3. Page, S. L., Earnest, A., Birden, H., Deaker, R., and Clark, C., "Improving vaccination cold chain in the general practice setting," in Australian Family Physician 37, No. 10 (2008).

4. Proceedings of the National Vaccine Storage Workshop, edited by A. Langley, and S. Grant., Queensland Health, Brisbane, 2004.

5. "Performance characteristics of domestic fridges for vaccine storage," E2E IT Solutions Pty. Ltd. Available from: http://www.temptrack.com.au/documents/Fridge Performance Report (Rev A).pdf

6. Vaccine Storage and Handling Toolkit, Centers for Disease Control. Available from: http://www2a.cdc.gov/vaccines/ed/shtoolkit/ 